\title{
Endomorphisms of Smooth Projective 3-Folds with Non-Negative Kodaira Dimension
}

\author{
By \\ Yoshio Fujimoto*
}

\begin{abstract}
Let $X$ be a nonsingular projective 3 -fold with non-negative Kodaira dimension $\kappa(X) \geq 0$ which admits a nonisomorphic surjective morphism $f: X \rightarrow X$ onto itself. If $\kappa(X)=0$ or 2 , a suitable finite étale covering $\tilde{X}$ of $X$ is isomorphic to an abelian 3 -fold or the direct product $E \times S$ of an elliptic curve $E$ and a nonsingular algebraic surface $S$ with $\kappa(S)=\kappa(X)$.
\end{abstract}

\section{$\S 1 . \quad$ Introduction}

The main purpose of this paper is to study the structure of a nonsingular projective 3 -fold $X$ with a surjective morphism $f: X \rightarrow X$ onto itself which is not an isomorphism. We call it a nontrivial surjective endomorphism of $X$.

Let $f: X \rightarrow X$ be a surjective morphism from a nonsingular projective variety $X$ onto itself. Then $f$ is a finite morphism and if the Kodaira dimension $\kappa(X)$ of $X$ is non-negative, $f$ is a finite étale covering. Moreover, if $X$ is of general type or a compact hyperbolic manifold in the sense of Kobayashi [Kob], then $X$ admits no nontrivial surjective endomorphisms (cf. [I], [Kob]). Surjective endomorphisms of a projective manifold with negative Kodaira dimension have been studied by many algebraic geometers (cf. [CS], [D], [HM], [PS]) from the viewpoint of Lazarsfeld's conjecture (cf. [PS], Problem 1). Notably, in [HM, Main Theorem] and [PS, Proposition 2], it has been proved that a rational homogeneous manifold of Picard number 1 has no nontrivial surjective endomorphisms unless it is isomorphic to the complex projective space $\mathbf{P}^{n}$.

Communicated by Y. Miyaoka. Received November 22, 2000. Revised February 19, 2001. 2000 Mathematics Subject Classification(s): 14J15, 14J30, 14D06, 14E30, 32J17.

* Department of Mathematics, Faculty of Education, Gifu University, Gifu 501-11, Japan. e-mail: fujimoto@cc.gifu-u.ac.jp 
The structure of an algebraic surface $S$ which admits a nontrivial surjective endomorphism is fairly simple (cf. Theorem 3.2). If $\kappa(S) \geq 0, S$ is minimal and a suitable finite étale covering of $S$ is isomorphic to an abelian surface or the direct product of an elliptic curve and a smooth curve of genus $\geq 2$. On the other hand, the case of $\kappa(S)=-\infty$ has been studied by Nakayama [N6]: if $S$ is a rational surface, it is a toric surface. And if $S$ is an irrational ruled surface with $q(S) \geq 2$, it is relatively minimal and a suitable finite étale covering $\tilde{S}$ of $S$ is isomorphic to the direct product of $\mathbf{P}^{1}$ and a smooth curve of genus $\geq 2$.

In this paper, we are mainly concerned with the case where $X$ is a smooth projective 3 -fold with non-negative Kodaira dimension. We pose the following question.

Question $\left(E_{n, a}\right)$. Let $X$ be a smooth projective $n$-fold with $0 \leq \kappa(X)=$ $a<n$ which admits a nontrivial surjective endomorphism $f: X \rightarrow X$. Is it true that a suitable finite étale covering $\tilde{X}$ of $X$ has the structure of a smooth abelian scheme over a nonsingular projective variety $W$ with $0 \leq \operatorname{dim}(W)<\operatorname{dim}(X)$ ?

The main theorem of this paper is as follows.

MAIN THEOREM (B). Question $\left(E_{n, a}\right)$ is affirmative in the case where $(n, a)=(3,0)$ or $(3,2)$. Moreover, $\tilde{X}$ can be chosen to be isomorphic to an abelian 3-fold or the direct product $E \times W$ of an elliptic curve $E$ and a smooth projective surface $W$ with $\kappa(W)=\kappa(X)$.

We cannot drop the assumption that a self-map $f: X \rightarrow X$ is a morphism. There are infinitely many examples which admit a generically finite self-rational map $f: X \cdots \rightarrow X$ of degree $\geq 2$, for example, a Kummer surface or a relatively minimal elliptic surface with a global section.

The outline of the proof is as follows. Let $f: X \rightarrow X$ be a nontrivial surjective endomorphism on a smooth projective 3-fold with $\kappa(X) \geq 0$. If the canonical bundle $K_{X}$ of $X$ is not nef, we shall apply the minimal model program (MMP) (cf. [Mo1], [Mo2], [KM]) to the morphism $f: X \rightarrow X$. Contrary to the case of algebraic surfaces, $X$ is not necessarily minimal in the sense of [Mo2], [KM], but each extremal ray $R$ of $\overline{N E}(X)$ is of type (E1) (cf. [KM]), that is, the contraction morphism $\operatorname{Cont}_{R}: X \rightarrow X^{\prime}$, associated to $R$, is a birational divisorial contraction, which is (the inverse of) the blow-up along a smooth elliptic curve $E$ on $X^{\prime}$. This is a direct consequence of Mori's cone theorem [Mo1], $[\mathrm{KM}]$ and a classification of extremal rays on smooth projective 3 -folds [Mo1]. It follows that there are only finitely many extremal rays of $\overline{N E}(X)$ and $f$ induces a permutation of them. Hence a suitable power $f^{k}(k>0)$ of $f$ has 
the property that $\left(f^{k}\right)_{*} R=R$ for each extremal ray $R$. From the beginning, we may assume that $f_{*} R=R$. The contraction morphism Cont $_{R}: X \rightarrow X^{\prime}$ induces a nontrivial surjective endomorphism $f^{\prime}$ on the nonsingular projective 3 -fold $X^{\prime}$. Note that an elliptic curve $E$ on $X^{\prime}$ which is the center of the blowing-up $\operatorname{Cont}_{R}: X \rightarrow X^{\prime}$ must satisfy $f^{\prime-1}(E)=E$, which is irreducible. By iterating this process finitely many times, we eventually obtain a nontrivial surjective endomorphism $g$ on a nonsingular minimal model $Y:=X_{\min }$ of $X$, which we call a minimal reduction of $f: X \rightarrow X$.

By the abundance theorem by Miyaoka [Mi1], [Mi2] and Kawamata [Kaw2], $K_{Y}$ is semi-ample and a minimal reduction $g: Y \rightarrow Y$ is compatible with the Iitaka fibration of $Y$. Furthermore if $\kappa(X)=0,2$, or $\kappa(X)=1$ and the general fiber of the Iitaka fibration of $Y:=X_{\min }$ is a hyperelliptic surface, we can describe their structure completely, thanks to the Bogomolov's decomposition theorem [Be], the standard fibration theorem by Nakayama [N1] and Fujiki's generic quotient theorem [F2], [F3].

Let us explain briefly the case where $\kappa(X)=2$. The Iitaka fibration of $Y:=X_{\min }$ gives the unique structure of an elliptic fibration $\phi: Y \rightarrow S$ onto a normal surface $S$ with at most quotient singularities. Then by [N1], if we perform a finite succession of flops to $Y$ and take a suitable birational model $T \rightarrow S$, we have an equidimensional elliptic fibration $Y_{1} \rightarrow T$ over $T$. The minimal reduction $g: Y \rightarrow Y$ induces an infinite tower of nonisomorphic finite étale coverings $Y_{1} \leftarrow Y_{2} \leftarrow \cdots \leftarrow Y_{n} \leftarrow \cdots$ between minimal smooth projective 3-folds $Y_{n}$ 's, where $\kappa\left(Y_{n}\right) \geq 0$ and $Y_{n}$ is a minimal model of $X$ for all $n$. Hence they are all isomorphic in codimension one and connected by a finite sequence of flops by Kawamata [Kaw1] and Kollár [Kol]. Hence, if the elliptic fibration $Y_{1} \rightarrow T$ has singular fibers along a codimension one discriminant locus $D$ of $T$, then they must be multiple singular fibers of type ${ }_{m} \mathrm{I}_{0}(m>1)$ whose supports are smooth elliptic curves. Then by [N3], we can show that $Y$ and $Y_{n}$ are all isomorphic and $\phi: Y \rightarrow T$ is a Seifert elliptic fiber space over $T$. In particular, there are no rational curves in fibers of $\phi$. And a suitable finite étale covering $\tilde{Y}$ of $Y$ is isomorphic to the direct product $\tilde{T} \times E$ of an elliptic curve $E$ and a nonsingular minimal algebraic surface $\tilde{T}$ of general type.

Finally, we have to blow-up $Y:=X_{\min }$ successively along smooth elliptic curves, so that $g: Y \rightarrow Y$ can be lifted to recover the original endomorphism $f: X \rightarrow X$. Note that the center of the blowing-up at each step must satisfy very severe conditions and thus we obtain MAIN THEOREM.

The construction of our paper is as follows: In Section 2, we shall give basic facts on the endomorphisms of smooth projective varieties. In Section 3, 
we shall prove the structure theorem of algebraic surfaces with non-negative Kodaira dimension which admit nontrivial surjective endomorphisms. In Section 4, we shall apply the minimal model program (MMP) to a nontrivial surjective endomorphism $f: X \rightarrow X$ on a smooth projective 3 -fold $X$ with $\kappa(X) \geq 0$ and obtain its minimal reduction. As another application of Mori's cone theorem, we shall prove that a surjective morphism $f: S \rightarrow T$ between rational elliptic surfaces $S$ and $T$ is necessarily an isomorphism. The facts from Theorem 4.10 to Corollary 4.14 will not be used to prove MAIN THEOREM. In Section 5, we shall classify the structure of a minimal reduction of $f: X \rightarrow X$ and in Section 6, we shall prove MAIN THEOREM. In Section 7 , as a slight generalization of Theorem 5.1, we shall study the structure of nonsingular projective 3 -folds with $\kappa=2$ which admit an infinite descending sequence of nonisomorphic finite étale coverings.

Notations. In this paper, by a smooth projective $n$-fold $X$, we mean a nonsingular projective manifold of dimension $n$ defined over the complex number field $\mathbf{C}$.

$K_{X}$ : the canonical bundle of $X$.

$\kappa(X)$ : the Kodaira dimension of $X$.

$\chi\left(\mathscr{O}_{X}\right)$ : the Euler-Poincare characteristic of the structure sheaf $\mathscr{O}_{X}$.

$b_{i}(X)$ : the $i$-th Betti number of $X$.

$\operatorname{Div}(X)$ : the group of Cartier divisors on $X$.

$N_{1}(X):=(\{1$-cycles on $X\} / \equiv) \underset{Z}{\otimes} \mathbf{R}$,

$N^{1}(X):=(\{$ Cartier divisors on $X\} / \equiv) \underset{Z}{\otimes} \mathbf{R}$, where $\equiv$ means a numerical equivalence.

$\mathrm{NE}(X):=$ the smallest convex cone in $N_{1}(X)$ containing all effective 1cycles.

$\overline{N E}(X):=$ Kleiman-Mori cone of $X$, i.e. the closure of $\mathrm{NE}(X)$ in $N_{1}(X)$ for the metric topology.

$\rho(X):=\operatorname{dim}_{R} N_{1}(X)$, the Picard number of $X$.

The numerical equivalence class of a 1-cycle $C$ is denoted by $[C]$.

$\sim_{Q}$ : the $\mathbf{Q}$-linear equivalence of $\mathbf{Q}$-divisors on $X$.

$\underset{\text { bir }}{\sim}$ : the birational equivalence of varieties.

$g(C)$ : the genus of a smooth curve $C$.

A complex variety is a reduced and irreducible complex space.

A proper surjective morphism $f: X \rightarrow S$ between complex varieties is called a fibration or a fiber space if $X$ and $S$ are normal and all the fibers of $f$ are connected. 
Elliptic fibration: a fibration $f: X \rightarrow S$ is called an elliptic fibration or an elliptic fiber space if the general fibers are nonsingular elliptic curves. The closed subset $\Delta:=\left\{s \in S, f\right.$ is not smooth at some $\left.x \in f^{-1}(s)\right\}$ is called a discriminant locus of $f$.

A $\mathbf{P}^{1}$-fiber space is a fiber space with general fiber isomorphic to the complex projective line $\mathbf{P}^{1}$.

Let $g: X \cdots \rightarrow Y$ be a meromorphic map of complex varieties, $\Gamma \subseteq X \times Y$ the graph of $g$, and $q: \Gamma \rightarrow Y$ the natural projection. Then we say that $g$ is surjective if $q(\Gamma)=Y . g$ is called a meromorphic fiber space if $q$ is a fiber space.

Let $X$ be a compact complex variety. Then:

$\operatorname{Aut}(X)$ : the complex Lie group of biholomorphic automorphisms of $X$.

$\operatorname{Aut}^{0}(X)$ : the identity component of $\operatorname{Aut}(X)$.

$\operatorname{End}(X)$ : the space of all holomorphic maps from $X$ to itself, which carries a complex structure (cf. $[\mathrm{H}])$.

$\operatorname{Sur}(X)$ : the space of all surjective holomorphic maps $X \rightarrow X$, which is a complex subspace of $\operatorname{End}(X)(\mathrm{cf} .[\mathrm{H}])$.

For $f \in \operatorname{End}(X), f^{k}:=f \circ \cdots \circ f$ stands for the $k$-times composite of $f$.

\section{$\S 2 . \quad$ Basic Facts on Endomorphisms}

Definition 2.1. $\quad$ Let $f: X \rightarrow X$ be a holomorphic map from a normal compact complex space $X$ to itself. We call it an endomorphism of $X$.

Definition 2.2. An endomorphism $f: X \rightarrow X$ of a smooth projective $n$-fold is nontrivial if $f$ is neither a constant map nor an isomorphism.

Lemma 2.3. Let $f: Y \rightarrow X$ be a surjective morphism from a smooth projective $n$-fold $Y$ to a smooth projective $n$-fold $X$. Assume that $\rho(X)=\rho(Y)$. Then

(1) $f$ is a finite morphism. In particular, every surjective endomorphism $g: X \rightarrow X$ of a smooth projective $n$-fold $X$ is a finite morphism.

(2) If $f: X \rightarrow X$ is a surjective endomorphism of a smooth projective $n$-fold with $\kappa(X) \geq 0, f$ is a finite étale covering.

(3) If $f: Y \rightarrow X$ is a finite étale covering, we have $\chi\left(\mathscr{O}_{Y}\right)=\operatorname{deg}(f)$. $\chi\left(\mathscr{O}_{X}\right)$.

Proof. (1) Via the intersection pairing ( , ) of 1-cycles and Cartier divisors, $N_{1}(X)$ and $N^{1}(X)$ are dual to each other and they are real vector spaces of a finite dimension $\rho(X)$. Since $f^{*}: N^{1}(X) \rightarrow N^{1}(Y)$ is injective and $\rho(X)=$ 
$\rho(Y)$ by hypothesis, $f^{*}$ is an isomorphism. Assume that $f$ is not finite. Then there exists an irreducible reduced curve $C$ on $Y$ contracted to a point by $f$. For an ample divisor $L$ of $Y$, we have $L=f^{*}(D)$ for some $D \in N^{1}(X)$. By the projection formula, we have $(C, L)=\left(f_{*} C, D\right)=0$. This contradicts the ampleness of $L$.

(2) By (1), $f$ is a finite morphism. Hence we have $K_{X} \sim f^{*} K_{X}+R$, where $R$ is the ramification divisor of $f$. Hence we have

$$
K_{X} \sim\left(f^{k}\right)^{*} K_{X}+\left(f^{k-1}\right)^{*} R+\cdots+f^{*} R+R \text { for all } k>0,
$$

where $f^{k}$ stands for the $k$-times composite of $f$. Since $\kappa(X) \geq 0, K_{X}$ can be considered to be an effective $\mathbf{Q}$-divisor. Assume that $R \neq 0$. Take an ample divisor $H$ of $X$ and if we let $k \rightarrow \infty$, we have $\left(K_{X}, H^{n-1}\right)=\infty$, which is a contradiction. Hence $R=0$ and the claim follows.

(3) Since $T_{Y}=f^{*} T_{X}$, this follows from the Grothendieck-HirzebruchRiemann-Roch theorem.

Remark. (2) holds true under the assumption that $X$ is a compact complex manifold (cf. [Kob]. Theorem 7.6.11). The proof is essentially the same as above.

Corollary 2.4. (1) If a nontrivial surjective endomorphism $g: X \rightarrow X$ of a smooth projective $n$-fold is a finite étale covering, we have $\chi\left(\mathscr{O}_{X}\right)=0$.

(2) If $g: X \rightarrow X$ is a nontrivial surjective endomorphism of a smooth projective $n$-fold with non-negative Kodaira dimension, we have $\chi\left(\mathscr{O}_{X}\right)=0$.

Proof. From Lemma 2.3, we have $\chi\left(\mathscr{O}_{X}\right)=\operatorname{deg}(g) \cdot \chi\left(\mathscr{O}_{X}\right)$. Since $\operatorname{deg}(g)>$ 1 , the claim follows. (2) is derived from (1) and Lemma 2.3.

Next, we show that a surjective endomorphism $f: X \rightarrow X$ is compatible with the Iitaka fibration and the Albanese map of $X$.

Proposition 2.5. Let $\phi_{\left|m K_{X}\right|}: X \cdots \rightarrow Y \subset \mathbf{P}^{n}$ be the Iitaka fibration of a smooth projective $n$-fold $X$ with $\kappa(X) \geq 0$. Assume that $X$ admits a nontrivial surjective endomorphism $f: X \rightarrow X$. Then there exists a biregular automorphism $h$ of $Y$ such that $\phi \circ f=h \circ \phi$.

Proof. Since $f$ is a finite étale covering and $K_{X} \sim f^{*} K_{X}, f$ induces an automorphism $f^{*}$ of $H^{0}\left(X, \mathscr{O}\left(m K_{X}\right)\right)$. Hence $f$ also induces an automorphism $h$ of $Y$ with the required property.

The following proposition is well known (cf. [I], [Kob]). Here we shall give an elementary proof. 
Proposition 2.6. Let $f: X \rightarrow X$ be a surjective endomorphism of a smooth $n$-fold $X$ of general type. Then $f$ is an isomorphism.

Proof. By assumption, the Iitaka fibration $\phi: X \cdots \rightarrow Y$ gives a birational map. Hence by Proposition 2.5, $f$ is also a birational map. Moreover, $f$ is a finite étale covering by Lemma 2.3 , hence is an isomorphism.

Proposition 2.7. Let $f: X \rightarrow X$ be a surjective endomorphism of a smooth projective $n$-fold and $X \stackrel{g}{\longrightarrow} Z \stackrel{h}{\longrightarrow} \alpha(X) \subset \mathrm{Alb}(X)$ the Stein factorization of the Albanese map $\alpha: X \rightarrow \operatorname{Alb}(X)$ of $X$. Then there exists a surjective endomorphism $\mu$ of $Z$ such that $g \circ f=\mu \circ g$.

Proof. From the universality of the Albanese map, there exists a unique affine (in the sense of the composite of translations and endomorphisms of abelian varieties) morphism $\mu: \operatorname{Alb}(X) \rightarrow \operatorname{Alb}(X)$ such that $\alpha \circ f=\mu \circ \alpha$. Hence, for any $p \in Z, g^{-1}(p)$ is mapped to a point on $Z$ by $g \circ f$, since $g^{-1}(p)$ is connected. Hence there exists a surjective endomorphism $\mu$ of $Z$ with the required property.

We give some properties of the action of biregular automorphism groups on an endomorphism.

Proposition 2.8. Let $f: X \rightarrow X$ be a surjective endomorphism of a smooth projective $n$-fold with $\kappa(X) \geq 0$. Assume that $T:=\operatorname{Aut}^{0}(X)$ is a positive dimensional abelian variety. Then there exists a natural complex structure on the quotient space $Y:=X / T$ such that the projection $p: X \rightarrow Y$ is a Seifert abelian fiber space over $Y$ (i.e. $p$ has at most multiple singular fibers and is a principal fiber bundle with typical fiber and structure group $T$ outside them). Moreover, $f$ induces a surjective endomorphism $u$ of $Y$ such that $p \circ f=u \circ p$.

Lemma 2.9 (cf. $[\mathrm{H}])$. Let $f: X \rightarrow X$ be a surjective endomorphism of a normal compact complex space $X$ with $\kappa(X) \geq 0$. Assume that $X$ is in the class $\mathscr{C}$ in the sense of Fujiki $[\mathrm{F} 1]$, [F3]. Then for all $g \in \operatorname{Aut}^{0}(X)$, there exists a unique $h \in \operatorname{Aut}^{0}(X)$ such that $f \circ g=h \circ f$.

Proof. By Fujiki $[\mathrm{F} 1], T:=\operatorname{Aut}^{0}(X)$ is a complex torus. In particular, it is compact. The right $T$-orbit of $f, f \circ T:=\{f \circ g \mid g \in T\}$, is a compact subvariety of $\operatorname{Sur}(X)$. Hence by Horst $[\mathrm{H}]$, Theorem 3.1, it is contained in a left $T$-orbit of $f$, that is, $f \circ T \subset T \circ f$. Since $f$ is a finite morphism, the morphism $T \rightarrow f \circ T$ has finite fibers. Hence we have $\operatorname{dim} T=\operatorname{dim} f \circ T \leq \operatorname{dim} T \circ f=$ 
$\operatorname{dim} T$. Since $T \circ f \cong T$ is irreducible, we have $f \circ T=T \circ f$ and the claim follows. The uniqueness of $h$ follows from the surjectivity of $f$.

Proof of Proposition 2.8. By Holman's theorem (cf. [F1] p. 248, 5.1), there exists a natural complex structure on the orbit space $Y=X / T$ such that the projection $p: X \rightarrow Y$ is a Seifert abelian fiber space over $Y$. Since $f \circ T=T \circ f$, $f$ induces an endomorphism $u$ of $Y$. Since $p$ is equidimensional and $f$ is finite, $u$ is also finite by an easy dimension count.

Proposition 2.10. Let $X$ be a smooth projective variety with $\kappa(X) \geq 0$. Assume that $T:=\operatorname{Aut}^{0}(X)$ is a positive dimensional abelian variety and there exists a nontrivial surjective endomorphism $f: X \rightarrow X$ of $X$.

Then we have the following commutative diagram such that

(1) The Iitaka fibration $\phi: X \cdots \rightarrow Z$ factors through the Seifert abelian fiber space $p: X \rightarrow Y:=X / T$ and induces a meromorphic fiber space $g$ : $Y \cdots \rightarrow Z$,

(2) $f$ induces a surjective endomorphism $u$ of $Y$ and an automorphism $h$ of $Z$,

(3) Let $Y_{z}(z \in Z)$ be a general fiber of $g: Y \cdots \rightarrow Z$. Then we have $\kappa\left(Y_{z}\right) \leq 0$ and the Albanese map $\alpha_{z}: Y_{z} \rightarrow \operatorname{Alb}\left(Y_{z}\right)$ gives $Y_{z}$ a fiber space structure over $\operatorname{Alb}\left(Y_{z}\right)$.

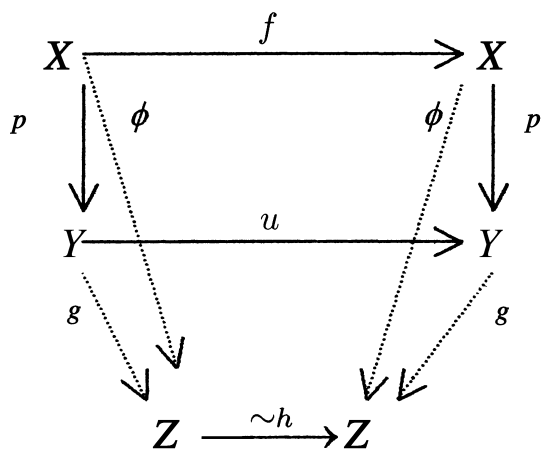

Proof. (1) Let $m$ be a positive integer such that $P_{m}(X)>0$. Then $T:=$ $\operatorname{Aut}^{0}(X)$ acts on $H^{0}\left(X, \mathscr{O}\left(m K_{X}\right)\right)$ and induces a holomorphic representation $\varphi: T \rightarrow \operatorname{GL}\left(H^{0}\left(X, \mathscr{O}\left(m K_{X}\right)\right)\right.$. Since $T$ is complete and GL is affine, $\varphi$ is a trivial representation. Hence the general fiber of $p$ is mapped to a point by $\phi$ and the claim follows. (2) is derived from Proposition 2.8. 
(3) For general $z \in Z, P_{z}:=\left.P\right|_{X_{z}}: X_{z} \rightarrow Y_{z}$ is a Seifert abelian fiber space. Hence by the addition theorem (cf. [U]), we have: $\kappa\left(Y_{Z}\right) \leq \kappa\left(X_{Z}\right)=0$. From the universality of the Albanese map, there exists an affine morphism $u: \operatorname{Alb}\left(X_{z}\right) \rightarrow \operatorname{Alb}\left(Y_{z}\right)$ such that $u \circ \alpha_{X_{z}}=\alpha_{Y_{z}} \circ P_{z}$. By Fujiki [F1, Lemma 5.2 and Proposition 5.4], we have $q\left(X_{z}\right)=q\left(Z_{z}\right)+\operatorname{dim}(T)$ and the Albanese map $\alpha_{X_{z}}: X_{z} \rightarrow \operatorname{Alb}\left(X_{z}\right)$, when restricted to each fiber of $P_{z}$, gives a finite morphism. It is surjective, since the image of $\alpha_{X_{z}}$ generates $\operatorname{Alb}\left(X_{z}\right)$. Hence each fiber of $u$ is connected and the Albanese map of $Y_{z}$ gives a fiber space structure if the Albanese map of $X_{z}$ gives a fiber space structure. Since $\kappa\left(X_{z}\right)=$ 0, Kawamata's theorem [Kaw3] implies the claim.

As an application of Lemma 2.3 and Proposition 2.7, we show the following proposition.

Proposition 2.11. Let $C$ be a hyperelliptic curve of genus $g \geq 2$, where $g$ is an even integer. Then $S^{g} C(:=$ the $g$-th symmetric product of $C)$ has no nontrivial surjective endomorphism.

Proof. Let $f: S^{g} C \rightarrow S^{g} C$ be a surjective endomorphism of $S^{g} C$. It is well known that the Albanese map $\alpha: S^{g} C \rightarrow \operatorname{Jac}(C)$ gives a birational morphism. From the universality of the Albanese map, $f$ induces a surjective endomorphism $g$ of $\operatorname{Jac}(C)$ such that $\alpha \circ f=g \circ \alpha$. Since $C$ is a hyperelliptic curve of genus $g=2 k(k \in N), W_{g}^{k}(C)$ consists of a unique element $L:=\left|\sum_{j=1}^{k}\left[Q_{j}+i\left(Q_{j}\right)\right]\right|$, where $Q_{j} \in C, 1 \leq j \leq k$ and $i: C \rightarrow C$ is a hyperelliptic involution of $C$ (cf. $[\mathrm{Mu}]$ ). By $\alpha, L \cong \mathbf{P}^{k}$ is contracted to a point $O \in \operatorname{Jac}(C)$, which is the zero element in the group law of $\operatorname{Jac}(C)$. Since $\kappa\left(S^{g} C\right)=\kappa(\operatorname{Jac}(C))=0, f$ and $g$ are finite étale coverings by Lemma 2.3. Since $L \cong \mathbf{P}^{k}$ is simply connected, $f^{-1}(L)=\alpha^{-1}\left(g^{-1}(O)\right)$ consists of $d(:=\operatorname{deg}(f))$ disjoint unions of $\mathbf{P}^{k}$ s. For an irreducible subvariety $M$ of $S^{g} C$, we note that:

(i) $\operatorname{dim} M \leq k$ if $\alpha(M)$ is a point, and

(ii) $M=L$ if furthermore $\operatorname{dim} M=k$.

Hence we have $\operatorname{deg}(g)=1$ and $g$ is an isomorphism. Since $\alpha$ is birational, $f$ is also a birational morphism, hence is an isomorphism.

The following results will be used to prove MAIN THEOREM in the case of $\kappa(X)=0$.

Proposition 2.12. $\quad$ Let $X$ be a compact complex manifold in the class $\mathscr{C}$ in the sense of Fujiki [F2] (i.e. bimeromorphic to a compact complex Kähler 
manifold) and $A$ an abelian variety. If $b_{1}(X)=0$, then for any surjective endomorphism $f$ of $X \times A$, there exists a surjective endomorphism $g$ (resp. $h$ ) of $X$ (resp. A) such that $f=g \times h: X \times A \rightarrow X \times A$.

Proof. By assumption, the second projection $p: X \times A \rightarrow A$ coincides with the Albanese map of $X \times A$. From the universality of the Albanese map (or by Proposition 2.7), $f$ induces a surjective endomorphism $h$ of $A$ such that $p \circ f=h \circ p$. Hence, for each $a \in A$, if we denote by $f_{a}$ the restriction of $f$ to $p^{-1}(a) \cong X$, we obtain a holomorphic map $\varphi: A \rightarrow \operatorname{Sur}(X)$ defined by $\varphi(a)=f_{a}$. Since $\varphi(A)$ is a compact subvariety of $\operatorname{Sur}(X)$, it follows from $\left[\mathrm{H}\right.$, Theorem 3.1] that $\varphi(A)$ is contained in a left $\operatorname{Aut}(X)$-orbit of $g:=f_{0}$, where $0 \in A$ is the identity element. Therefore, for each $a \in A$, there exists a unique $j_{a} \in \operatorname{Aut}(X)$ such that $f_{a}=j_{a} \circ g$, because $g$ is surjective. Thus we obtain a holomorphic map $u: A \rightarrow \operatorname{Aut}(X)$ defined by $u(a)=j_{a}, a \in A$. On the other hand, since $X \in \mathscr{C}$ and $b_{1}(X)=0$, Aut $(X)$ has the structure of a linear algebraic group by [F1, Corollary 5.8]. Hence $u$ is a constant map. Since $u_{0}=i d_{X}$, we have $u_{a} \equiv i d_{X}$ and $f_{a} \equiv g$ for all $a \in A$. Hence we have $f=g \times h: X \times A \rightarrow X \times A$.

Corollary 2.13. Let $S$ be a $K 3$ surface and $E$ an elliptic curve. For any surjective endomorphism $f$ of $S \times E$, there exists an automorphism $g$ of $S$ and an affine morphism $h: E \rightarrow E$ such that $f=g \times h: S \times E \rightarrow S \times E$.

Proof. The claim immediately follows from Proposition 2.12 and Theorem 3.2 .

Proposition 2.14. $\quad$ Let $X$ be a rationally connected, projective manifold (cf. [KoMiMo]) and $A$ an abelian variety. For any étale surjective endomorphism $f$ of $Z:=X \times A$, there exists an affine morphism $h: A \rightarrow A$ and an automorphism $g$ of $X$ such that

$$
f=g \times h: Z \rightarrow Z \text {. }
$$

Proof. By [KoMiMo], $X$ is simply connected. Hence the claim follows from Proposition 2.12.

Proposition 2.15. $\quad$ Let $X$ (resp. $Y$ ) be a nonsingular projective variety of general type (resp. $\kappa(Y)=0$ ). Then for any surjective endomorphism $f$ of $X \times Y$, there exists a holomorphic family $\left\{\varphi_{x}\right\}_{x \in X}$ of automorphisms of $Y$ parametrized by $X$ such that $\left(i d_{X} \times \varphi_{x}\right) \circ f=g \times h$, where $g$ (resp. $\left.h\right)$ is an automorphism of $X$ (resp. surjective endomorphism of $Y$ ). 
Proof. Let $p: X \times Y \rightarrow X$ be the first projection and $\phi: X \cdots \rightarrow Z$ the Iitaka fibration of $X$. Since $\kappa(Y)=0$, the composite map $\psi:=\phi \circ p$ : $X \times Y \cdots \rightarrow Z$ is birational to the Iitaka fibration of $X \times Y$. By Proposition 2.5 , there exists a biregular automorphism $u$ of $Z$ such that $u \circ \psi=\psi \circ f$. Since $X$ is of general type, $\phi$ is a birational map and $p \circ f \circ p^{-1}(x)$ is a point of $X$ for 'general' $x \in X$, hence for all $x \in X$ by the rigidity lemma (cf. [KM, Lemma 1.6]). Then there exists a surjective endomorphism $g$ of $X$ such that $g \circ p=p \circ f$. Since $X$ is of general type, $g$ is an automorphism of $X$ by Proposition 2.6. Therefore, for each $x \in X$, if we denote by $f_{x}$ the restriction of $f$ to $p^{-1}(x) \cong Y$, we define the morphism $\alpha: X \rightarrow \operatorname{Sur}(Y)$ by $\alpha(x)=f_{x}$. Since $\alpha(X)$ is a compact subvariety of $\operatorname{Sur}(X)$, Horst's theorem $([\mathrm{H}])$ implies that $\alpha(X)$ is contained in a left $\operatorname{Aut}(Y)$-orbit of some $h \in \operatorname{End}(Y)$. Hence for each $x \in X$, there exists a unique $j_{x} \in \operatorname{Aut}(Y)$ which depends holomorphically on $x \in X$ such that $f_{x}=j_{x} \circ h$. If we put $\varphi_{x}:=j_{x}^{-1} \in \operatorname{Aut}(X)$, we obtain the desired claim.

Corollary 2.16. Under the same assumption as in Proposition 2.15, we further assume that $b_{1}(Y)=0$. Then there exist $g \in \operatorname{Aut}(X)$ and $h \in \operatorname{Sur}(Y)$ such that $f=g \times h$.

Proof. Since $\operatorname{Aut}(Y)$ is a linear algebraic group by Fujiki [F1], the morphism $t: X \rightarrow \operatorname{Aut}(Y)$ defined by $t(x):=j_{x}, x \in X$, is a constant map and the claim follows.

Example. Let $X$ be a projective manifold of general type and $A$ an Abelian variety. Every surjective endomorphism $f$ of $X \times A$ can be expressed as $f(x, \varsigma)=(g x, P \varsigma+t(x)),(x, \varsigma) \in X \times A$, where $P: A \rightarrow A$ is a group homomorphism of $A$ and $t: X \rightarrow A$ is a morphism.

\section{$\S 3 . \quad$ Surface Case}

In this section, we shall study the structure of algebraic surfaces with nonnegative Kodaira dimension which admit nontrivial surjective endomorphisms.

Proposition 3.1. $\quad$ Let $S$ be an algebraic surface with $\kappa(S) \geq 0$. Suppose that $S$ admits a nontrivial surjective endomorphism $f: S \rightarrow S$. Then $S$ is minimal.

Proof. Assume that $S$ is not minimal and take a $(-1)$-curve $e$ on $S$. Since $f$ is a finite étale covering and $e\left(\cong \mathbf{P}^{1}\right)$ is simply connected, $f^{-1}(e)$ 
(the set-theoretic inverse image of $e$ ) consists of $d:=\operatorname{deg}(f)(\geq 2)$ disjoint smooth rational curves $C_{i}{ }^{\prime}$ s $(1 \leq i \leq d)$. Since $K_{S} \sim f^{*} K_{S}$, it follows by the projection formula that each $C_{i}$ is a $(-1)$-curve. By iterating this process, there exist infinitely many mutually disjoint $(-1)$-curves on $S$, which contradicts the finiteness of $\rho(S)$.

Remark. $\quad$ Proposition 3.1 is derived from Proposition 4.2. Here we give an elementary proof.

Theorem 3.2. $\quad$ Let $S$ be an algebraic surface with $\kappa(S) \geq 0$ which admits a nontrivial surjective endomorphism. Then $S$ is minimal and one of the following cases occurs.

Case (1). If $\kappa(S)=0, S$ is either a hyperelliptic surface or an abelian surface.

Case (2). If $\kappa(S)=1$, the Iitaka fibration $\phi: S \rightarrow C$ gives the unique structure of a minimal elliptic surface over a curve $C$. It is a Seifert elliptic fibration, that is, it has at most multiple singular fibers of type ${ }_{m} \mathrm{I}_{0}$ in the sense of Kodaira and is a principal fiber bundle outside them. And a suitable finite étale covering $T$ of $S$ is isomorphic to the direct product $\tilde{C} \times E$ of an elliptic curve $E$ and a smooth curve $\tilde{C}$ of genus $g(\tilde{C}) \geq 2$.

Proof. By Proposition 2.6, $S$ is not of general type. First assume that $\kappa(S)=0 . S$ is minimal by Proposition 3.1. By Corollary 2.4, $S$ is neither a $K 3$ surface nor an Enriques surface. Hence by the classification theory of algebraic surfaces, $S$ is either a hyperelliptic surface or an abelian surface.

Next we shall consider the case where $\kappa(S)=1$. Since $S$ is minimal by Proposition 3.1, $K_{S}$ is semi-ample and the Iitaka fibration $\phi: S \rightarrow C$ gives $S$ the unique structure of an elliptic surface. By Corollary 2.4, we have $\chi\left(\mathscr{O}_{S}\right)=0$. Hence the desired result follows from the theory of an elliptic surface due to Kodaira.

Example. Let $C$ be a smooth hyperelliptic curve of genus $g \geq 2$ with the hyperelliptic involution $\iota: C \rightarrow C$. Let $E$ be a smooth elliptic curve and $t$ the translation of order 2 on $E$. Let $j:=(i, t)$ be the involution on $C \times E$. Its action is free and the quotient $S:=C \times E /\langle j\rangle$ is a smooth algebraic surface with $\kappa(S)=1$. The first projection $p: C \times E \rightarrow C$ induces on $S$ an elliptic fiber space structure $f: S \rightarrow C /\langle i\rangle \cong \mathbf{P}^{1}$, which has $(2 g+2)$ multiple fibers of type ${ }_{2} \mathrm{I}_{0}$ and is a principal fiber bundle outside them. For any odd integer $n \geq 3$, let $[n]: E \rightarrow E$ be multiplication by $n$. Then $\varphi:=\left(i d_{C},[n]\right): C \times E \rightarrow C \times E$ 
commutes with the action of $j$ and induces a nontrivial surjective endomorphism $g$ of $S$ over $C$.

Remark. (1) In the case where $\kappa(S)=1$, we shall give an alternative proof without using the fact that $\chi\left(\mathscr{O}_{S}\right)=0$, since the same method applies to the proof of MAIN THEOREM in the case of $\kappa(X)=2$.

For a sufficiently large positive integer $m$, we have $m K_{S} \sim \phi^{*} L$ for a very ample line bundle $L$ on $C$. Hence by Proposition 2.5, for every positive integer $k$, there exists an automorphism $h_{k}$ of $C$ such that $\phi \circ f^{k}=h_{k} \circ \phi$. Assume that $\phi: S \rightarrow C$ has a singular fiber $F_{p}$ over a point $p \in C$, which is not of type ${ }_{m} \mathrm{I}_{0}$. Then each irreducible component of $F_{p}$ is a smooth rational curve, or $F_{p}$ is a cuspidal cubic (called type II) or a nodal cubic (called type $\mathrm{I}_{1}$ ). In the first case, if we let $k \rightarrow \infty$, there exist infinitely many $\mathbf{P}^{1}$ 's in a singular fiber of $\phi$, since $\mathbf{P}^{1}$ is simply connected and $f$ is étale. This is a contradiction. In the second and the third cases, if we let $k \rightarrow \infty$, there exist infinitely many cusps or nodes in a singular fiber of $\phi$, which again contradict Kodaira's classification of singular fibers of elliptic surfaces.

(2) We cannot drop the assumption that a self-map $f: X \rightarrow X$ is a morphism. There exist infinitely many examples which admit a generically finite self-rational map $f: X \cdots \rightarrow X$ of degree $\geq 2$.

Example 1. Let $A$ be an abelian surface and $X:=\operatorname{Km}(A)$ the Kummer surface associated to $A$. For any positive integer $n \geq 2$, let $[\mathrm{n}]: A \rightarrow A$ be multiplication by $n$. Then it commutes with the involution $\iota$ of $A$ and induces a self-rational map $g: X \cdots \rightarrow X$ of degree $\geq 2$.

Example 2. Let $f: S \rightarrow C$ be a relatively minimal elliptic surface with a global section $s$. Then $S$ can be considered as an elliptic curve $\mathscr{E}$ over the function field $K$ of $C$ with the origin $s$. For any positive integer $n \geq 2$, let $[\mathrm{n}]: \mathscr{E} \rightarrow \mathscr{E}$ be multiplication by $n$ in the group law of $\mathscr{E}$ over $K$. It induces a generically finite rational map $[\mathrm{n}]: S \cdots \rightarrow S$ of degree $\geq 2$ onto itself. Furthermore, if a rational curve is contained in a singular fiber of $f,[\mathrm{n}]$ is not a morphism by Theorem 3.2 .

Example 3 (cf. [F1, p. 254. Example]). Let $E$ be a smooth elliptic curve and $t$ (resp. $j$ ) the translation of order 2 on $E$ (resp. the group inversion $j(\varsigma)=$ $-\varsigma, \varsigma \in E)$. Let $\iota=t \times j$ be the involution on $E \times E$. Then the quotient $S:=E \times E /\langle\iota\rangle$ is a hyperelliptic surface. The first projection $E \times E \rightarrow E$ induces an elliptic fibration $\pi: S \rightarrow E^{\prime}:=E /\langle t\rangle$, which is a principal fiber bundle with typical fiber $E$ over the elliptic curve $E^{\prime}$ and coincides with the 
Albanese map of $S$. The second projection $E \times E \rightarrow E$ induces a Seifert elliptic fibration $p: S \rightarrow E / j \cong \mathbf{P}^{1}$. (i.e. It has 4 multiple singular fibers of type ${ }_{2} \mathrm{I}_{0}$ and is a principal fiber bundle outside them). We have $\operatorname{Aut}^{0}(S) \cong E$ and $p$ coincides with the quotient map $S \rightarrow S / E$ (cf. Proposition 2.8). Let $f$ be an endomorphism of $E \times E$ defined by $f(x,[\varsigma])=(x,[2 \varsigma])$. It commutes with the action of $\iota$ and induces an endomorphism $g$ of $S$ which are compatible with the elliptic fiber space structures $p: S \rightarrow E^{\prime}$ and $q: S \rightarrow \mathbf{P}^{1}$ (cf. Proposition 2.8). In a similar fashion, we can show that every hyperelliptic surface has a nontrivial surjective endomorphism.

A converse statement to Theorem 3.2 also holds:

Proposition 3.3. Every minimal algebraic surface with $\kappa(S)=1$ and $\chi\left(\mathscr{O}_{S}\right)=0$ admits a nontrivial surjective endomorphism.

Proof. This can be checked directly by Kodaira's theory of elliptic surfaces. Here we shall give a coordinate free description by using Nakayama's theory of $\partial$-étale cohomology. The Iitaka fibration $f: S \rightarrow C$ gives $S$ the unique structure of a Seifert elliptic surface. $f$ has multiple fibers of type ${ }_{m} \mathrm{I}_{0}$ over finite points $D(\subset C)$ and is a principal fiber bundle outside them. Let $H:=\left.\left(R^{1} f_{*} \mathbf{Z}_{S}\right)\right|_{C \backslash D}$ be the local system which has a polarized variation of Hodge structure of rank $=2$ and weight $=1$. Since local monodromies around $D$ are trivial, $H$ can be extended to the whole $C$ as a variation of Hodge structure. By [N2], $f: S \rightarrow C$ is considered as a torsor in $\partial$-étale topology on the $\partial$-space $\underline{C}=(C, D)$ of the smooth basic elliptic fibration $p: B(H) \rightarrow C$ associated with $H$. Since $S$ is algebraic, it can be expressed as $S=B^{\eta}$, where $\eta \in H^{1}\left(\underline{C}, \mathscr{S}_{H / \underline{C}}\right)$ is of finite order $N$. (Here $\mathscr{S}_{H / \underline{C}}$ is the sheaf of germs of meromorphic sections of $p$ in the $\partial$-étale topology.) Let $d$ be a positive integer with $d \equiv 1(\bmod N)$ and $[\mathrm{d}]: B(H) \rightarrow B(H)$ multiplication by $d$. It induces a nontrivial surjective endomorphism $g: S=B^{\eta} \rightarrow B^{d \eta} \cong B^{\eta}=S$.

Remark. Proposition 3.3 does not necessarily hold if $S$ is nonprojective. We shall give such an example.

Let $L$ be a line bundle on a smooth projective $(n-1)$-fold $V$ of general type and $\boldsymbol{L}$ the total space of $L$. Take an elliptic curve $E \cong \mathbf{C}^{*} /\langle\rho\rangle(\rho \in$ $\left.\mathbf{C}^{*}, 0<|\rho|<1\right)$ without complex multiplication. The cyclic group $\langle\rho\rangle(\cong \mathbf{Z})$ acts on $\boldsymbol{L}^{*}:=\boldsymbol{L} /\{0$-section $\}$ by multiplication fiberwisely. Its action is free and properly discontinuous, hence the quotient space $X:=\boldsymbol{L}^{*} /\langle\rho\rangle$ is an $n$ dimensional compact complex manifold. By the natural projection $p: X \rightarrow V$, $X$ is an elliptic bundle over $V$ with typical fiber and structure group $E$. $X$ is 
Kähler if and only if the first Chern class $c_{1}(L) \in H^{2}(V, \mathbf{Z})$ of $L$ is of finite order (cf. [Fm2]). Moreover, if $q(V)>0$ and $c_{1}(L)$ is of finite order, $X$ is a compact Kähler $n$-fold with algebraic dimension $a(X)=n-1$ for 'general' $L \in \operatorname{Pic}(V)$.

Proposition 3.4. $\quad$ Suppose that $X$ is non-Kähler or Kähler with algebraic dimension $a(X)=n-1$. Then $X$ has no nontrivial surjective endomorphisms.

Proof. Assume that there exists a nontrivial surjective endomorphism $f$ : $X \rightarrow X$. Since $K_{X} \cong p^{*} K_{V}$ and $V$ is of general type, there exists $u \in \operatorname{Aut}(V)$ such that $u \circ p=p \circ f$ by the same argument as in the proof of Proposition 2.14. Since $V$ is of general type, $u$ is of finite order $m>0$. By replacing $f$ by $m$-times power $f^{m}$ of $f$, we may assume that $f \in \operatorname{End}(X)$ induces an identity map on $V . p: X \rightarrow V$ can be considered as a torsor of the trivial elliptic bundle $V \times E \rightarrow V$. It can be expressed as $X=(V \times E)^{\eta}$, where $\eta \in H^{1}(V, \mathscr{O}(E))$ is of infinite order, since $X$ is nonprojective (cf. [N1]). (Here $\mathscr{O}(E)$ denotes the sheaf of germs of holomorphic functions on $V$ with values in the elliptic curve $E$.) After composing a suitable automorphism $u$ of $X$ with $f, u \circ f \in \operatorname{End}(X)$ coincides with multiplication by $d$ for some integer $d>1$, since the elliptic curve $E$ has no complex multiplication. This contradicts the assumption that $\eta$ is of infinite order.

\section{$\S 4$. Minimal Reduction}

In this section, we shall apply the minimal model program (MMP) to the morphism $f: X \rightarrow X$, where $f$ is a nontrivial surjective endomorphism of a smooth projective 3 -fold $X$ with $\kappa(X) \geq 0$.

Let $f: Y \rightarrow X$ be a finite surjective morphism between smooth projective $n$-folds. The norm map $f_{*}: \operatorname{Div}(Y) \rightarrow \operatorname{Div}(X)$ can be extended R-linearly to the push-forward map $f_{*}: N^{1}(Y) \rightarrow N^{1}(X)$. Via the intersection pairing of 1-cycles and Cartier divisors, $N_{1}(X)$ and $N^{1}(X)$ are dual to each other. We define the pull-back map $f^{*}: N_{1}(X) \rightarrow N_{1}(Y)$ to be the adjoint map of $f_{*}$.

We recall the following proposition due to Mori.

Proposition 4.1 (cf. [Mo1, Proposition 1.9]). Let $f: Y \rightarrow X$ be a $f$ nite surjective morphism between smooth projective $n$-folds. Then $f^{*}: N_{1}(X) \rightarrow$ $N_{1}(Y)$ is injective and $f^{*} \overline{N E}(X)=f^{*} N_{1}(X) \cap \overline{N E}(Y)$.

We prove some facts that provide the key to many of the results in this section. 
Proposition 4.2. Let $f: Y \rightarrow X$ be a finite surjective morphism between smooth projective $n$-folds with $\rho(X)=\rho(Y)$.

(1) Then $f^{*}: N_{1}(X) \rightarrow N_{1}(Y)$ (resp. $f_{*}: N_{1}(Y) \rightarrow N_{1}(X)$ ) is an isomorphism and

$$
f^{*} \overline{N E}(X)=\overline{N E}(Y)\left(\text { resp. } f_{*} \overline{N E}(Y)=\overline{N E}(X)\right) .
$$

(2) Moreover, if $f$ is a finite étale covering and the canonical bundle $K_{X}$ of $X$ is not nef, there is a one-to-one correspondence between the set of extremal rays of $\overline{N E}(X)$ and the set of extremal rays of $\overline{N E}(Y)$.

Proof. (1) is derived from Proposition 4.1.

(2) By Mori's cone theorem ([Mo1], [KM]), we have $\overline{N E}(X)=\sum_{i} R_{i}+$ $\overline{N E}_{K_{X} \geq 0}(X)$, where $R_{i}$ 's are extremal rays of $\overline{N E}(X)$ and $\overline{N E}_{K_{X} \geq 0}(X):=$ $\left\{z \in \overline{N E}(X),\left(z, K_{X}\right) \geq 0\right\}$. We have $\overline{N E}(Y)=\sum_{j} \tilde{R}_{j}+\overline{N E}_{K_{Y} \geq 0}(Y)$ as well. Since $K_{Y} \sim f^{*} K_{X}$ for any $z \in \overline{N E}(X)$, we have $\left(K_{Y}, f^{*} z\right) \geq 0$ (resp. $<0$ ) if and only if $\left(K_{X}, z\right) \geq 0$ (resp. $\left.<0\right)$. Hence by $(1), f^{*}$ and $f_{*}$ induce a bijection between $\overline{N E}_{K_{X} \geq 0}(X)$ and $\overline{N E}_{K_{Y} \geq 0}(Y)$. We have $\overline{N E}(Y)=$ $f^{*} \overline{N E}(X)=\sum_{i} f^{*} R_{i}+\overline{N E}_{K_{Y} \geq 0}(Y)$, where $f^{*} R_{i}$ 's are $K_{Y}$-negative. Since an edge of $\overline{N E}(X)$ is mapped to an edge of $\overline{N E}(Y)$ by $f^{*}, f^{*} R_{i}$ 's are also extremal rays of $\overline{N E}(Y)$. By completely the same method as above, $f_{*} \tilde{R}_{j}$ 's are also extremal rays of $\overline{N E}(X)$. By [Mo1], each extremal ray $R$ of $\overline{N E}(X)$ is spanned by an extremal rational curve $\ell$ on $X$ such that $0<\left(\ell,-K_{X}\right) \leq n+1$. Since $f^{*} R$ is also an extremal ray of $\overline{N E}(Y)$, each irreducible component $\tilde{\ell}_{i}$ of $f^{-1}(\ell)$ spans $f^{*} R$. Therefore we have $f_{*} f^{*} R=R$. Similarly we have $f^{*} f_{*} \tilde{R}=\tilde{R}$ for each extremal ray $\tilde{R}$ of $\overline{N E}(Y)$. Hence the claim follows.

Remark. (1) We shall give another proof of Proposition 3.1 by using (4.2). Since $\kappa(S) \geq 0$, each extremal ray $R$ of $\overline{N E}(S)$ is spanned by a unique $(-1)$-curve $e$. Hence by Proposition $4.2, f^{*}$ and $f_{*}$ give a permutation of the set of $(-1)$-curves on $S$. Since $f$ is a finite étale covering and $e$ is simply connected, $f^{-1}(e)$ consists of $d:=\operatorname{deg}(f)(\geq 2)$ disjoint $(-1)$-curves. This is a contradiction.

(2) If we drop the assumption that $f$ is étale, Proposition $4.2(2)$ does not necessarily hold. For example, let $A$ be an abelian surface and $i \in \operatorname{Aut}(A)$ the group inversion. Let $\hat{A}$ be the surface obtained by blowing-up $A$ at the 16 fixed points of $i$. If we put $X:=\operatorname{Km}(A)$ (i.e. the Kummer surface associated to $A)$ and $Y:=\hat{A}, f: Y \rightarrow X$ is a double covering branched along disjoint (-2)-curves $C_{i}$ 's $(1 \leq i \leq 16)$ on $X$ and $f^{-1}\left(C_{i}\right)$ is a $(-1)$-curve on $Y$. We have $\rho(Y)=\rho(X)=\rho(A)+16$, but $K_{X}$ is trivial and there are no extremal rational curves on $X$. 
Proposition 4.3. Let $f: Y \rightarrow X$ be a finite surjective morphism between smooth projective $n$-folds and assume that $K_{Y}$ is not nef but $K_{X}$ is nef. Then for the contraction morphism $\varphi:=$ Cont $_{\ell}: Y \rightarrow Y^{\prime}$ associated to an extremal ray $\ell$ of $\overline{N E}(Y)$, we have $\operatorname{Supp}(E) \subset \operatorname{Supp}(R)$, where $E$ is the exceptional set of $\varphi$ and $R$ is the ramification divisor of $f: Y \rightarrow X$.

Proof. Since $K_{Y} \sim f^{*} K_{X}+R$, we have $0>\left(K_{Y}, \ell\right)=\left(K_{X}, f_{*} \ell\right)+(R, \ell)$. Since $K_{X}$ is nef by hypotheses, we have $(R, \ell)<0$, hence the claim follows.

Example. In Proposition 4.2 and Remark $2, \widehat{A} \rightarrow X$ is the double covering ramifying along $16(-1)$-curves on $\widehat{A}$.

The following result is a key step towards the construction of a minimal reduction.

Proposition 4.4. Let $f: Y \rightarrow X$ be a nonisomorphic finite étale covering between smooth projective 3 -folds $X$ and $Y$. Suppose that $\kappa(X) \geq 0$ and $\rho(X)=\rho(Y)$. If the canonical bundle $K_{X}$ of $X$ is not nef, all the extremal rays $R$ of $\overline{N E}(X)$ and all the extremal rays $\tilde{R}$ of $\overline{N E}(Y)$ are of type (E1). (cf. [Mo1], $[\mathrm{KM}])$.

(1) The extremal contraction $\operatorname{Cont}_{R}: X \rightarrow X^{\prime}\left(\right.$ resp. Cont $\left._{\tilde{R}}: Y \rightarrow Y^{\prime}\right)$, associated to $R$ (resp. $\tilde{R}$ ), is a divisorial contraction which is (the inverse of) the blow-up along a smooth curve $C$ (resp. $\tilde{C})$ on $X^{\prime}$ (resp. $\left.Y^{\prime}\right)$. Neither $C$ nor $\tilde{C}$ is $\mathbf{P}^{1}$.

(2) Moreover, if $f^{*} R=\tilde{R}, f: Y \rightarrow X$ induces a nonisomorphic finite étale covering $f^{\prime}: Y^{\prime} \rightarrow X^{\prime}$ such that $f^{\prime} \circ \operatorname{Cont}_{\tilde{R}}=\operatorname{Cont}_{R} \circ f$. And set-theoretically, we have $f^{\prime-1}(C)=\tilde{C}$, which is irreducible.

Proof. Since both $X$ and $Y$ are smooth projective 3-folds with nonnegative Kodaira dimension, all the extremal contractions are divisorial contractions. In [Mo1], extremal divisorial contractions of nonsingular projective 3 -folds are classified into 5 types. In our situation, we shall exclude 4 cases (called type $(\mathrm{E} 2) \sim(\mathrm{E} 5)$ in $[\mathrm{KM}])$, where a prime divisor is contracted to a point. By Proposition 4.2 , we may assume that $\tilde{R}=f^{*} R$. Let $E$ (resp. $\tilde{E}$ ) be the exceptional divisor of $\varphi:=\operatorname{Cont}_{R}\left(\operatorname{resp} . \psi:=\operatorname{Cont}_{\tilde{R}}\right)$.

Lemma 4.5. We have $f^{-1}(E)=\tilde{E}$ set-theoretically.

Proof. For each $x \in E$, take an extremal rational curve $C$ which is contained in $\varphi^{-1}(\varphi(x))$ and passes through $x$. $[C]$ spans the extremal ray $R$ of $\overline{N E}(X)$. Since $f$ is a finite étale covering and $C \cong \mathbf{P}^{1}$ is simply connected, 
$f^{-1}(C)$ consists of $C_{i}$ 's $(1 \leq i \leq d)$ which are disjoint unions of $\mathbf{P}^{1}$ 's. Since $\tilde{R}:=f^{*} R$ is also an extremal ray of $\overline{N E}(Y)=f^{*} \overline{N E}(X)$, hence each $\left[C_{i}\right]$ spans $\tilde{R}$ and $C_{i}$ is contracted to a point by $\psi$. Hence $C_{i}$ is contained in $\tilde{E}$. Therefore there exists an inclusion $f^{-1}(x) \subset f^{-1}(C) \subset \tilde{E}$ for all $x \in E$, and so $f^{-1}(E) \subset \tilde{E}$. Since $f$ is a finite étale covering and $\tilde{E}$ is irreducible, we have $f^{-1}(E)=\tilde{E}$ set-theoretically.

Step 1. First we show that each extremal ray $R$ of $\overline{N E}(X)$ is of type (E1). Assume the contrary. Then $\operatorname{Supp}(E)$ is either $\mathbf{P}^{2}$, a smooth quadric or a singular quadric surface, hence is simply connected. Since $f$ is a nonisomorphic finite étale covering, $f^{-1}(E)$ consists of $d(:=\operatorname{deg}(f) \geq 2)$ connected components. However, by Lemma $4.5, f^{-1}(E)=\tilde{E}$ is irreducible. This is a contradiction.

Step 2. Next we show that each extremal ray $\tilde{R}$ of $\overline{N E}(Y)$ is of type (E1). Assume the contrary. For arbitrary 2 points $x$ and $y$ on $E$, we choose 2 points $x^{\prime}, y^{\prime}$ on $\tilde{E}$ so that $f\left(x^{\prime}\right)=x$ and $f\left(y^{\prime}\right)=y$. Since an exceptional divisor $\tilde{E}$ is contracted to a point by $\psi, x^{\prime}$ and $y^{\prime}$ on $E$ can be connected by a chain of extremal rational curves on $Y$ which span $\tilde{R}$. Since $f_{*} \tilde{R}=R, x$ and $y$ on $E$ can also be connected by a chain of extremal rational curves on $X$ which spans $R$. Hence $E$ is contracted to a point by $\varphi$ and this contradicts Step 1 .

Step 3. The former part of (4.4) follows from Steps 1 and 2. For all $p \in \tilde{C}$, the extremal rational curve $\psi^{-1}(p)$ which spans $\tilde{R}$ is mapped to a point by $\varphi \circ f$, since $f_{*} \tilde{R}=R$. Since $Y^{\prime}$ is nonsingular (hence normal), $f: Y \rightarrow X$ induces a surjective morphism $f^{\prime}: Y^{\prime} \rightarrow X^{\prime}$, which is also a nonisomorphic finite étale covering by the purity of branch loci. By Lemma 4.5, we have $f^{\prime-1}(C)=\tilde{C}$ set-theoretically. Finally assume that $C$ is a nonsingular rational curve. Since $C$ is simply connected and $f^{\prime}$ is a finite étale covering with $d:=\operatorname{deg}(f)>1$, $\tilde{C}=f^{\prime-1}(C)$ is not connected. This contradicts the irreducibility of $\tilde{C}$. Thus we have finished the proof of (4.4).

We recall the following general fact for later use.

Proposition 4.6. $\quad$ Let $X$ be a nonsingular projective 3 -fold with $\kappa(X) \geq$ 0 . Then there are only finitely many extremal rays of $\overline{N E}(X)$.

Proof. Since $\kappa(X) \geq 0$, we have $\left|a K_{X}\right| \neq \emptyset$ for some positive integer a. Take an element $D \in\left|a K_{X}\right|$ and let $D=\sum_{i=1}^{N} m_{i} D_{i}$ be the irreducible decomposition. Note that the contraction morphism $\operatorname{Cont}_{R}$, associated to each extremal ray $R$ of $\overline{N E}(X)$, is a divisorial contraction, since $X$ is a smooth 
projective 3 -fold with $\kappa(X) \geq 0$ (cf. [Mo1]). Since $\left(K_{X}, \ell\right)<0$ for any extremal rational curve $\ell$, we have $\left(\ell, D_{i}\right)<0$ and $\ell$ is contained in $D_{i}$ for some $1 \leq i \leq$ $N$. Let $E$ be the exceptional divisor of Cont $\ell$. Since $\ell$ moves and sweeps out $E$, $E$ is contained in $D_{i}$ and hence $E=D_{i}$ by the irreducibility of $D_{i}$. The proof is by contradiction. Assume that there exist an infinite number of extremal rays $R_{i}$ 's $(i=1,2, \ldots)$ on $X$. Let $\ell_{i}$ be the extremal rational curve which spans the extremal ray $R_{i}$ and $E_{i}$ the exceptional divisor of $\operatorname{Cont}_{R_{i}}$ for each $i$. Then there exists some $i(1 \leq i \leq N)$ such that $D_{i}=E_{j}$ for infinitely many $j \in \Lambda$. Assume that some extremal ray $R_{k}(k \in \Lambda)$ is not of type (E1). Then all the irreducible curves in $D_{i}\left(=E_{k}\right)$ are contracted to a point by the contraction morphism of $R_{k}$. Hence they span the same extremal ray $R_{k}$. Since $D_{i}=E_{k}=E_{j}$ for all $j \in \Lambda$, we have $R_{k}=R_{j}$ for infinitely many $j \in \Lambda$. This is a contradiction. Hence all the extremal rays $R_{j}$ 's are of type (E1). Then by the contraction morphism of $R_{j}(j \in \Lambda), D_{i}$ has infinitely many different $\mathbf{P}^{1}$-bundle structures over $\mathbf{P}^{1}$. Again, this is a contradiction.

Proposition 4.7. $\quad$ Let $X$ be a smooth projective 3 -fold with $\kappa(X) \geq 0$. For extremal rays $R$ and $R^{\prime}$ of $\overline{N E}(X)$, suppose:

(1) The extremal contraction $\varphi: X \rightarrow X^{\prime}$ (resp. $\left.\varphi^{\prime}: X \rightarrow X^{\prime \prime}\right)$ associated to $R$ (resp. $R^{\prime}$ ) is (the inverse of) the blow-up along a nonsingular curve $C^{\prime}\left(\right.$ resp. $\left.C^{\prime \prime}\right)$ on $X^{\prime}$ (resp. $\left.X^{\prime \prime}\right)$.

(2) Neither $C^{\prime}$ nor $C^{\prime \prime}$ is $\mathbf{P}^{1}$.

Then we have either $D=E$ or $D \cap E=\emptyset$, where $D$ and $E$ are the exceptional divisors of $\varphi$ and $\varphi^{\prime}$ respectively.

Proof. The proof is by contradiction. Let $\ell$ (resp. $L$ ) be a nonsingular rational curve on $X$ which spans $R$ (resp. $R^{\prime}$ ). Assume that $D \neq E$ and $D \cap E \neq \emptyset$. By assumption, we have $\left(K_{X}, \ell\right)=\left(K_{X}, L\right)=-1,(D, \ell)=$ $(E, L)=-1,(D, L)>0$ and $(E, \ell)>0$. Since $\kappa(X) \geq 0, a K_{X}$ is effective for some positive integer $a$. We claim that $\left(P_{n}\right): a K_{X}-n D-n E$ is effective for all $n \in Z_{\geq 0}$. And clearly this derives a contradiction. The claim is trivial for $n=0$ by assumption. Assume that $\left(P_{n}\right)$ is true. Since $\left(a K_{X}-n D-n E, \ell\right)=$ $-a-n((E, \ell)-1) \leq-a<0$ and $\ell$ moves and sweeps out $D, a K_{X}-(n+1) D-n E$ is effective.

And since $\left(a K_{X}-(n+1) D-n E, L\right)=-a-(n+1)(D, L)+n<-a<0$ and $L$ moves and sweeps out $E, a K_{X}-(n+1) D-(n+1) E$ is also effective. Hence $\left(P_{n+1}\right)$ holds true and we have thus proved the claim.

Our fundamental construction is given in the following theorem. 
Theorem 4.8. Let $f: X \rightarrow X$ be a nontrivial surjective endomorphism of a smooth projective 3 -fold with $\kappa(X) \geq 0$. If $K_{X}$ is not nef, the extremal contraction $\operatorname{Cont}_{R}: X \rightarrow X^{\prime}$ associated to each extremal ray $R$ of $\overline{N E}(X)$ is a divisorial contraction which is (the inverse of) the blow-up along a smooth elliptic curve $E$ on $X^{\prime}$.

Proof. By Proposition 4.2, $f_{*}$ induces a permutation of the set of extremal rays of $\overline{N E}(X)$. By Proposition 4.6, a suitable power $g:=f^{k}(k>0)$ of $f$ has the property that $g_{*} R=R$ for each extremal ray $R$. Hence we may assume from the beginning that $f_{*} R=R$ for each extremal ray $R$ of $\overline{N E}(X)$. By Proposition 4.4, each extremal ray $R$ is of type (E1), that is, the contraction

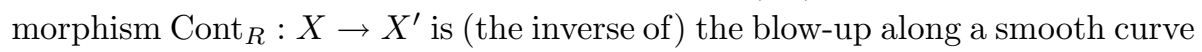
$C$ on $X^{\prime}$ which is not rational. $f$ induces a nontrivial surjective endomorphism $f^{\prime}: X^{\prime} \rightarrow X^{\prime}$ of $X^{\prime}$ such that $f^{\prime-1}(C)=C$ set-theoretically. If $g(C)>1$, $\left.f^{\prime}\right|_{C}: C \rightarrow C$ is an isomorphism. Hence we have $\operatorname{deg}\left(f^{\prime}\right)=\operatorname{deg}\left(\left.f^{\prime}\right|_{C}\right)=1$. This contradicts the nontriviality of $f^{\prime}: X^{\prime} \rightarrow X^{\prime}$. Therefore, $C$ is an elliptic curve.

The following result will be used in Section 7 .

Proposition 4.9. Let $Y_{1} \stackrel{f_{1}}{\longrightarrow} Y_{2} \stackrel{f_{2}}{\longrightarrow} \cdots Y_{n} \stackrel{f_{n}}{\longrightarrow} Y_{n+1} \rightarrow \cdots$ be an infinite sequence of nonisomorphic finite étale coverings between smooth projective 3 -folds $Y_{n}$ 's with non-negative Kodaira dimension $(n=1,2, \cdots)$. Moreover, we assume that the canonical bundle $K_{Y_{n}}$ of $Y_{n}$ is not nef and the Picard numbers $\rho\left(Y_{n}\right)$ of $Y_{n}$ 's are constant. Then each extremal ray $R_{n}$ of $\overline{N E}\left(Y_{n}\right)$ is of type (E1), that is, the extremal contraction $\operatorname{Cont}_{R_{n}}: Y_{n} \rightarrow Z_{n}$ associated to $R_{n}$ is a divisorial contraction which is (the inverse of) the blow-up along a smooth elliptic curve $C_{n}$ on $Z_{n}$.

Proof. This follows easily from Proposition 4.4 and a similar argument to Theorem 4.8 based on the Hurwitz formula for the centers of blow-up.

Next, we shall consider some applications of Proposition 4.2. Theorem 4.10 to Corollary 4.14 will not be used in later sections, but it may be of independent interest. The following theorem may also be considered as a slight generalization of Proposition 4.9 .

Theorem 4.10. Let $X$ be a smooth projective $m$-fold with $\kappa(X) \geq 0$ and $W$ a smooth subvariety of $X$ with $a:=\operatorname{codim}_{X} W \geq 2$. Assume that $W$ enjoys the following property $(E)$ : There exists no infinite descending sequence 


$$
W \stackrel{f_{0}}{\longrightarrow} W_{1} \stackrel{f_{1}}{\longrightarrow} W_{2} \stackrel{f_{2}}{\longrightarrow} \cdots \stackrel{f_{n-1}}{\longrightarrow} W_{n} \stackrel{f_{n}}{\longrightarrow} W_{n+1} \rightarrow \cdots,
$$

where $f_{n}$ are nonisomorphic finite étale coverings. Then $Y:=\mathrm{Bl}_{W}(X)$ (i.e. the blowing-up of $X$ along $W$ ) has no nontrivial surjective endomorphisms.

Corollary 4.11. Let $X$ be a smooth projective $m$-fold with $\kappa(X) \geq 0$ and $W$ a smooth submanifold of $X$ with $\operatorname{codim}_{X} W=: a \geq 2$. If $W$ satisfies the following conditions:

(a) $W$ is of general type, or (b) $\chi\left(\mathscr{O}_{W}\right) \neq 0$, then $Y:=\mathrm{Bl}_{W}(X)$ has no nontrivial surjective endomorphism.

Remark. If $W$ is a point, a Fano manifold or more generally a rationally connected manifold, we have $\chi\left(\mathscr{O}_{W}\right)=1$ by [KoMiMo] and the condition (b) is automatically satisfied. Hence $Y$ has no nontrivial surjective endomorphism.

Proof of Theorem 4.10. Assume that $Y$ has a nontrivial surjective endomorphism $g: Y \rightarrow Y$ and we shall derive a contradiction. Let $\mu: Y \rightarrow X$ be the blowing-up of $X$ along $W$ and $E:=\operatorname{Exc}(\mu)$ the exceptional divisor of $\mu$. For each point $P \in W$, every line in $\mu^{-1}(P) \cong \mathbf{P}^{a-1}$ spans an extremal ray $R$ of $\overline{N E(X)}$ and $\mu=\operatorname{Cont}_{R}$. By Proposition $4.2, g^{*}$ and $g_{*}$ give a permutation of the set of extremal rays of $\overline{N E}(X)$. Let $\mu_{1}$ be the extremal contraction associated to the extremal ray $R^{\prime}:=g_{*} R$.

Step 1. First we show that for each $P \in W, g\left(\mu^{-1}(P)\right)$ is contracted to a point by $\mu_{1}$.

Proof. The restriction $g: \mu^{-1}(P) \cong \mathbf{P}^{a-1} \rightarrow g\left(\mu^{-1}(P)\right)$ is also a finite étale covering. Hence $g\left(\mu^{-1}(P)\right)$ is also nonsingular and since $1=\chi\left(\mathscr{O}_{\mu^{-1}(P)}\right)=$ $\operatorname{deg}\left(g \mid \mu^{-1}(P)\right) \times \chi\left(\mathscr{O}_{g^{-1}(P)}\right), g \mid \mu^{-1}(P)$ is an isomorphism and $g\left(\mu^{-1}(P)\right) \cong$ $\mathbf{P}^{a-1}$. It suffices to show that any two points $x, y \in g\left(\mu^{-1}(P)\right)$ can be connected by an irreducible extremal rational curve which spans the extremal ray $R^{\prime}$. Let $\ell^{\prime}$ be the line in $g\left(\mu^{-1}(P)\right) \cong \mathbf{P}^{a-1}$ passing through $x$ and $y$. Then $\ell$, which is the inverse image of $\ell^{\prime}$ by $g \mid \mu^{-1}(P)$, is a line in $\mu^{-1}(P) \cong \mathbf{P}^{a-1}$, hence spans the extremal ray $R$. Therefore $\ell^{\prime}=g(\ell)$ also spans the extremal ray $R^{\prime}:=g_{*} R$.

Step 2. $\mu_{1}:=\operatorname{Cont}_{R^{\prime}}$ is a divisorial contraction with $\operatorname{Supp}\left(\operatorname{Exc}\left(\mu_{1}\right)\right)=$ $g(E)$ and we have the following commutative diagram:

$$
\begin{aligned}
& g(E)=: E_{1} \subset Y \stackrel{g}{\longleftarrow} Y \supset E \\
& \downarrow \mu_{1} \downarrow \quad \downarrow \mu \downarrow \\
& h(W)=: W_{1} \subset X_{1} \stackrel{h}{\longleftarrow} X \supset W,
\end{aligned}
$$


where $h: X \rightarrow X_{1}$ is a nonisomorphic finite étale covering and $h^{-1}\left(W_{1}\right)=W$ set-theoretically.

Proof. By Step 1, $\mu_{1} \circ g \circ \mu^{-1}(x)$ is a point for all $x \in X$. Hence there exists a morphism $h: X \rightarrow X_{1}$ which satisfies the above diagram. Since $\mu_{1}(g(E))=h(W)$ and $\operatorname{dim}(g(E))=\operatorname{dim}(E)>\operatorname{dim}(W) \geq \operatorname{dim}(h(W))$, the divisor $g(E)$ is contained in $\operatorname{Supp}\left(\operatorname{Exc}\left(\mu_{1}\right)\right)$ and $\mu_{1}$ is a divisorial contraction. Since $\operatorname{Exc}\left(\mu_{1}\right)$ is a prime divisor, we have $\operatorname{Supp}\left(\operatorname{Exc}\left(\mu_{1}\right)\right)=g(E)$. By the same argument as in the proof of Lemma 4.5 , we have $\operatorname{Supp}\left(g^{-1}\left(E_{1}\right)\right)=E$.

Next, we show that $h: X \rightarrow X_{1}$ is a finite morphism. Assume the contrary. Then for some $x \in X_{1}$, we have $\operatorname{dim}\left(h^{-1}(x)\right)>0$. Since $g$ is finite, we have $x \in W_{1}$. Since $g^{-1}\left(E_{1}\right)=E$, we have $h^{-1}(x) \subset W$. Take an irreducible component $\Delta$ of $h^{-1}(x)$ such that $\operatorname{dim}(\Delta) \geq 1$. Let $P, Q \in \mu^{-1}(\Delta)$ be 2 points not contained in the same fiber of $\mu \mid \mu^{-1}(\Delta): \mu^{-1}(\Delta) \rightarrow \Delta$ and $C$ an irreducible curve in $\mu^{-1}(\Delta)$ passing through $P$ and $Q$. Since $C^{\prime}:=g(C)$ is an irreducible curve in $g\left(\mu^{-1}(\Delta)\right)$ and contracted to a point $x$ by $\mu_{1}, C^{\prime}$ spans the extremal ray $R^{\prime}$. Hence $C$ also spans the extremal ray $R=g^{*} R^{\prime}$ and is contracted to a point by $\mu$. This is a contradiction. The latter will be proved in Step 3.

Step 3. $\mu_{1}:=\operatorname{Cont}_{R^{\prime}}: Y \rightarrow X_{1}$ is the Nakano-Fujiki's contraction. (That is, $Y$ is the blowing-up of a smooth $m$-fold $X_{1}$ along a submanifold $W_{1}:=h(W)$ of codimension $a$.)

Proof. Since $E$ is nonsingular and $g$ is a finite étale morphism, $E_{1}:=g(E)$ is nonsingular. Each fiber of $\mu_{1} \mid g(E): g(E) \rightarrow h(W)$ is isomorphic to $\mathbf{P}^{a-1}$ by Steps 1 and 2. Hence $g(E)$ is a $\mathbf{P}^{a-1}$-bundle on $h(W)$ and $h(W)$ is also nonsingular. Since $g$ is finite étale and $g^{-1}\left(E_{1}\right)=E$ set-theoretically, we have $g^{*} E_{1}=E$. Hence for all $P \in h(W)$, we have $\left[E_{1}\right] \mid \mu_{1}^{-1}(p) \cong \mathscr{O}_{P^{a-1}}(-1)$ by the projection formula. Therefore $\mu_{1}$ coincides with the Nakano-Fujiki's contraction and $X_{1}$ is nonsingular. The finite morphism $h: X \rightarrow X_{1}$ between smooth projective $m$-folds $X$ and $X_{1}$ is unramified outside $W$ with $\operatorname{codim}_{X} W \geq 2$. Hence $h_{1}$ is unramified by the purity of branch loci. Since $g^{*} E_{1}=E$, we have $h^{-1}\left(W_{1}\right)=W$ set-theoretically and in particular, they are connected.

Step 4. By applying the same argument as in Steps 1, 2 and 3 repeatedly, we obtain the following commutative diagram: 


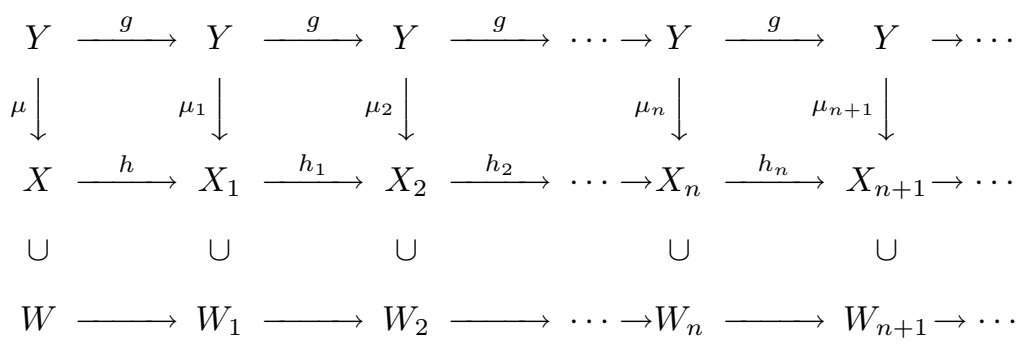

where

(1) $h_{n}: X_{n} \rightarrow X_{n+1}(n=0,1,2, \cdots)$ is an infinite sequence of nonisomorphic finite étale coverings between smooth projective $m$-folds $X_{n}$ 's with $\kappa\left(X_{n}\right) \geq 0$ (here we put $X_{0}:=X$ ),

(2) $\mu_{n}: Y \rightarrow X_{n}$ is the blowing-up along a smooth, connected submanifold $W_{n}$ of $X_{n}$ with $\operatorname{codim}_{X_{n}} W_{n}=a$,

(3) $h^{-1}\left(W_{n+1}\right)=W_{n}$ set-theoretically for all $n$.

By assumption, there exists $p \in N$ such that $\left.h_{n}\right|_{W_{n}}: W_{n} \cong W_{n+1}$ is an isomorphism for all $n \geq p$. Hence by (3), we have $\operatorname{deg}\left(h_{n}\right)=\operatorname{deg}\left(h_{n} \mid W_{n}\right)=1$ for all $n \geq p$ and $h_{n}: X_{n} \rightarrow X_{n+1}$ is an isomorphism. This is a contradiction.

Proof of Corollary 4.11. It is enough to show that $W$ satisfies the property (E) under the above assumption. First we consider the case (a). Recall the following theorem.

Theorem [Kol 2, Proposition 9.4]. Let $h: Y \rightarrow X$ be a finite étale covering between smooth projective varieties of general type. Then we have $P_{m}(Y)=\operatorname{deg}(h) \times P_{m}(X)$ for all $m \geq 2$.

Assume the contrary. Then there exists an infinite sequence: $W \stackrel{f_{0}}{\longrightarrow}$ $W_{1} \stackrel{f_{1}}{\longrightarrow} W_{2} \stackrel{f_{2}}{\longrightarrow} \cdots \rightarrow W_{n} \stackrel{f_{n}}{\longrightarrow} \cdots$, where $f_{n}$ are nonisomorphic finite étale covering between smooth projective $(m-a)$-folds $W_{n}$ 's of general type. Fix a large positive integer $k$ such that $P_{k}(W)>0$. Then by Kollár's theorem, we have $P_{k}(W)=\prod_{i=0}^{n} \operatorname{deg}\left(f_{i}\right) \times P_{k}\left(W_{n+1}\right)$. Since $\operatorname{deg}\left(f_{i}\right)>1$ for all $i$, we have $P_{k}(W)=\infty$ if we let $n \rightarrow \infty$. This is a contradiction. The proof in the case (b) is almost the same as above, so we omit it.

Remark. Theorem 4.10 does not necessarily hold if $W$ does not satisfy the property $(\mathrm{E})$. We shall give such an example. 
Let $S$ be a smooth algebraic surface with $\kappa(S) \geq 0$ which admits a nontrivial surjective endomorphism $g$ as in Theorem 3.2. Let $Y$ be an arbitrary smooth projective $n$-fold with $\kappa(Y) \geq 0$ and consider the direct product $Z:=Y \times S$. Fix a point $P$ on $Y$ and let $X$ be a smooth projective $(n+2)$-fold which is obtained by blowing-up $Z$ along $W:=\{P\} \times S(\cong S)$. We have $X \cong Y^{\prime} \times S$, where $Y^{\prime}$ is the blowing-up of $Y$ at a point $P \in Y$. Then $f:=\operatorname{id}_{Y^{\prime}} \times g: X \rightarrow X$ is a nontrivial surjective endomorphism of $X$.

Most of Proposition 4.4 holds in a more general case.

Proposition 4.12. Under the same assumption as in Proposition 4.2 (2), for each extremal ray $R$ of $\overline{N E}(X)$, we have the following commutative diagram:

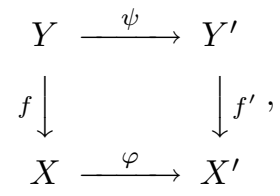

where $\varphi:=\operatorname{Cont}_{R}: X \rightarrow X^{\prime}\left(\right.$ resp. $\left.\psi:=\operatorname{Cont}_{\tilde{R}}: Y \rightarrow Y^{\prime}\right)$ is the extremal contraction associated to $R$ (resp. $\tilde{R}:=f^{*} R$ ) and $f^{\prime}: Y^{\prime} \rightarrow X^{\prime}$ is a finite surjective morphism. Moreover,

(1) $\varphi$ is birational if and only if $\psi$ is birational.

(2) $\varphi$ is a divisorial contraction if and only if $\psi$ is a divisorial contraction.

The proof is almost the same as in Proposition 4.4, so we omit it. We shall give some other applications of Proposition 4.2.

Theorem 4.13. Let $S$ and $T$ be relatively minimal rational elliptic surfaces and $\varphi: S \rightarrow T$ a surjective morphism. Then $\varphi$ is an isomorphism.

Proof. Since $\rho(S)=\rho(T)=10, \varphi: S \rightarrow T$ is a finite morphism by Lemma 2.3. $S$ and $T$ are obtained as 9-points blowing-up of $\mathbf{P}^{2}$ and they are simply connected. Hence if $\varphi$ is unramified, $\varphi$ is an isomorphism. Hence we may assume that $\varphi$ is ramified and shall derive a contradiction. $S$ (resp. $T$ ) has the unique structure of an elliptic surface $p: S \rightarrow \mathbf{P}^{1}$ (resp. $q: T \rightarrow \mathbf{P}^{1}$ ) with at most one multiple fiber $m D$ (resp. $n E$ ) of type ${ }_{m} I_{b}$ (resp. ${ }_{n} I_{b^{\prime}}$ ), where $m \geq 1, b \geq 0$. (resp. $n \geq 1, b^{\prime} \geq 0$ ) (See [Fu1], [HL].) In the case where $m=1$ (resp. $n=1), S$ (resp. $T$ ) is a rational elliptic surface with a global section.

Step 1. First we show that $\varphi: S \rightarrow T$ preserves the structure of an elliptic fibration of $S$ and $T$, and the ramification divisor $\Delta$ of $\varphi$ is supported on fibers 
of $p: S \rightarrow \mathbf{P}^{1}$. By the canonical bundle formula of Kodaira, we have

(*) $\quad K_{S} \sim-D, K_{T} \sim-E$, where $m D \in\left|p^{*} \mathscr{O}_{P^{1}}(1)\right|$ and $n E \in\left|q^{*} \mathscr{O}_{P^{1}}(1)\right|$.

In particular, $D$ (resp. $E$ ) is nef. We have $(\star) K_{S} \sim \varphi^{*} K_{T}+\Delta$, where $\Delta$ is the ramification divisor of $\varphi$. (*) and $(\star)$ implies that $(\xi) D \sim \varphi^{*} E-\Delta$. Since $0=$ $D^{2}=\Delta^{2}-2\left(E, \varphi_{*} \Delta\right)$ and $E$ is nef, we have $\Delta^{2} \geq 0$. On the other hand, we have $\Delta^{2}=\left(\varphi^{*} E-D\right)^{2}=-2\left(E, \varphi_{*} D\right) \leq 0$. Hence $\Delta^{2}=0,\left(\varphi^{*} E, \Delta\right)=\left(E, \varphi_{*} D\right)=0$ and $\operatorname{Supp}\left(\varphi_{*} D\right)$ is contained in fibers of $q$. Therefore $\varphi: S \rightarrow T$ maps each fiber of $p$ to some fiber of $q$ and preserves the elliptic fiber space structures of $S$ and $T$. ( $(\tau)$ implies that $(D, \Delta)=0$, hence $\operatorname{Supp}(\Delta)$ is contained in fibers of $p$. Since $\Delta^{2}=0$, we have $\Delta \sim_{Q} \alpha f$, where $f$ is a general fiber of $p: S \rightarrow \mathbf{P}^{1}$ and $\alpha$ is a positive rational number. By $(\mathcal{\zeta})$, we have $\varphi^{*} E \sim_{Q}(\alpha+1 / m) f$. Hence we have $(※) \varphi^{*} F \sim_{Q} n(\alpha+1 / m) f$, where $F$ is a general fiber of $q: T \rightarrow \mathbf{P}^{1}$.

Step 2. Next, we show that $\varphi^{*}$ and $\varphi_{*}$ give a one-to-one correspondence between the set of extremal rays on $\overline{N E}(S)$ and the set of extremal rays on $\overline{N E}(T)$. Since $\rho(S)=\rho(T)=10, \varphi_{*}$ and $\varphi^{*}$ give a bijection between $\overline{N E}(S)$ and $\overline{N E}(T)$. By the cone theorem [Mo1], [KM], we have $\overline{N E}(S)=\sum_{i} \tilde{R}_{i}+$ $\overline{N E}_{K_{S} \geq 0}(S)$ and $\overline{N E}(T)=\sum_{j} R_{j}+\overline{N E}_{K_{T} \geq 0}(T)$. Note that each extremal ray $\tilde{R}_{i}$ (resp. $R_{j}$ ) is spanned by a $(-1)$-curve on $S$ (resp. $T$ ). Since $m K_{S} \sim_{Q}-f$ and $n K_{T} \sim_{Q}-F,(※)$ in Step 1 implies that $\varphi^{*}$ and $\varphi_{*}$ induce a bijection between $\overline{N E}_{K_{S} \geq 0}(S)$ (resp. $\left.\overline{N E}_{K_{S}<0}(S)\right)$ and $\overline{N E}_{K_{T} \geq 0}(T)$ (resp. $\overline{N E}_{K_{T}<0}(T)$ ). Then by the same argument as in the proof of Proposition $4.2, \varphi^{*}$ and $\varphi_{*}$ give a one-to-one correspondence between the set of extremal rays of $\overline{N E}(S)$ and the set of extremal rays of $\overline{N E}(T)$.

Step 3. We claim that for each $(-1)$-curve $e$ on $T$, there exists a unique $(-1)$-curve $e^{\prime}$ on $S$ such that $\varphi^{*} e \sim e^{\prime}$. Let $R$ be the extremal ray of $\overline{N E}(T)$ spanned by $[e]$ and $\varphi^{*} e \sim \sum_{j} a_{j} C_{j}$ an irreducible decomposition of $\varphi^{*} e$. By Step $2, R^{\prime}:=\varphi^{*} R$ is also an extremal ray of $\overline{N E}(T)$ and spanned by $\left[\varphi^{*} e\right]$. Hence each $\left[C_{j}\right]$ spans the same extremal ray $R^{\prime}$. We have $\left(C_{j}\right)^{2}<0$ for all $j$. So if $\varphi^{*} e$ is reducible, we have $\left(C_{i}, C_{j}\right)<0$ for $i \neq j$ and this is a contradiction. Hence we have $\varphi^{*} e \sim a e^{\prime}$, where $e^{\prime}$ is a $(-1)$-curve on $S$. If $a>1, e^{\prime}$ is contained in the ramification divisor of $\varphi$, hence is supported in a fiber of $p: S \rightarrow \mathbf{P}^{1}$ by Step 1. However, since the elliptic fibration $p: S \rightarrow \mathbf{P}^{1}$ is relatively minimal, this is a contradiction. Hence $a=1$ and the claim follows.

Step 4. Since $e^{\prime 2}=\operatorname{deg}(\varphi) e^{2}$ and ${e^{\prime 2}}^{2}=e^{2}=-1$, we have $\operatorname{deg}(\varphi)=1$ and $\varphi$ is an isomorphism. This contradicts our assumption that $\varphi$ is ramified. Thus we have completed the proof of Theorem 4.13. 
Corollary 4.14. $\quad$ Let $S$ be a relatively minimal rational elliptic surface. Then $S$ has no nontrivial surjective endomorphism. In particular, $S$ is not a toric surface.

Proof. By [N6, Lemma 4], a toric variety has a nontrivial surjective endomorphism. Hence the claim immediately follows.

Remark. Theorem 4.13 does not hold if we drop the assumption that $\varphi: S \rightarrow T$ is a morphism. We shall give such an example.

Let $S$ be an elliptic modular surface of level 3 structure in the sense of Shioda, that is the universal family of elliptic curves with level 3 structure. It is isomorphic to the Hesse pencil: $x^{3}+y^{3}+z^{3}-3 \lambda x y z=0, \lambda \in \mathbf{P}^{1}$, $(x: y: z) \in \mathbf{P}^{2}$. It has just 4 singular fibers of type $\left(I_{3}\right)$ and 9 global sections. Take an arbitrary section $o . S$ can be considered as an elliptic curve $\mathcal{E}$ over the function field $K:=\boldsymbol{C}\left(\mathbf{P}^{1}\right)$ given with a $K$-rational point $o$. Then the group of $K$-rational points of $\mathcal{E}$ with the origin $o$ can be identified with the set of holomorphic sections of $f: S \rightarrow \mathbf{P}^{1}$. This is called the Mordell-Weil group of $S$ and in this case, isomorphic to $\mathbf{Z} / \mathbf{3 Z} \oplus \mathbf{Z} / \mathbf{3 Z}$. Take a torsion section $s$ of order 3 such that $s$ and $o$ pass through the same irreducible component of the singular fiber $F_{0}$ over $\lambda=1$. Let $G$ be a finite birational automorphism group of $\mathcal{E}$ induced from the translation by $s$ in the group law over $K$. Since $S$ is relatively minimal, $G$ is a subgroup of $\operatorname{Aut}(S)$. The only fixed points of $G$ are the 3 nodes in the singular fiber $F_{0}$ of type $I_{3}$. The quotient space $S / G$ has three $A_{2}$-singularities and the minimal resolution $T$ of $S / G$ is also a rational elliptic surface with a global section. $T$ has three $I_{1}$-type singular fibers and one $I_{9}$-type singular fiber, and the Mordell-Weil group of $T$ is isomorphic to Z/3Z. The quotient map $q: S \rightarrow S / G$ induces a generically finite rational map $q^{\prime}: S \cdots \rightarrow T$ of degree 3 between rational elliptic surfaces $S$ and $T$.

Now we are in a position to construct a minimal reduction. We shall apply the minimal model program (MMP) to the morphism $f: X \rightarrow X$ which is a nontrivial surjective endomorphism of a smooth projective 3 -fold with $\kappa(X) \geq$ 0 . Assume that the canonical bundle $K_{X}$ of $X$ is not nef. Then if we replace $f$ by a suitable power $f^{k}(k>0)$ of $f$, from the beginning, we may assume that $f_{*} R=R$ for each extremal ray $R$ of $\overline{N E}(X)$ by completely the same method as in the proof of Theorem 4.8. By Proposition 4.4 and Theorem 4.8, each extremal ray $R$ is of type (E1) and the contraction morphism $\pi:=\operatorname{Cont}_{R}$ : $X \rightarrow X_{1}$, induces a nontrivial surjective endomorphism $f_{1}: X_{1} \rightarrow X_{1}$ of a smooth projective 3 -fold $X_{1}$ with $\kappa\left(X_{1}\right) \geq 0$ and $\rho\left(X_{1}\right)=\rho(X)-1$ such that $f_{1} \circ \pi=\pi \circ f$. If $K_{X_{1}}$ is not nef, by the same method as above, we can replace 
$f_{1}$ (and hence $f$ ) by a suitable power of $f_{1}$ (hence $f$ ) such that $\left(f_{1}\right)_{*} R_{1}=R_{1}$ (resp. $f_{*} R=R$ ) for each extremal ray $R_{1}$ (resp. $R$ ) of $\overline{N E}\left(X_{1}\right)(\operatorname{resp} . \overline{N E}(X)$ ). We repeat the same procedure as above. Since $\rho(X)>\rho\left(X_{1}\right)>\cdots$, this process terminates after a finite number of steps and eventually, we obtain a nonsingular minimal model $X_{n}$ of $X$ and a nontrivial surjective endomorphism $f_{n}$ of $X_{n}$. To sum up, we have the commutative diagram:

(*)

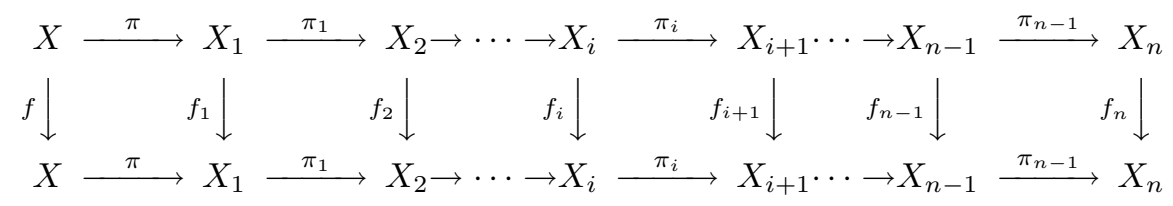

where

(1) $f_{i}$ is a nontrivial surjective endomorphism of a smooth projective 3 -fold $X_{i}$ with $\kappa(X)_{i} \geq 0$,

(2) $\pi_{i-1}: X_{i-1} \rightarrow X_{i}$ is (the inverse of) the blow-up of a smooth elliptic curve $C_{i}$ on $X_{i}$ such that $f_{i}^{-1}\left(C_{i}\right)=C_{i}$ set-theoretically, (here we put $X_{0}=X$ and $\left.\pi_{0}=\pi\right)$

(3) $X_{n}$ is a nonsingular minimal model of $X$.

Definition 4.15. We call $f_{n}: X_{n} \rightarrow X_{n}$ a minimal reduction of $f$ : $X \rightarrow X$.

Corollary 4.16. Let $f: X \rightarrow X$ be a nontrivial surjective endomorphism of a smooth projective 3 -fold with $\kappa(X) \geq 0$. Then all the minimal models of $X$ are nonsingular.

Proof. The minimal reduction $f_{n}: X_{n} \rightarrow X_{n}$ of $f: X \rightarrow X$ is nonsingular by construction. Minimal models of $X$ are isomorphic in codimension 1 and connected by a sequence of flops by [Kaw1] and [Kol1] and 3-dimensional terminal flops preserve the analytic singularity type by [Kol1]. Hence the claim follows.

By the abundance theorem by Miyaoka [Mi1], [Mi2] and Kawamata [Kaw2], the canonical bundle $K_{Y}$ of a minimal reduction $Y:=X_{n}$ is semi-ample and the minimal reduction $f_{n}: Y \rightarrow Y$ of $f: X \rightarrow X$ is compatible with the Iitaka fibration $\phi: Y \rightarrow \phi(Y)$ of $Y$. If $\kappa(X)=0,2$, or $\kappa(X)=1$ and the general fiber of the Iitaka fibration of $Y:=X_{\min }$ is a hyperelliptic surface, then we can describe their structures completely thanks to the Bogomolov's decomposition 
theorem [Be], the standard fibration theorem by Nakayama [N1], [N3] and Fujiki's generic quotient theorem [F2], [F3].

\section{$\S 5 . \quad$ Classifications of Minimal Reductions}

Before stating our main theorem, we need the following definition.

Definition 5.0. Let $f: V \rightarrow S$ be an elliptic fibration from a smooth projective $n$-fold $V$ onto a normal projective variety $S$. It is called a Seifert elliptic fiber space if the following conditions are satisfied:

(1) $f$ is equidimensional.

(2) $K_{V}$ is numerically $f$-trivial.

(3) $V$ is a principal fiber bundle outside the discriminant locus $D$ of $S$ and the singular locus of $S$. Furthermore if $\operatorname{codim}(D)=1$, it has multiple singular fibers of type ${ }_{m} I_{0}(m>1)$ generically along $D$. (i.e. For a generic analytic arc $C$ in $S$ which intersects transversally with $D$ at a point $x, f^{-1}(C) \rightarrow C$ is a nonsingular minimal elliptic surface over $C$ and $f^{-1}(x)$ is a multiple singular fiber of type ${ }_{m} I_{0}$ in the sense of Kodaira, whose support is a nonsingular elliptic curve.)

Remark. From Nakayama's theorem [N3], [N4], it follows that each fiber of $f$ is a nonsingular elliptic curve.

Now, we state MAIN THEOREM.

MAIN THEOREM (A). Let $f: X \rightarrow X$ be a nontrivial surjective endomorphism of a smooth projective 3 -fold $X$ with non-negative Kodaira dimension. Then the minimal models of $X$ are nonsingular and one of the following cases occurs.

Case 1. If $\kappa(X)=2, X$ has the structure of a Seifert elliptic fiber space $\varphi$ : $X \rightarrow T$ over a normal surface $T$ with at most quotient singularities. Moreover,

(1a) After replacing $f$ by a suitable power $f^{k}(k>0)$ of $f, f$ induces an automorphism of the base space $T$ and is compatible with $\varphi$.

(1b) A suitable finite étale covering $\tilde{X}$ of $X$ is isomorphic to the direct product $\tilde{T} \times E$ of an elliptic curve $E$ and a nonsingular (not necessarily minimal) algebraic surface $\tilde{T}$ of general type.

Case 2. If $\kappa(X)=1$, the minimal model $X^{\prime}$ of $X$ is nonsingular and the general fiber of the Iitaka fibration $\varphi: X^{\prime} \rightarrow C$ is isomorphic to an abelian 
surface (Case 2.a) or a hyperelliptic surface (Case 2.b). In the latter (Case 2.b), we obtain the following diagram of fiber spaces: $X \stackrel{g}{\longrightarrow} T \stackrel{h}{\longrightarrow} C$, where $g: X \rightarrow T$ is a Seifert elliptic fiber space over a normal surface $T$ with at most quotient singularities, $h: T \rightarrow C$ is a $\mathbf{P}^{1}$ or elliptic fiber space, and the general fiber of $\phi:=h \circ g$ is a hyperelliptic surface. After replacing $f$ by a suitable power $f^{k}(k>0)$ of $f, f$ induces an endomorphism (resp. an automorphism) of $T$ (resp. $C$ ) and is compatible with $\phi, g$ and $h$. A suitable finite étale covering $\tilde{X}$ of $X$ is isomorphic to the direct product $\tilde{T} \times E$ of an elliptic curve $E$ and a smooth algebraic surface $\tilde{T}$ with $\kappa(\tilde{T})=1$.

Case 3. If $\kappa(X)=0$, a suitable finite étale covering $\tilde{X}$ of $X$ is isomorphic to an abelian 3-fold (Case 3.a) or the direct product $\tilde{T} \times E$ of an elliptic curve $E$ and a nonsingular algebraic surface $\tilde{T}$ which is birational to an abelian surface or a K3 surface (Case 3.b). In the latter (Case 3.b), $X$ has the structure of a Seifert elliptic fiber space over the quotient of $\tilde{T}$ by a finite group $G$. After replacing $f$ by a suitable power $f^{k}(k>0)$ of $f, f$ is compatible with the elliptic fiber space structure and induces an automorphism of the base space $\tilde{T} / G$. Moreover, $X$ has another Seifert fiber space structure over the quotient of $E$ by a finite group $G^{\prime}$ and its general fiber is birational to a K3 surface or an Enriques surface.

In Cases 1, 3 and 2b, the minimal model of $X$ is unique up to isomorphisms.

In this section, we shall study the structure of a minimal smooth projective 3-fold with $\kappa(X) \geq 0$ which admits a nontrivial surjective endomorphism $\varphi$ : $X \rightarrow X$. We have to deal with the problem under the weak assumption that $f: Y \rightarrow X$ is a nonisomorphic finite étale covering between smooth projective 3-folds $X$ and $Y$ which are birationally equivalent and minimal. ( $X$ and $Y$ are isomorphic in codimension 1 and connected by a finite succession of flops by Kawamata [Kaw2] and Kollár [Kol1].)

We first treat the case where $\kappa(X)=\kappa(Y)=2$.

Theorem 5.1. Let $f: Y \rightarrow X$ be a nonisomorphic finite étale covering between minimal smooth projective 3 -folds $X$ and $Y$ which are birationally equivalent and $\kappa(X)=\kappa(Y)=2$. Then

(1) There exists an isomorphism between $X$ and $Y$, and $f$ can be regarded as a nontrivial surjective endomorphism of $X$.

(2) $X$ has the structure of a Seifert elliptic fiber space $\varphi: X \rightarrow T$ over a normal surface $T$ with at most quotient singularities. $f$ induces an automorphism of $T$ such that $\varphi \circ f=f \circ \varphi$. 
(3) A suitable finite étale covering $\tilde{X}$ of $X$ is isomorphic to the direct product $\tilde{T} \times E$ of an elliptic curve $E$ and a nonsingular minimal algebraic surface $\tilde{T}$ of general type.

Lemma 5.2. Let $u: X \cdots \rightarrow X^{\prime}$ be a birational map between smooth projective $n$-folds $X$ and $X^{\prime}$ which is an isomorphism in codimension one. Let $f: Y \rightarrow X$ be a nonisomorphic finite étale covering between smooth projective $n$-folds $X$ and $Y$. Then there exist a smooth projective $n$-fold $Y^{\prime}$ and a nonisomorphic finite étale covering $g: Y^{\prime} \rightarrow X^{\prime}$ which induces the following commutative diagram:

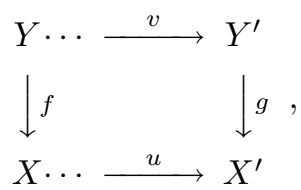

where $v: Y \cdots \rightarrow Y^{\prime}$ is a birational map and an isomorphism in codimension one.

Proof. By assumption, there exist an algebraic subvariety $A$ (resp. $A^{\prime}$ ) of $X$ (resp. $X^{\prime}$ ) with $\operatorname{codim}_{X} A \geq 2$ (resp. $\operatorname{codim}_{X^{\prime}} A^{\prime} \geq 2$ ) and an isomorphism $u: X \backslash A \cong X^{\prime} \backslash A^{\prime}$. If we pull back the finite étale covering $f^{-1}(X \backslash A) \rightarrow X \backslash A$ by $u^{-1}$, we obtain a finite étale covering $f^{\prime}: \overline{X^{\prime} \backslash A^{\prime}} \rightarrow X^{\prime} \backslash A^{\prime}$ over $X^{\prime} \backslash A^{\prime}$. Since $\operatorname{codim}_{X^{\prime}} A^{\prime} \geq 2$, the fundamental group of $X^{\prime} \backslash A^{\prime}$ is isomorphic to the fundamental group of $X^{\prime}$. Hence the covering $f^{\prime}: \overline{X^{\prime} \backslash A^{\prime}} \rightarrow X^{\prime} \backslash A^{\prime}$ can be extended to the finite étale covering $g: Y^{\prime} \rightarrow X^{\prime}$. On the other hand, there is a natural inclusion $\iota: f^{-1}(X \backslash A) \rightarrow Y^{\prime}$ and since $\operatorname{codim}_{X} f^{-1}(X \backslash A) \geq 2$, this can be extended to a birational map $v: Y \cdots \rightarrow Y^{\prime}$ which is an isomorphism in codimension one.

Corollary 5.3. Under the same assumption as in Lemma 5.2, we further assume that $X$ and $Y$ are minimal nonsingular projective 3 -folds and are birationally equivalent. Then $Y^{\prime}$ is also minimal, and $Y^{\prime}$ and $X$ are connected by a finite sequence of flops.

Proof. Since $K_{Y^{\prime}} \sim g^{*} K_{X^{\prime}}$ and $K_{X^{\prime}}$ is nef, $K_{Y^{\prime}}$ is also nef. Both $X$ and $Y^{\prime}$ are birationally equivalent and minimal, hence the claim follows by [Kaw1] and $[\mathrm{Ko} 1]$.

The following theorem due to Nakayama [N3], [N4, Theorems 4.2 and 5.1] plays a key role in this section. 
Theorem (N). Let $f: V \rightarrow S$ be an elliptic fibration from a smooth projective $n$-fold $V$ to a normal projective variety $S$. Suppose that

(1) No prime divisor $\Delta$ of $V$ with $\operatorname{codim} f(\Delta) \geq 2$ is uniruled,

(2) No prime divisor $\Delta$ of $V$ with $\operatorname{codim} f(\Delta)=1$ is covered by a family of rational curves contained in fibers of $f$,

(3) $K_{V}$ is numerically $f$-trivial.

Then there exists a generically finite surjective morphism $T \rightarrow S$ such that

(1) $T$ is a smooth projective variety.

(2) For the normalization $W$ of the main component of $V \times T$, the induced morphism $W \rightarrow V$ is a finite étale covering.

(3) $W$ is isomorphic to the direct product $T \times E$ over $T$ for an elliptic curve $E$.

Remark. In [N3], [N4], Nakayama posed the stronger assumptions that no divisor of $V$ is uniruled if it is not dominating $S$. But his proof works under the weak condition (2).

Proof of Theorem 5.1. Since $\kappa(X)=2$ and $K_{X}$ is nef, by the 3-dimensional abundance theorem due to [Mi1], [Mi2] and [Kaw2], $K_{X}$ is semi-ample. The pluricanonical mapping $\varphi$ associated to the complete linear system $\left|m K_{X}\right|$ gives the Iitaka fibration $\varphi: X \rightarrow S$ for a sufficiently large positive integer $m . S$ is a normal surface with at most quotient singularities and we have $m K_{X} \sim \varphi^{*} L$ for a very ample line bundle $L$ on $S$. In particular, $K_{X}$ is numerically $\varphi$-trivial. By the standard fibration theorem [N1. Appendix A. Theorem A.1], if we perform a finite succession of flops to $X$ and take a suitable birational model $\mu: T \rightarrow S$, we have an equidimensional elliptic fibration $\psi: X_{1} \rightarrow T$ over $T$. Since 3-dimensional terminal flops preserve the analytic singularity type by [Ko1], $X_{1}$ is nonsingular. Since $m K_{X_{1}} \sim(\mu \circ \psi)^{*} L, K_{X_{1}}$ is numerically $\psi$-trivial. By Lemma 5.2, we can take a nonisomorphic finite étale covering $g_{2}: X_{2} \rightarrow X_{1}$ which is birational to $f: Y \rightarrow X$. By Corollary $5.3, X_{2}$ is also minimal and $X_{2}$ and $X$ are connected by a finite sequence of flops. Thus if we apply Lemma 5.2 and Corollary 5.3 successively, the original $f: Y \rightarrow X$ induces an infinite tower of nonisomorphic finite étale coverings $X \underset{\text { bir }}{\sim} X_{1} \stackrel{g_{2}}{\longleftarrow}$ $X_{2} \stackrel{g_{3}}{\longleftarrow} X_{3} \leftarrow \cdots \stackrel{g_{n}}{\longleftarrow} X_{n} \stackrel{g_{n+1}}{\longleftarrow} X_{n+1} \leftarrow \cdots$ between minimal nonsingular projective 3 -folds $X_{n}$ 's, where $\kappa\left(X_{n}\right)=2$ and $X_{n}$ is a minimal model of $X$ for all $n$. Since they are all connected to $X$ by a finite sequence of flops $v_{n}: X \cdots \rightarrow X_{n}$, we have a natural isomorphism $v_{n}^{*}: K_{X_{n}} \cong K_{X}$. We have $K_{X_{n}} \sim\left(g_{2} \circ \cdots \circ g_{n}\right)^{*} K_{X_{1}}, K_{X_{n}} \cong K_{X}$ for all $n \in \boldsymbol{N}$ and $m K_{X_{1}} \sim \psi^{*} \mu^{*} L$ for a very ample line bundle $L$ on $S$. Hence $\left(g_{2} \circ \cdots \circ g_{n}\right)^{*}$ induces a linear 
automorphism of $H^{0}\left(X_{n}, m K_{X_{n}}\right)\left(=H^{0}\left(X_{1}, m K_{X_{1}}\right)\right)$ and $\mu \circ \psi \circ g_{2} \circ \cdots \circ g_{n}$ : $X_{n} \rightarrow S$ also gives the Iitaka fibration of $X_{n}$ by the same reason as in the proof of Proposition 2.5. Hence $\psi_{n}:=\psi \circ g_{2} \circ \cdots \circ g_{n}: X_{n} \rightarrow T$ also gives an equidimensional elliptic fibration over $T$ for all $n$.

Claim. The elliptic fibration $\psi: X_{1} \rightarrow T$ has at most multiple singular fibers of type ${ }_{m} I_{0}(m>1)$ generically in the codimension one discriminant locus $D$ of $T$. (cf. Definition $5.0(3)$ )

Proof. By the above remark, for all $n \in \boldsymbol{N}$, we have the following commutative diagram:

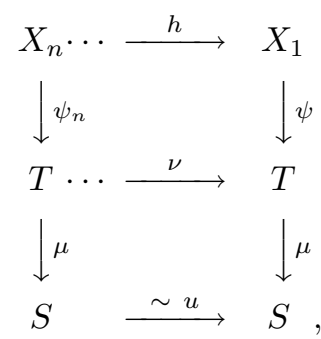

where $h: X_{n} \cdots \rightarrow X_{1}$ is a composite of flops, $G_{n}:=g_{2} \circ \cdots \circ g_{n}: X_{n} \rightarrow X_{1}$ is a finite étale covering, and $u: S \cong S$ (resp. $\nu: T \cdots \rightarrow T$ ) is an isomorphism (resp. a birational map). Since $\psi$ and $\psi_{n}$ are equidimensional and $h$ is an isomorphism in codimension one, for each prime divisor $\Gamma$ on $T$, its proper transforms by $\nu$ and $\nu^{-1}$ must be prime divisors. Since $\operatorname{dim}(T)=2, \nu$ is an isomorphism. (cf. [N1, Appendix A. Remark A.7]. Now assume the contrary. Then there exists a prime divisor $\Delta \subset D$ on $T$ such that for a general point $p \in \Delta$, all the irreducible components of $\psi^{-1}(p)$ are rational curves. Then by the same method as in the proof of Theorem 3.2, Remark (1) or Lemma 5.9, if we let $n \rightarrow \infty$, there exist infinitely many rational curves in the singular fibers of $\psi_{n}:=\psi \circ G_{n}: X_{n} \rightarrow T$ along a prime divisor $\Delta$ on $T$. Since a birational map $h$ is an isomorphism in codimension one, $\nu$ is an isomorphism, and $\psi$ is equidimensional, there exist infinitely many rational curves in the singular fibers of $\psi: X_{1} \rightarrow T$ along the prime divisor $\nu(\Delta)$ on $T$. This is a contradiction.

Proof of Theorem 5.1 continued. $\quad \psi: X_{1} \rightarrow T$ is equidimensional and $K_{X_{1}}$ is numerically $\psi$-trivial. Hence by Theorem $(\mathrm{N})$, a suitable finite étale covering $\tilde{X}_{1}$ of $X_{1}$ is isomorphic to the direct product $Z \times E$ of an elliptic curve $E$ and a nonsingular minimal algebraic surface $Z$ of general type. In particular, there exist no rational curves in fibers of $\psi . X$ is obtained from $X_{1}$ by a finite 
succession of flops. They are performed along rigid rational curves on $X_{1}$, which are $\psi$-horizontal. However, since $E$ acts on $\tilde{X}_{1} \cong Z \times E$ as translations over $Z$, the rational curves on $X_{1}$ are not rigid. Hence $X$ is isomorphic to $X_{1}$. Therefore we have the following diagram $X \stackrel{\psi}{\longrightarrow} T \stackrel{\mu}{\longrightarrow} S$ such that:

(1) $\psi: X \rightarrow T$ is a Seifert elliptic fibration,

(2) $\mu: T \rightarrow S$ is a birational morphism,

(3) $\varphi=\mu \circ \psi: X \rightarrow S$ is the Iitaka fibration of $X$.

Since $S$ is a normal surface with only log-terminal singularities, so is $T$. Hence $T$ is a normal surface with at most quotient singularities. Since $X$ and $Y$ are connected by flops, we have an isomorphism $X \cong Y$ by the same reason as above. Since $\mu$ is birational and $\psi$ is equidimensional, Proposition 2.5 and the rigidity lemma (cf. $[\mathrm{KM}]$ ) imply that $\psi \circ f \circ \psi^{-1}(t)$ is a point for all $t \in T$. Hence there exists $\lambda \in \operatorname{End}(T)$ such that $\lambda \circ \psi=\psi \circ f$. Since $\lambda$ is birational, it is an isomorphism by Zariski's main theorem. We have thus proved the theorem.

Example 1. Let $C$ be a nonsingular hyperelliptic curve of genus $g \geq 2$ with the hyperelliptic involution $\iota$. Let $E$ be a smooth elliptic curve and $t$ a translation of order 2 on $E$. Let $j:=(i, i, t)$ be the involution on $C \times C \times E$. Its action is free and the quotient $X:=C \times C \times E /\langle j\rangle$ is a smooth projective 3-fold with $\kappa(X)=2$. The projection $p: C \times C \times E \rightarrow C \times C$ induces on $X$ an elliptic fiber space structure $f: X \rightarrow C \times C /\langle i, i\rangle=: S$, where $S$ is a normal algebraic surface of general type with $(2 g+2)^{2} A_{1}$-singularities $Q_{i}\left(1 \leq i \leq(2 g+2)^{2}\right)$. At each $Q_{i}$, it has an isolated singular fiber whose support $E_{i}$ is isomorphic to a smooth elliptic curve $E /\langle t\rangle$. It is a principal fiber bundle outside them. There exist no rational curves on $X$ and $X$ is minimal. For an odd integer $n>1$, let [n] $: E \rightarrow E$ be multiplication by $n$. Then $\varphi:=\left(\operatorname{id}_{C}, \operatorname{id}_{C},[\mathrm{n}]\right) \in \operatorname{End}(C \times C \times E)$ commutes with the action of $j$ and induces a nontrivial surjective endomorphism $g$ of $X$ over $S$.

Next we treat the case where $\kappa(X)=\kappa(Y)=0$.

Theorem 5.4. Let $f: Y \rightarrow X$ be a nonisomorphic finite étale covering between minimal smooth projective 3 -folds $X$ and $Y$ which are birationally equivalent and $\kappa(X)=\kappa(Y)=0$. Then

(1) There exists an isomorphism between $X$ and $Y$, and $f$ can be regarded as a nontrivial surjective endomorphism of $X$.

(2) A suitable finite étale covering $\tilde{X}$ of $X$ is isomorphic to an abelian 3fold or the direct product $\tilde{T} \times E$ of an elliptic curve $E$ and a smooth $K 3$ surface $\tilde{T}$.

(3) In the latter case, $X$ has the structure of a Seifert elliptic (resp. K3 or 
Enriques) fiber space $\psi: X \rightarrow \tilde{T} / G=: W$ (resp. $\varphi: X \rightarrow E / G^{\prime}=: C$ ) over the qoutient space $W$ (resp. $C$ ) of $\tilde{T}$ (resp. E) by a finite group $G$ (resp. $\left.G^{\prime}\right)$. $f$ is compatible with these two fiber space structures and induces an automorphism $f^{\prime}$ of $W$ (resp. an endomorphism $h$ of $C$ ).

Proof. Since $\kappa(X)=0$ and $K_{X}$ is nef, it follows from the 3-dimensional abundance theorem due to [Mi1], [Mi2] and [Kaw2] that $K_{X}$ is a torsion element in $\operatorname{Pic}(X)$. In particular, we have $C_{1}(X)_{\mathbf{R}}=0$.

Claim. $\pi_{1}(X)$ is an infinite group.

Proof. Since $X$ and $Y$ are isomorphic in codimension 1, we have an isomorphism $\pi_{1}(X) \cong \pi_{1}(Y)$. Assume that $\pi_{1}(X)$ is a finite group. Then the injective homomorphism $f_{*}: \pi_{1}(Y) \rightarrow \pi_{1}(X)$ is an isomorphism. Hence we have $\operatorname{deg}(f)=\left[\pi_{1}(X): \pi_{1}(Y)\right]=1$ and $f$ is an isomorphism. This is a contradiction.

Hence by Bogomolov's decomposition theorem (cf. [Be]), a suitable finite étale covering $q: \tilde{X} \rightarrow X$ of $X$ is isomorphic to an abelian 3 -fold or the direct product of a smooth $K 3$ surface $\tilde{T}$ and an elliptic curve $E$. $Y$ can be obtained from $X$ by a finite succession of flops which are performed along rigid rational curves on $X$. If $\tilde{X}$ is isomorphic to an abelian 3-fold, $X$ contains no rational curves and $X \cong Y$. Next, we consider the case where $\tilde{X} \cong \tilde{T} \times E$, where $\tilde{T}$ is a smooth $K 3$ surface and $E$ is an elliptic curve. Note that any rational curve on $\tilde{X}$ is contained in fibers of the second projection $p_{2}: \tilde{X}:=\tilde{T} \times E \rightarrow E$. The first projection $p_{1}: \tilde{X}:=\tilde{T} \times E \rightarrow \tilde{T}$ is a trivial elliptic bundle over $\tilde{T}$ and $E$ acts on $\tilde{X}$ as translations over $\tilde{T}$. Therefore, the rational curves on $\tilde{X}$ (hence, on $X$ ) are not rigid and we have an isomorphism $X \cong Y$. By [Be, Proposition 3], we may assume that $q: \tilde{X}:=\tilde{T} \times E \rightarrow X$ is a minimal split covering. That is, $q$ is a finite Galois étale covering and the Galois group $\tilde{G}:=\operatorname{Gal}(\tilde{X} / X)$ does not contain an element of the form $\left(\operatorname{id}_{\tilde{T}}, \tau\right)$, where $0 \neq \tau \in E$ means a translation of the elliptic curve $E$. By the minimality of $q$, the composite map $f \circ q: \tilde{X} \stackrel{q}{\rightarrow} X \stackrel{f}{\rightarrow} X$ factors through $q: \tilde{X} \rightarrow X$. Hence there exists an endomorphism $\tilde{f}$ of $\tilde{X}$ such that $q \circ \tilde{f}=f \circ q$. By Corollary 2.13, there exist $v \in \operatorname{Aut}(\tilde{T})$ and $u \in \operatorname{End}(E)$ such that $\tilde{f}=v \times u: \tilde{X} \rightarrow \tilde{X}$. Again by [Be, Section 3, Lemma], every automorphism $g$ of $\tilde{X}$ can be uniquely decomposed as $g=g_{1} \times g_{2}$, where $g_{1} \in \operatorname{Aut}(\tilde{T}), g_{2} \in \operatorname{Aut}(E)$. Let $\tau: \operatorname{Aut}(\tilde{X}) \rightarrow \operatorname{Aut}(\tilde{T})$ (resp. $\rho: \operatorname{Aut}(\tilde{X}) \rightarrow \operatorname{Aut}(E)$ ) be a group homomorphism defined by $\tau(g)=g_{1}$ (resp. $\rho(g)=g_{2}$ ) and put $G:=\tau(\tilde{G})$ (resp. $G^{\prime}:=\rho(\tilde{G})$ ). The first (resp. the second) projection $p_{1}: \tilde{X} \rightarrow \tilde{T}$ (resp. $p_{2}: \tilde{X} \rightarrow E$ ) induces on $X$ a Seifert 
elliptic (resp. $K 3$ or Enriques) fiber space structure $\psi: X:=\tilde{T} \times E / \tilde{G} \rightarrow$ $\tilde{T} / G=: W\left(\right.$ resp. $X:=\tilde{T} \times E / \tilde{G} \rightarrow E / G^{\prime}=: C$ ) over the quotient $W$ (resp. $C$ ) of $\tilde{T}$ by a finite group $G$ (resp. $G^{\prime}$ ). Since $f \in \operatorname{End}(X)$ is induced by $\tilde{f}=v \times u \in$ $\operatorname{End}(\tilde{X})($ where $v \in \operatorname{Aut}(\tilde{T}), u \in \operatorname{End}(E))$ by Corollary 2.13 and $X \cong \tilde{X} / \tilde{G}, \tilde{f}$ is compatible with the action of $\tilde{G}$. Hence, $v$ (resp. $u$ ) is also compatible with the action of $G$ (resp. $G^{\prime}$ ) and induces an automorphism $v^{\prime}$ (resp. an endomorphism $u^{\prime}$ ) of the base space $W$ (resp. $C$ ).

We shall briefly discuss the following example.

Example 2. Let $S$ be a smooth $K 3$ surface with the fixed point free involution $\iota$ such that the quotient $T:=S /\langle\iota\rangle$ is an Enriques surface. Let $E$ be a smooth elliptic curve and $\tau$ the group inversion on $E$. Let $j:=(i, \tau)$ be the involution on $S \times E$. Its action is free and the quotient $Y:=S \times E /\langle j\rangle$ is a nonsingular minimal projective 3 -fold with $\kappa(Y)=0$. The first projection $p: S \times E \rightarrow S$ induces on $Y$ an elliptic fiber space structure $h: Y \rightarrow T$ which is a principal fiber bundle with typical fiber $E$. The second projection $q: S \times E \rightarrow E$ induces on $Y$ a Seifert $K 3$ fiber space structure $g: Y \rightarrow E /\langle\tau\rangle \cong \mathbf{P}^{1}$. It has 4 multiple fibers of multiplicity 2 whose supports are isomorphic to $T$. For any integer $n>1, \psi:=\left(\operatorname{id}_{S},[n]\right) \in \operatorname{End}(S \times E)$ commutes with the action of $j$ and induces a nontrivial surjective endomorphism $\mu$ of $Y$, which is compatible with the above two fiber space structures.

Finally we consider the case where $\kappa(X)=\kappa(Y)=1$. In order to prove the theorem, we prepare a few propositions.

Proposition 5.5. Let $f: Y \rightarrow X$ be a nonisomorphic finite étale covering between nonsingular projective $m$-folds $X$ and $Y$ which are birationally equivalent. Assume that $\kappa(X) \geq 0$. Let $X_{z}(z \in Z)$ be the 'general' fiber of the Iitaka fibration $\varphi: X \cdots \rightarrow Z$ of $X$ and $F$ be a nonsingular model of $X_{z}$. Then we have $\chi\left(\mathscr{O}_{F}\right)=0$.

Proof. Let $\varphi^{\prime}: X^{\prime} \rightarrow Z^{\prime}$ be a nonsingular model of $\varphi: X \cdots \rightarrow Z$. We have the following commutative diagram:

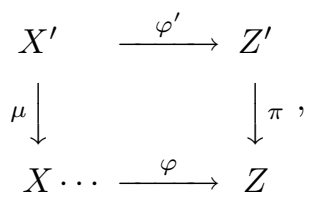

where $X^{\prime}$ and $Z^{\prime}$ are nonsingular, $\mu$ and $\pi$ are birational morphisms and $\varphi^{\prime}$ is a morphism. Then $Y^{\prime}:=Y \times_{X} X^{\prime}$ is nonsingular and birationally equivalent 
to $Y$ and the natural map $f^{\prime}: Y^{\prime} \rightarrow X^{\prime}$ is a finite étale covering. By the same argument as in the proof of Proposition 2.5, $f^{\prime \prime}:=\varphi^{\prime} \circ f^{\prime}: Y^{\prime} \rightarrow Z^{\prime}$ is also a nonsingular model of the Iitaka fibration of $X$. Hence we have $\chi\left(\mathscr{O}_{Y_{p}^{\prime}}\right)=\chi\left(\mathscr{O}_{X_{p}^{\prime}}\right)$ for 'general' $p \in Z^{\prime}$. On the other hand, since $f^{\prime}$ is finite étale, we have $\chi\left(\mathscr{O}_{Y_{p}^{\prime}}\right)=\operatorname{deg}\left(f^{\prime}\right) \times \chi\left(\mathscr{O}_{X_{p}^{\prime}}\right)$. Since $\operatorname{deg}\left(f^{\prime}\right)>1$, we have $\chi\left(\mathscr{O}_{X_{p}^{\prime}}\right)=0$ and this implies the claim.

Corollary 5.6. $\quad$ Let $X$ be a smooth projective $m$-fold with $\kappa(X) \geq 0$ which admits a nontrivial surjective endomorphism. Then the same results as in Proposition 5.5 hold for $X$.

Proposition 5.7. Let $f: Y \rightarrow X$ be a nonisomorphic finite étale covering between nonsingular minimal projective 3 -folds $X$ and $Y$ which are birationally equivalent and $\kappa(X)=1$. Then the 'general' fiber $X_{c}(c \in C)$ of the Iitaka fibration $\varphi: X \rightarrow C$ is isomorphic to either an abelian surface or a hyperelliptic surface.

Proof. Since $K_{Y}$ is nef, the 'general' fiber $X_{c}(c \in C)$ of $\varphi$ is also a minimal algebraic surface with $\kappa=0$. Hence Proposition 5.5 and the classification theory of algebraic surfaces imply the claim.

Now we recall the following Fact:

Fact. Let $S$ be a hyperelliptic surface.

(1) Then $S$ has only 2 types of nonisomorphic fibrations:

Type (A). The Albanese map $p_{S}: S \rightarrow \operatorname{Alb}(S)$ gives on $S$ the structure of an elliptic fiber bundle over an elliptic curve $\operatorname{Alb}(S)$.

Type(B). $E:=\operatorname{Aut}^{0}(S)$ is an elliptic curve and the natural projection $q_{S}: S \rightarrow C_{S}:=S / E$ is a Seifert elliptic surface over the quotient $C_{S} \cong \mathbf{P}^{1}$. (cf. Proposition 2.8)

(2) Let $F_{S}$ (resp. $G_{S}$ ) be the general fiber of $p_{S}$ (resp. $\left.q_{S}\right)$. Then we have $\rho(S)=2$ and $\overline{N E}(S)=\mathbf{R}_{\geq 0}\left[F_{S}\right]+\mathbf{R}_{\geq 0}\left[G_{S}\right]$.

Proposition 5.8. Let $f: S \rightarrow T$ be a surjective morphism between hyperelliptic surfaces $S$ and $T$. Then there exists a morphism $u: \operatorname{Alb}(S) \rightarrow$ $\operatorname{Alb}(T)$ (resp. $\left.h: C_{S} \rightarrow C_{T}\right)$ such that $p_{T} \circ f=u \circ p_{S}\left(\right.$ resp. $\left.q_{T} \circ f=h \circ q_{S}\right)$.

Proof. By the universality of the Albanese map, there exists an affine morphism $u: \operatorname{Alb}(S) \rightarrow \operatorname{Alb}(T)$ with $p_{T} \circ f=u \circ p_{S}$. Since $\rho(S)=\rho(T)=2$, $f$ is a finite morphism by Lemma 2.3. By Proposition 4.2 and the above Fact, there is a one-to-one correspondence between the edges of $\overline{N E}(S)$ and the edges 
of $\overline{N E}(T)$ under the isomorphisms $f^{*}$ and $f_{*}$. Since $f_{*}\left[F_{S}\right] \in \mathbf{R}_{\geq 0}\left[F_{T}\right]$ and $f^{*}\left[F_{T}\right] \in \mathbf{R}_{\geq 0}\left[F_{S}\right]$, we have $f_{*}\left[G_{S}\right] \in \mathbf{R}_{\geq 0}\left[G_{T}\right]$ and $f^{*}\left[G_{T}\right] \in \mathbf{R}_{\geq 0}\left[G_{S}\right]$. Hence there exists a morphism $h: C_{S} \rightarrow C_{T}$ such that $q_{T} \circ f=h \circ q_{S}$.

The following lemma is a key step towards the proof of Theorem 5.10.

Lemma 5.9. Let $f: Y \rightarrow X$ be a nonisomorphic finite étale covering between minimal smooth projective 3 -folds $X$ and $Y$ which are birationally equivalent and $\kappa(X)=1$. Let $\varphi: X \rightarrow C$ be the Iitaka fibration of $X$. Then a nonsingular model of each irreducible component of the singular fibers of $\varphi$ is not a rational surface.

Proof. $X$ and $Y$ are isomorphic in codimension one and connected by a finite succession of flops. Hence, by the same argument as in the proof of Theorem 5.1, $f: Y \rightarrow X$ induces an infinite tower of nonisomorphic finite étale coverings: $X \stackrel{f}{\longleftarrow} Y \stackrel{f_{1}}{\longleftarrow} Y_{1} \stackrel{f_{2}}{\longleftarrow} \cdots \stackrel{f_{n}}{\longleftarrow} Y_{n} \leftarrow \cdots$ between minimal nonsingular projective 3 -folds $Y_{n}$ 's which are all connected to $X$ by a finite sequence of flops. And we have the following commutative diagram:

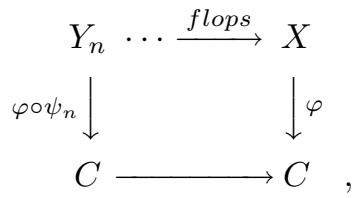

where we put $\psi_{n}:=f \circ f_{1} \circ \cdots \circ f_{n}$. Now assume the contrary. Let $S$ be an irreducible component of the singular fiber $\varphi^{-1}(p)(p \in C)$ whose nonsingular model $u: S^{\prime} \rightarrow S$ is a rational surface. If we put $D:=\psi_{n}^{-1}(S) \underset{S}{\times} S^{\prime}$, the second projection $q: D \rightarrow S^{\prime}$ is a finite étale covering. Since $S^{\prime}$ is simply connected, $D$ consists of $d_{n}:=\operatorname{deg}\left(\psi_{n}\right)>1$ disjoint connected components. Since the first projection $p: D \rightarrow \psi_{n}^{-1}(S)$ is a birational morphism, $\psi_{n}^{-1}(S)$ also consists of $d_{n}$ irreducible components. The above diagram implies that there exist $d_{n}$ irreducible components in the singular fibers of $\varphi: X \rightarrow C$. Since we have $d_{n} \rightarrow \infty$ as $n \rightarrow \infty$, this is a contradiction.

Now we are ready to state MAIN THEOREM in the case of $\kappa=1$.

Theorem 5.10. Let $f: Y \rightarrow X$ be a nonisomorphic finite étale covering between minimal nonsingular projective 3 -folds $X$ and $Y$ which are birationally equivalent and $\kappa(X)=1$. Assume that the 'general' fiber $X_{c}(c \in C)$ of the Iitaka fibration $\varphi: X \rightarrow C$ is a hyperelliptic surface. Then 
(1) There exists an isomorphism between $X$ and $Y$, and $f$ can be regarded as a nontrivial surjective endomorphism of $X$.

(2) We have the diagram of fiber spaces $X \stackrel{\pi}{\longrightarrow} T \stackrel{q}{\longrightarrow} C$, where $\pi: X \rightarrow T$ is a Seifert elliptic fiber space over a normal surface $T$ with at most quotient singularities, $q: T \rightarrow C$ is a $\mathbf{P}^{1}$-fiber space and $\varphi=q \circ \pi$.

(3) $f \in \operatorname{End}(X)$ induces an endomorphism (resp. an automorphism) of $T$ (resp. $C$ ) and is compatible with the above diagram.

(4) A suitable finite étale covering $\tilde{X}$ of $X$ is isomorphic to the direct product $\tilde{T} \times E$ of an elliptic curve $E$ and a nonsingular minimal algebraic surface $\tilde{T}$ with $\kappa(\tilde{T})=1$.

Proof. Here we use Fujiki's theory of 'relative generic quotient' [F2], [F3], which are stated in the category of compact analytic spaces in the class $\mathscr{C}$. However, if we replace Douady spaces by Hilbert schemes, his method also works in the category of projective schemes. Let $U \subset C$ be a Zariski open subset over which $\varphi$ is smooth. $\operatorname{Aut}_{U}^{0} X_{U}$, which is the relative automorphism group

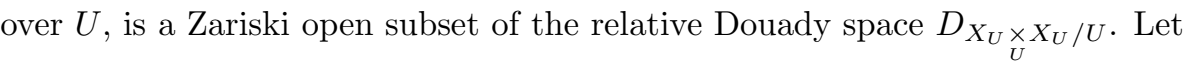
$G^{*}:=\operatorname{Aut}_{C}^{*} X$ be the essential closure of $\operatorname{Aut}_{U}^{0} X_{U}$ in $D_{X \times C}{ }_{C}$, , i.e. the union of those irreducible components of the closure which are mapped surjectively onto $C$. By Fujiki [F1], $G^{*}$ is analytic and compact. Let $S:=X / G^{*}$ be the relative generic quotient of $X$ by $G^{*}$ in the sense of [F2], [F3]. Since the 'general' fiber of $\varphi$ is a hyperelliptic surface, $G_{y}:=G_{y}^{*} \cap \mathrm{Aut}^{0} X_{y}$ is an elliptic curve for 'general' $y \in U, p: S \rightarrow C$ is a $\mathbf{P}^{1}$-fiber space and the general fiber of $\pi^{\prime}: X \cdots \rightarrow S$ is an elliptic curve. By the elimination of the indeterminancy of $\pi^{\prime}$, we can take a blow-up $\mu: \hat{X} \rightarrow X$ of $X$ such that $g:=\pi^{\prime} \circ \mu: \hat{X} \rightarrow S$ is a morphism and gives an elliptic fibration over $S$. Apply the minimal model program (MMP) (cf. [KM]) to $g: \hat{X} \rightarrow S$, we have an elliptic fibration $h: X^{\prime} \rightarrow S$ such that:

(1) it is birationally equivalent to $g: \hat{X} \rightarrow S$,

(2) $X^{\prime}$ has only $\mathbf{Q}$-factorial terminal singularities,

(3) $K_{X^{\prime}}$ is $h$-nef.

By the equidimensional model theorem by Nakayama [N1, Appendix A, Proposition A.6], if we perform a finite succesion of flops to $X^{\prime}$ and take a suitable birational morphism $u: W \rightarrow S$, we have an elliptic fibration $h^{\prime}$ : $X^{\prime \prime} \rightarrow W$ such that:

(1) $h$ and $u \circ h^{\prime}$ are birationally equivalent,

(2) $h^{\prime}$ is equidimensional,

(3) $X^{\prime \prime}$ has only Q-factorial terminal singularities,

(4) there exists an effective Q-divisor $\Lambda$ on $W$ such that $(W, \Lambda)$ is logterminal and $K_{X^{\prime \prime}} \sim_{Q} h^{\prime *}\left(K_{W}+\Lambda\right)$. In particular, $K_{X^{\prime \prime}}$ is numerically $h^{\prime}$ - 
trivial.

Note that $K_{X^{\prime \prime}}$ is nef if and only if $K_{W}+\Lambda$ is nef on $W$. By [G2, Theorem 2.2 and Corollary 2.8], there exists a birational morphism $u^{\prime}: W \rightarrow T$ and an elliptic fibration $\pi: Z \rightarrow T$ over $T$ such that:

(1) $u^{\prime} \circ h^{\prime}$ and $\pi$ are birationally equivalent,

(2) $\pi$ is equidimensional,

(3) $Z$ has only Q-factorial terminal singularities,

(4) there exists an effective $\mathbf{Q}$-divisor $\Gamma$ on $T$ such that $(T, \Gamma)$ is log-terminal and $K_{Z} \sim \pi^{*}\left(K_{T}+\Gamma\right)$,

(5) $K_{Z}$ is nef. (i.e. $Z$ is the absolute minimal model of $X$ ).

$Z$ and $X$ are connected by a finite sequence of flops by [Kaw1] and [Kol1] and hence $Z$ is also nonsingular by [Kol1]. By completely the same method as in the proof of Theorem 5.1, the original $f: Y \rightarrow X$ induces an infinite tower of nonisomorphic finite étale coverings $X \sim Z \stackrel{g_{1}}{\longleftarrow} Z_{1} \stackrel{g_{2}}{\longleftarrow} Z_{2} \leftarrow \cdots \stackrel{g_{n}}{\longleftarrow} Z_{n} \leftarrow$ $\cdots$, between minimal nonsingular projective 3-folds $Z_{n}$ 's, where $\kappa\left(Z_{n}\right)=1$ and $Z_{n}$ is a minimal model of $X$ for all $n$. They are all connected to $X$ by a finite sequence of flops. Let $\varphi^{\prime}: Z \rightarrow C$ be the Iitaka fibration of $Z$. Its general fiber is also a hyperelliptic surface and it is decomposed as $\varphi^{\prime}=q \circ \pi$, where $q: T \rightarrow C$ is a $\mathbf{P}^{1}$-fiber space.

In fact, we have $\left(K_{Z}, F\right)=0$ for a genral fiber $F$ of $\pi$. Since $K_{Z}$ is $\mathbf{Q}$ linearly equivalent to the pullback of an ample $\mathbf{Q}$-divisor on $C, F$ is contracted to a point by $\varphi^{\prime}$. Hence the rigidity lemma implies the claim. We have the following commutative diagram:

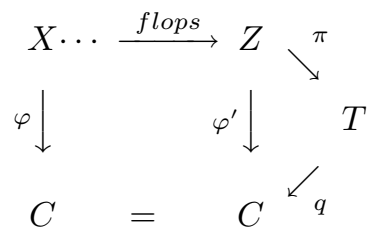

And the composite map $\varphi_{n}^{\prime}:=\varphi \circ g_{1} \circ \cdots \circ g_{n}: Z_{n} \rightarrow C$ also gives the Iitaka fibration of $Z_{n}$.

Claim. The elliptic fibration $\pi: Z \rightarrow T$ has at most multiple singular fibers of type ${ }_{m} I_{0}(m>1)$ generically along the codimension one discriminant locus $D$ of $T$. (cf. Definition 5.0.(3))

Proof. Assume the contrary. Then there exists some prime divisor $\Delta(\subset$ $D)$ on $T$ such that for a general point $p \in \Delta$, all the irreducible components of $\psi^{-1}(p)$ are rational curves. Note that the general fiber of $\varphi^{\prime}$ is a hyperelliptic 
surface and contains no rational curves. Hence $\Delta$ is contained in a fiber of $q: T \rightarrow C$, which is a $\mathbf{P}^{1}$-fiber space. Thus $\Delta$ is a rational curve and the nonsingular model of $\pi^{-1}(\Delta)$ is a rational surface. This contradicts Lemma 5.9 , hence the claim follows.

Proof of Theorem 5.10 continued. The elliptic fibration $\pi: Z \rightarrow T$ is equidimensional and $K_{Z}$ is numerically $\pi$-trivial. Hence by Theorem $(\mathrm{N})$, a suitable finite étale covering $\tilde{Z}$ of $Z$ is isomorphic to the direct product $\tilde{T} \times E$ of an elliptic curve $E$ and a nonsingular minimal algebraic surface $\tilde{T}$ with $\kappa(\tilde{T})=1$. There exist no rational curves in fibers of $\pi$. Hence, by completely the same method as in the proof of Theorem $5.1, X$ is isomorphic to $Z$ and we have the following diagram $X \stackrel{\pi}{\longrightarrow} T \stackrel{q}{\longrightarrow} C$ such that:

(1) $\pi: X \rightarrow T$ is a Seifert elliptic fiber space over a normal surface $T$ with at most quotient singularities,

(2) $q: T \rightarrow C$ is a $\mathbf{P}^{1}$-fiber space,

(3) the composite map $\varphi:=q \circ \pi$ gives the Iitaka fibration of $X$ and the 'general' fiber of $\varphi$ is a hyperelliptic surface.

Since $X$ and $Y$ are connected by flops, we have an isomorphism $X \cong$ $Y$ by the same reason as above. By Propositions 2.5 and 5.8, $f$ induces an automorphism (resp. an endomorphism) of $C$ (resp. $T$ ) and is compatible with the above diagram.

Example 3. Let $f: S \rightarrow C$ be a relatively minimal elliptic surface with a global section $o$ such that $\kappa(S)=1$ and $\chi\left(O_{S}\right) \neq 0$. $S$ can be considered as an elliptic curve $\mathscr{E}$ over the function field $K$ of $C$ with the origin $o$. Let $i: S \cdots \rightarrow S$ be a birational map of $S$ induced from the group inversion of $\mathscr{E}$ over $K$. Since $S$ is minimal, it is an automorphism of $S$. Let $E$ be a smooth elliptic curve and $t$ a translation of order 2 on $E$. Then $j:=(i, t) \in \operatorname{Aut}(S \times E)$ is a free involution and the quotient $X:=S \times E /\langle j\rangle$ is a smooth 3-fold with $\kappa(X)=1$. Let $n>1$ be an odd integer and $[n]: E \rightarrow E$ multiplication by $n$. Then $\psi:=\left(\operatorname{id}_{S},[n]\right)$, which is a surjective endomorphism of $S \times E$, induces a nontrivial surjective endomorphism $\varphi$ of $X$. The projection $S \times E \rightarrow C$, which is invariant by $\langle j\rangle$, induces on $X$ the Iitaka fibration $\phi: X \rightarrow C$ whose 'general' fiber is a hyperelliptic surface. The natural projection $S \times E \rightarrow S \rightarrow C$ induces the commutative diagram: $X \stackrel{g}{\longrightarrow} T:=S /\langle\iota\rangle \stackrel{p}{\longrightarrow} C$, where $g: X \rightarrow T$ is a Seifert elliptic fiber space, $p: T \rightarrow C$ is a $\mathbf{P}^{1}$-fiber space and $\phi=p \circ g$.

Furthermore, the natural projection $S \times E \stackrel{\left(f, \text { id }_{E}\right)}{\longrightarrow} C \times E \rightarrow C$ induces the commutative diagram $X \stackrel{h}{\longrightarrow} C \times E^{\prime} \stackrel{q}{\longrightarrow} C$, where $E^{\prime}:=E /\langle t\rangle$ is a smooth elliptic curve and a relative Albanese map $h: X \rightarrow C \times E^{\prime}$ of $\phi: X \rightarrow C$ is an elliptic fiber space with variable moduli in general, and $\phi=q \circ h$. 
The following lemma will be used in Section 6 to prove MAIN THEOREM (A).

Lemma 5.11. Let $f: S \rightarrow S$ be a nontrivial étale endomorphism of a relatively minimal elliptic ruled surface $S$. Then $f$ preserves the elliptic fiber space structure $\varphi: S \rightarrow \mathbf{P}^{1}$ and induces an automorphism of the base curve $\mathbf{P}^{1}$.

Proof. The proof of this lemma will be done in a similar way as that of Theorem 5.4. First we recall the following Fact.

Fact. Let $S$ be a relatively minimal elliptic ruled surface.

(1) Then $S$ has exactly 2 types of nonisomorphic fiber spaces structures:

Type (a). The Albanese map $\alpha: S \rightarrow \operatorname{Alb}(S)=: E$ gives on $S$ a $\mathbf{P}^{1}$-bundle structure over the elliptic curve $E$.

Type (b). $\quad S$ has the structure of a Seifert elliptic surface $\varphi: S \rightarrow \mathbf{P}^{1}$ and a suitable finite Galois étale covering $q: \tilde{S} \rightarrow S$ of $S$ is isomorphic to the direct product $\mathbf{P}^{1} \times E$ of $\mathbf{P}^{1}$ and an elliptic curve $E$. Moreover, the Galois group $G:=\operatorname{Gal}(\tilde{S} / S)$ does not contain an element of the form $\left(\operatorname{id}_{P^{1}}, \tau\right)$, where $0 \neq \tau \in E$ means a translation of the elliptic curve $E$. (cf. Proof of Theorem $5.4)$

(2) We have $\rho(S)=2$ and $\overline{N E}(S)=\mathbf{R}_{\geq 0}[F]+\mathbf{R}_{\geq 0}[G]$, where $F$ (resp. $G$ ) is a 'general' fiber of $\alpha$ (resp. $\varphi$ ).

From the universality of the Albanese map, there exists $u \in \operatorname{End}(E)$ such that $\alpha \circ f=u \circ \alpha$. By Proposition 4.2 and the above Fact, $f^{*}$ and $f_{*}$ give a permutation of the edges of $\overline{N E}(S)$. Since $f_{*}[F] \in \mathbf{R}_{\geq 0}[F]$ and $f^{*}[F] \in \mathbf{R}_{\geq 0}[F]$, we have $f_{*}[G] \in \mathbf{R}_{\geq 0}[G]$ and $f^{*}[G] \in \mathbf{R}_{\geq 0}[G]$. Hence $f$ preserves the elliptic surface structure $\varphi: S \rightarrow \mathbf{P}^{1}$. By [Be, Proposition 3], the composite map $f \circ q: \tilde{S} \rightarrow S$ factors through $q: \tilde{S} \rightarrow S$ and there exists an étale endomorphism $\tilde{f}$ of $\tilde{S}$ such that $q \circ \tilde{f}=f \circ q$. Hence by Proposition 2.12, $\tilde{f}$ can be decomposed as $\tilde{f}=g \times h$, where $g \in \operatorname{Aut}\left(\mathbf{P}^{1}\right)$ and $h \in \operatorname{End}(E)$, since $\mathbf{P}^{1}$ is simply connected. If we take a quotient of the first projection $p: S \cong \mathbf{P}^{1} \times E \rightarrow \mathbf{P}^{1}$ by the finite group $G$, we obtain the original elliptic fibration $\varphi: S \rightarrow \mathbf{P}^{1}$. Hence by the same method as in the proof of Theorem 5.4, $f$ induces an automorphism of the base curve $\mathbf{P}^{1}$ of $\varphi: S \rightarrow \mathbf{P}^{1}$.

\section{§6. Proof of MAIN THEOREM (A)}

In this section, we shall prove MAIN THEOREM (A).

6.1. First, we shall consider the case (1) where $\kappa(X)=2$. The following proposition plays a key role in this section. 
Proposition 6.1.1. Let $f: X \rightarrow T$ be a Seifert elliptic fiber space from a smooth projective $n$-fold $X$ of dimension $\geq 3$ to a normal projective variety $T$ and $u: X^{\prime} \rightarrow X$ the blowing-up of $X$ along a smooth elliptic curve $F$ which is a fiber of $f$. Then

(1) $X^{\prime}$ also has the Seifert elliptic fiber space structure $g: X^{\prime} \rightarrow T^{\prime}$ over a normal variety $T^{\prime}$ and we have the following commutative diagram of fiber spaces:

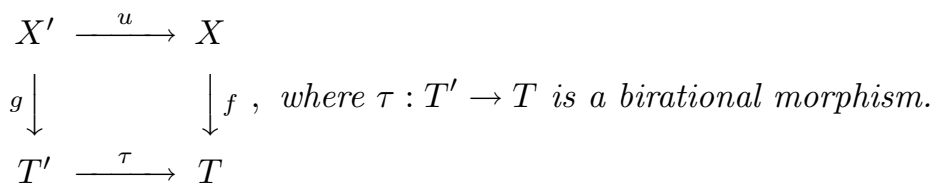

(2) A suitable finite étale covering $\tilde{X}^{\prime}$ of $X^{\prime}$ is isomorphic to the direct product $\tilde{T}^{\prime} \times E$ of an elliptic curve $E$ and a smooth projective variety $\tilde{T}^{\prime}$.

Proof. By Theorem $[\mathrm{N}]$ in Section 5, a suitable finite Galois étale covering $\varphi: \tilde{X} \rightarrow X$ of $X$ is isomorphic to the direct product $\tilde{T} \times E$ of an elliptic curve $E$ and a nonsingular projective variety $\tilde{T}$. Then $Y:=\tilde{X} \underset{X}{\times} X^{\prime}$ is nonsingular, the first projection $\tilde{u}: Y \rightarrow \tilde{X}$ is a birational morphism and the second projection $\tilde{\varphi}: Y \rightarrow X^{\prime}$ is a finite étale covering. $\tilde{u}$ is an isomorphism over $X$ outside $F$ and $\varphi^{-1}(F)$ is a disjoint union of smooth elliptic curves contained in fibers of the first projection $p: \tilde{X} \cong \tilde{T} \times E \rightarrow \tilde{T}$. Let $D$ be the exceptional divisor of u. $\left.u\right|_{D}: D \rightarrow F$ is a $\mathbf{P}^{n-2}$-bundle over $F$ and $K_{X^{\prime}} \sim u^{*} K_{X}+(n-2) D$. Since $\tilde{\varphi}$ and $\varphi$ are finite étale, we have $K_{Y} \sim \tilde{\varphi}^{*} K_{X^{\prime}} \sim \tilde{u}^{*} K_{\tilde{X}}+(n-2) \tilde{\varphi}^{*} D$. Let $\tilde{F}$ be an arbitrary connected component of $\varphi^{-1}(F)$, which is a smooth elliptic curve. Since each fiber of $\tilde{u}$ is connected by Zariski's connectedness theorem, $\tilde{D}:=\tilde{u}^{-1}(\tilde{F})$ is also a connected component of $\tilde{\varphi}^{-1}(D)$. Since $\tilde{\varphi}$ is finite étale and $\mathbf{P}^{n-2}$ is simply connected, $\tilde{u}: \tilde{D} \rightarrow \tilde{F}$ is also a $\mathbf{P}^{n-2}$-bundle. Since the restriction of $\tilde{\varphi}$ to $\tilde{u}^{-1}(x)\left(\cong \mathbf{P}^{n-2}\right)$ is an isomorphism for each $x \in \tilde{F}$, the projection formula implies that $\tilde{D}\left|\tilde{u}^{-1}(x) \cong D\right| \tilde{\varphi}_{*} \tilde{u}^{-1}(x) \cong \mathscr{O}_{P^{n-2}}(-1)$. Hence $\tilde{u}: Y \rightarrow \tilde{X}$ is the blow-up of $\tilde{X}$ along $\varphi^{-1}(F)$ by Nakano-Fujiki's contraction theorem. Since all the irreducible components of $\varphi^{-1}(F)$ are contained in fibers of $p$, we have an isomorphism $Y \cong \tilde{T}^{\prime} \times E$, where $\tilde{T}^{\prime}$ is obtained by blowing-up $\tilde{T}$ at finite number of points $p\left(\varphi^{-1}(F)\right)$. By construction, the Galois group $G:=\operatorname{Gal}\left(Y / X^{\prime}\right)$ induces a finite automorphism group $H$ of $\tilde{T}^{\prime}$ and the first projection $p^{\prime}: Y \rightarrow \tilde{T}^{\prime}$ induces a morphism $g: X^{\prime}=Y / G \rightarrow \tilde{T}^{\prime} / H=: T^{\prime}$, which gives $X^{\prime}$ the Seifert elliptic fiber space structure over $T^{\prime}$ and the proof is finished.

Proof of MAIN THEOREM (A). Case (1): $\kappa(X)=2$. 
Let $f: X \rightarrow X$ be a nontrivial surjective endomorphism of a smooth projective 3-fold $X$ with $\kappa(X)=2$. By taking a suitable power of $f$, we have the following commutative diagram:

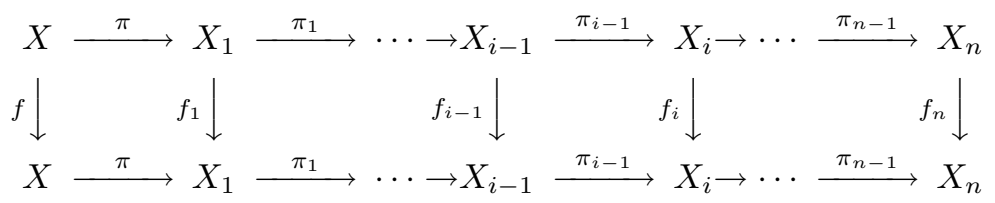

where

(1) $f_{i}$ is a nontrivial surjective endomorphism of $X_{i}$,

(2) $\pi_{i-1}: X_{i-1} \rightarrow X_{i}$ is (the inverse of) the blow-up along a smooth elliptic curve $C_{i}$ on $X_{i}$ such that $f_{i}^{-1}\left(C_{i}\right)=C_{i}$,

(3) $X_{n}$ is the unique nonsingular minimal model of $X$ (cf. Theorem 5.1) and $f_{n}$ is then a minimal reduction of $f$.

MAIN THEOREM (A), Case (1) immediately follows from the following assertions.

Assertions. For all $0 \leq i \leq n$, (here we put $X_{0}:=X$ )

$(1)_{i} X_{i}$ has the structure of a Seifert elliptic fiber space $\varphi_{i}: X_{i} \rightarrow T_{i}$ over a normal surface $T_{i}$ with at most quotient singularities. And a suitable finite étale covering $\tilde{X}_{i}$ of $X_{i}$ is isomorphic to the direct product $\tilde{T}_{i} \times E$ of an elliptic curve $E$ and a nonsingular algebraic surface $\tilde{T}_{i}$ of general type.

$(2)_{i}$ There exists an automorphism $\rho_{i}$ of $T_{i}$ such that $\rho_{i} \circ \varphi_{i}=\varphi_{i} \circ f_{i}$.

$(3)_{i} C_{i}$ is contained in fibers of $\varphi_{i}$.

Proof. The proof is done by a descending induction on $i$. For $i=n$, $(1)_{n}$ and $(2)_{n}$ are clear from Theorem 5.1. To show $(3)_{n}$, assume the contrary. Then $D_{n}:=\varphi_{n}\left(C_{n}\right)$ is an irreducible curve on $T_{n}$ and let $S_{n}:=\varphi_{n}^{-1}\left(D_{n}\right)$ be a Seifert elliptic surface over $D_{n}$. $S_{n}$ contains $C_{n}$ and may be singular. Since $C_{n}=f_{n}^{-1}\left(C_{n}\right) \subset f_{n}^{-1}\left(S_{n}\right)=\varphi_{n}^{-1} \circ \rho_{n}^{-1}\left(D_{n}\right), D_{n}$ is contained in $\rho_{n}^{-1}\left(D_{n}\right)$. Since $\rho_{n} \in \operatorname{Aut}\left(T_{n}\right), \rho_{n}^{-1}\left(D_{n}\right)$ is irreducible. Hence we have $D_{n}=\rho_{n}^{-1}\left(D_{n}\right)$ and $f_{n}^{-1}\left(S_{n}\right)=\varphi_{n}^{-1}\left(D_{n}\right)=S_{n}$. Let $F$ be a general fiber of $\left.\varphi_{n}\right|_{S_{n}}: S_{n} \rightarrow D_{n}$ and let $m:=\left(C_{n}, F\right)>0$ be the mapping degree of $C_{n}$. Since $f_{n}$ is a nonisomorphic finite étale covering of $d:=\operatorname{deg}\left(f_{n}\right)>1, f_{n}^{-1}\left(C_{n} \cap F\right)=f_{n}^{-1}\left(C_{n}\right) \cap f_{n}^{-1}(F)=$ $C_{n} \cap f_{n}^{-1}(F)$ consists of $\operatorname{dm}(>m)$ distinct points. On the other hand, $f_{n}^{-1}(F)$ is also a general fiber of $\varphi_{n}: S_{n} \rightarrow T_{n}$. This is a contradiction and $(3)_{n}$ is proved.

Next, suppose that the assertions hold true for $i+1$. Then Proposition 6.1.1 implies $(1)_{i}$ easily and we have the following commutative diagram: 


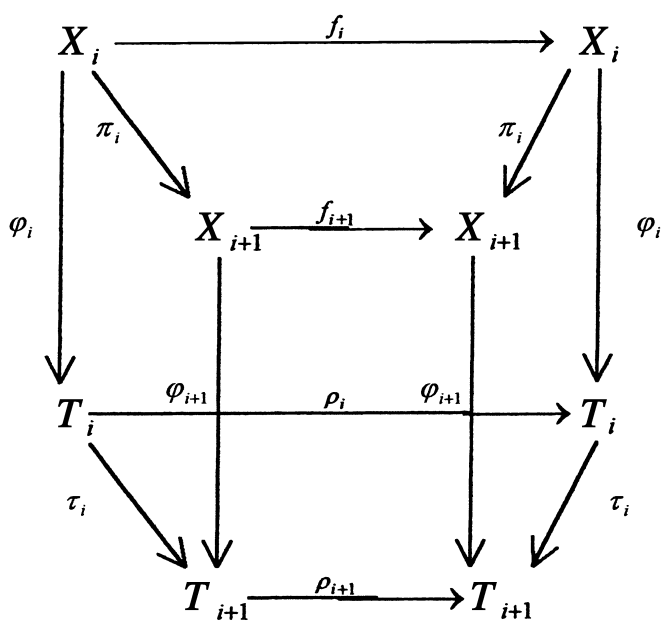

Since $\pi_{i}$ and $\tau_{i}$ are birational, $f_{i+1}^{-1}\left(C_{i+1}\right)=C_{i+1}$, and $\varphi_{i}$ is equidimensional, $f_{i}$ induces a surjective endomorphism $\rho_{i}$ of $T_{i}$ such that $\rho_{i} \circ \varphi_{i}=\varphi_{i} \circ f_{i}$ and $\tau_{i} \circ \rho_{i}=\rho_{i+1} \circ \tau_{i}$ by the rigidity lemma. Since $\rho_{i}$ is a finite birational morphism by $(2)_{i}$ and Lemma 2.3 , it is an isomorphism by Zariski's main theorem. Thus $(2)_{i}$ is proved. $(3)_{i}$ is derived from $(2)_{i}$ and the property that $f_{i}^{-1}\left(C_{i}\right)=C_{i}$ by the same argument as in $(3)_{n}$.

Example 6.1. Under the same situation as in Example 1 in Section 5, let $\pi: X^{\prime} \rightarrow X$ be the blowing-up of $X$ along the isolated singular fibers $E_{i}$ 's $\left(1 \leq i \leq(2 g+2)^{2}\right)$. Let $u: T \rightarrow C \times C$ be the blowing-up at the $(2 g+2)^{2}$ fixed points by the action of $(i, i) \in \operatorname{Aut}(C \times C) . j \in \operatorname{Aut}(C \times C \times E)$ can be extended to $j^{\prime} \in \operatorname{Aut}(T \times E)$ which acts freely on $T \times E$. $X^{\prime}$ is isomorphic to the quotient of $T \times E /\left\langle j^{\prime}\right\rangle$ and we have the commutative diagram below, where $\lambda: S^{\prime} \rightarrow S$ is the minimal resolution of $S . f^{\prime}: X^{\prime} \rightarrow S^{\prime}$ is a Seifert elliptic fiber space and has multiple fibers of type ${ }_{2} I_{0}$ along $(-2)$-curves $D_{j}$ 's $\left(1 \leq j \leq(2 g+2)^{2}\right)$ such that $\lambda\left(D_{j}\right)=Q_{j} . g \in \operatorname{End}(X)$ can be naturally extended to $g^{\prime} \in \operatorname{End}\left(X^{\prime}\right)$, which preserves the elliptic fiber space structure $f^{\prime}: X^{\prime} \rightarrow S^{\prime}$.

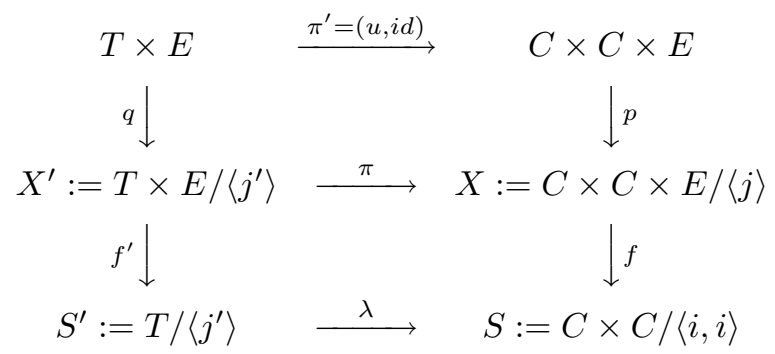


6.2. Next, we shall consider the case $(3)$ where $\kappa(X)=0$. As a first step, we consider the following situation.

Proposition 6.2.1. Let $f: V \rightarrow V$ be a nontrivial surjective endomorphism of a smooth projective 3 -fold $V$ which is birational but not isomorphic to an abelian 3 -fold. Then

(1) $V$ has the structure of an elliptic bundle $p: V \rightarrow S$ (i.e. a principal fiber bundle whose typical fiber and structure group are elliptic curves) over a smooth algebraic surface $S$ which is birational to an abelian surface.

(2) $f$ is compatible with $p$ and induces an automorphism $g$ of $S$.

(3) A suitable finite étale covering $\tilde{V}$ of $V$ is isomorphic to the direct product $S^{\prime} \times E$ of an elliptic curve $E$ and a smooth algebraic surface $S^{\prime}$ which is birational to an abelian surface.

Proof. By taking a suitable power of $f$, let $f_{n}: V_{n} \rightarrow V_{n}$ be the minimal reduction of $f: V \rightarrow V$. Since $V_{n}$ is minimal and birational to an abelian 3 -fold, it is isomorphic to an abelian 3-fold.

We have the following commutative diagram:

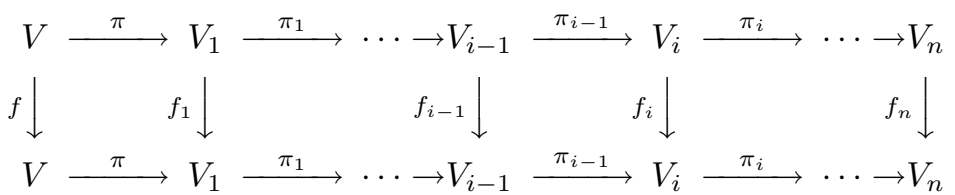

where

(1) $V_{n}$ is a non-simple abelian 3 -fold.

(2) $\pi_{i-1}: V_{i-1} \rightarrow V_{i}$ is (the inverse of) the blow-up along a smooth elliptic curve $C_{i}$ such that $f_{i}^{-1}\left(C_{i}\right)=C_{i}$.

(3) $f_{i}$ is a nontrivial surjective endomorphism of $V_{i}$.

The proposition immediately follows from the following assertions.

Assertions. For all $0 \leq i \leq n$, (here we put $V_{0}:=V$ )

$(1)_{i} V_{i}$ has the structure of an elliptic bundle $p_{i}: V_{i} \rightarrow T_{i}$ over a smooth algebraic surface $T_{i}$ which is birational to an abelian surface.

$(2)_{i} C_{i}$ is contained in a fiber of $p_{i}$.

$(3)_{i} f_{i}$ is compatible with $p_{i}$ and induces an automorphism $g_{i}$ of $T_{i}$.

Proof. We shall prove by a descending induction on $i$. Since $f_{n}^{-1}\left(C_{n}\right)=$ $C_{n}$ and $f_{n}$ is not an isomorphism, the restriction of $f_{n}$ to $C_{n}$ is not a translation in the group law of an elliptic curve $C_{n}$. Hence $f_{n}$ has a fixed point $o$ in $C_{n}$. $V_{n}$ and $C_{n}$ are endowed with the algebraic group structures with $o$ as the identity 
element and $C_{n}$ is an abelian subvariety of $V_{n}$. Let $T_{n}:=V_{n} / C_{n}$ be the quotient of an abelian 3-fold $V_{n}$ by an elliptic curve $C_{n}$. $T_{n}$ is an abelian surface and the natural projection $p_{n}: V_{n} \rightarrow T_{n}$ is an elliptic bundle with typical fiber and structure group $C_{n}$. By the property (2), $f_{n}$ induces an endomorphism $g_{n}$ of $T_{n}$ such that $p_{n} \circ f_{n}=g_{n} \circ p_{n}$. Since $g_{n}$ is a finite étale covering of $T_{n}, g_{n}$ is an automorphism of $T_{n}$ by the property (2). Thus all the assertions hold true for $i=n$.

Next suppose that the assertions hold true for $i+1 . V_{i}$ is the blowing-up of $V_{i+1}$ along $C_{i+1}$ which is the fiber of the elliptic bundle $p_{i+1}: V_{i+1} \rightarrow T_{i+1}$. Hence $V_{i}$ is also an elliptic bundle over $T_{i}$, which is obtained by blowing-up $T_{i+1}$ at $Q_{i+1}:=p_{i+1}\left(C_{i+1}\right)$. By $(3)_{i+1}$ and the rigidity lemma, $f_{i}$ maps each fiber of $p_{i}$ to a fiber of $p_{i}$ and induces a surjective endomorphism $g_{i}$ of $T_{i}$. By Proposition 3.1, $g_{i}$ is an automorphism of $S_{i}$. Since $f_{i}^{-1}\left(C_{i}\right)=C_{i}, C_{i}$ is contained in a fiber of $p_{i}$ by the same argument as in the proof of MAIN THEOREM (A), case (1). Hence all the assertions hold true for $i$.

Proposition 6.2.2. Let $f: V \rightarrow V$ be a nontrivial surjective endomorphism of a smooth projective 3-fold $V$ which is birational to the direct product of a K3 surface and an elliptic curve. Then

(1) $V$ is isomorphic to the direct product $S \times E$ of an elliptic curve $E$ and a nonsingular algebraic surface $S$ which is birational to a $K 3$ surface.

(2) $f$ preserves the elliptic bundle structure $p: V \cong S \times E \rightarrow S$ (resp. the Albanese map $q: V \cong S \times E \rightarrow E$ ) of $V$, which is the first (resp. the second) projection and induces an automorphism $g$ (resp. an endomorphism $h$ ) of $S$ (resp. of $E)$.

Proof. By taking a suitable power of $f$, let $f_{n}: V_{n} \rightarrow V_{n}$ be the minimal reduction of $f: V \rightarrow V$. Then by the proof of Theorem 5.4, $V_{n}$ is isomorphic to the direct product $S_{n} \times E$ of an elliptic curve $E$ and a nonsingular $K 3$ surface $S_{n}$. We have the following commutative diagram:

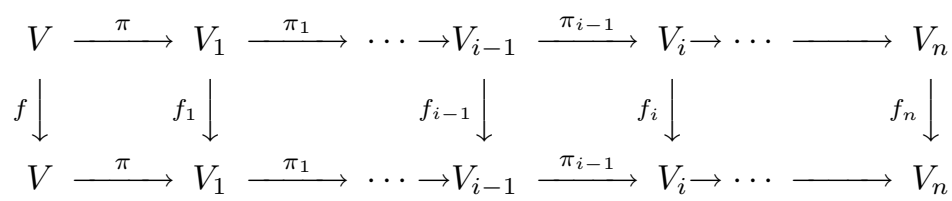

where (1) $\pi_{i-1}: V_{i-1} \rightarrow V_{i}$ is (the inverse of) the blow-up along an elliptic curve $C_{i}$ such that $f_{i}^{-1}\left(C_{i}\right)=C_{i}$,

(2) $f_{i}$ is a nontrivial surjective endomorphism of $V_{i}$.

The proposition immediately follows from the following assertions. 
Assertions. For all $0 \leq i \leq n$, (here we put $V_{0}:=V$ )

$(1)_{i}$ There exists an isomorphism $V_{i} \cong S_{i} \times E$, where $S_{i}$ is a smooth algebraic surface which is birational to a $K 3$ surface.

$(2)_{i} C_{i}$ is contained in fibers of the first projection $p_{i}: V_{i} \rightarrow S_{i}$.

$(3)_{i} f_{i}$ preserves the elliptic bundle structure $p_{i}: V_{i} \rightarrow S_{i}$ (resp. the Albanese map $q_{i}: V_{i} \rightarrow E$ ) of $V_{i}$, which is the first (resp. the second) projection and induces an automorphism $g_{i}$ of $S_{i}$ (resp. an endomorphism $h_{i}$ of $E$ ).

We shall prove this by a descending induction on $i$. For $i=n,(1)_{n}$ is clear by construction and $(3)_{n}$ follows from Corollary 2.13 and the universality of the Albanese map. By $(3)_{n}$ and the property that $f_{n}^{-1}\left(C_{n}\right)=C_{n},(2)_{n}$ follows by the same reason as in the proof of Proposition 6.2.1.

Next suppose that the assertions hold true for $i+1 . V_{i}$ is the blowingup of $V_{i+1}$ along $C_{i+1}$ which is the fiber of the first projection $p_{i+1}: V_{i+1} \cong$ $S_{i+1} \times E \rightarrow S_{i+1}$. Hence we have $V_{i} \cong S_{i} \times E$, where $S_{i}$ is obtained by blowingup $S_{i+1}$ at $Q_{i+1}:=p_{i+1}\left(C_{i+1}\right)$. By Propositions 2.12 and 3.1, $f_{i} \in \operatorname{End}\left(X_{i}\right)$ can be decomposed as $f_{i}=g_{i} \times h_{i}$, where $g_{i} \in \operatorname{Aut}\left(S_{i}\right)$ and $h_{i} \in \operatorname{End}(E)$. Hence this implies $(3)_{i}$. By $(3)_{i}$ and the property that $f_{i}^{-1}\left(C_{i}\right)=C_{i},(2)_{i}$ follows by the same way as in the proof of Proposition 6.2.1.

Having Propositions 6.2.1 and 6.2.2 at our disposal, we are ready to prove MAIN THEOREM (A) in the case (3).

Proof of MAIN THEOREM (A). Case (3): $\kappa(X)=0$.

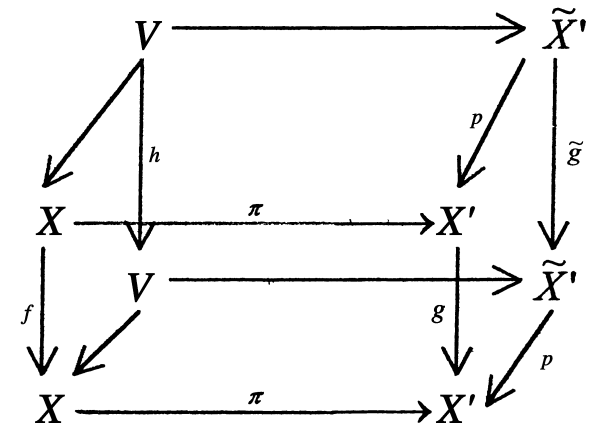

Let $f: X \rightarrow X$ be a nontrivial surjective endomorphism of a smooth projective 3-fold $X$ with $\kappa(X)=0$. By replacing $f$ by a suitable power of $f$, let $g: X^{\prime} \rightarrow X^{\prime}$ be a minimal reduction of $f: X \rightarrow X$ and $\pi: X \rightarrow X^{\prime}$ a succession of extremal contractions of type (E1). We have $g \circ \pi=\pi \circ f$. By Theorem 5.4, a suitable finite Galois étale covering $p: \tilde{X}^{\prime} \rightarrow X^{\prime}$ of $X^{\prime}$ 
is isomorphic either to an abelian 3-fold or the direct product of a smooth $K 3$ surface and an elliptic curve. Moreover, there exists $\tilde{g} \in \operatorname{End}\left(\tilde{X}^{\prime}\right)$ such that $g \circ p=p \circ \tilde{g}$. Then $V:=X \underset{X^{\prime}}{\times} \tilde{X}^{\prime}$ is also nonsingular and birationally equivalent to $\tilde{X}^{\prime}$. And the natural projection $V \rightarrow X$ is a finite Galois étale covering of $X$. The endomorphism $f \times \tilde{g}: X \times \tilde{X}^{\prime} \rightarrow X \times \tilde{X}^{\prime}$ induces a nontrivial surjective endomorphism $h$ of $V$ which satisfies the above commutative diagram. If necessary, we replace $h$ (hence $f$ ) by a suitable power of $h$ (hence $f$ ). Now we shall consider the two cases:

Case $(\alpha) . \quad \tilde{X}^{\prime}$ is isomorphic to an abelian 3-fold.

If $V \rightarrow \tilde{X}^{\prime}$ is an isomorphism, $X$ is the quotient of an abelian 3-fold $V$ by a finite group and is minimal. Next suppose that $V \rightarrow \tilde{X}^{\prime}$ is not an isomorphism. Then by Proposition 6.2.1, $V$ has the structure of an elliptic bundle $q: V \rightarrow \tilde{T}$ over a smooth algebraic surface $\tilde{T}$ which is birational to an abelian surface. It is easy to see that the Galois group $G:=\operatorname{Gal}(V / X)$ preserves the elliptic bundle structure $q: V \rightarrow \tilde{T}$ and induces a finite automorphism group $G^{\prime}$ of $\tilde{T}$. Hence the natural projection $\rho: X \cong V / G \rightarrow \tilde{T} / G^{\prime}=: T$ is a Seifert elliptic fiber space over $T$. Since $f \in \operatorname{End}(X)$ is induced by $h \in \operatorname{End}(V)$ and $X \cong V / G, h$ is compatible with the action of $G$. By Proposition 6.2.1, $h \in \operatorname{End}(V)$ preserves the elliptic bundle $q: V \rightarrow \tilde{T}$ and induces $g^{\prime} \in \operatorname{Aut}(\tilde{T})$. Hence $g^{\prime}$ is also compatible with the action of $G^{\prime} \cong \operatorname{Gal}(\tilde{T} / T)$ and induces $\lambda \in \operatorname{Aut}(T)$.

Case $(\beta) . \quad \tilde{X}^{\prime}$ is isomorphic to the direct product of a smooth $K 3$ surface and an elliptic curve. Then by Proposition 6.2.2, there exists an isomorphism $V \cong \tilde{T} \times E$, where $\tilde{T}$ is birational to a smooth $K 3$ surface and $E$ is an elliptic curve. Moreover, $h \in \operatorname{End}(V)$ can be decomposed as $h=h_{1} \times h_{2}$, where $h_{1} \in \operatorname{Aut}(\tilde{T})$ and $h_{2} \in \operatorname{End}(E)$. By Proposition 2.12, the Galois group $\tilde{G}:=$ $\operatorname{Gal}(V / X)$ preserves the first projection $p_{1}: V \rightarrow \tilde{T}$ (resp. the second projection $p_{2}: V \rightarrow E$ ) and induces a finite automorphism group $G$ (resp. $G^{\prime}$ ) of $\tilde{T}$ (resp. E). The natural projection $\rho_{1}: X:=V / \tilde{G} \rightarrow \tilde{T} / G=: T$ is a Seifert elliptic fiber space over $T$. Moreover, the natural projection $\rho_{2}: X:=V / \tilde{G} \rightarrow$ $E / G^{\prime}=: W$ is a Seifert fiber space over the curve $W$, whose general fiber is birational to a $K 3$ surface or an Enriques surface. By the same reason as in the proof of Case $(\alpha), f \in \operatorname{End}(X)$ preserves the above fiber space structures and induces an automorphism $\alpha$ of $T$ (resp. an endomorphism $\beta$ of $W$ ).

Example 6.2. Under the same situation as in Example 5.2 in Section 5 , let $u: T^{\prime} \rightarrow T$ be the blowing-up at one point $t \in T$. Then $Y^{\prime}:=\underset{T}{\times} T^{\prime}$ is nonsingular and the natural projection $h^{\prime}: Y^{\prime} \rightarrow T^{\prime}$ is an elliptic fiber bundle 
over $T^{\prime}$. Clearly $\mu: Y \rightarrow Y$ can be naturally extended to give a nontrivial surjective endomorphism $\mu^{\prime}$ of $Y^{\prime}$. The composite map $\varphi: Y^{\prime} \rightarrow Y \stackrel{g}{\longrightarrow}$ $E /\langle\tau\rangle \cong \mathbf{P}^{1}$ induces on $Y^{\prime}$ a Seifert fiber space structure whose general fiber is birational to a $K 3$ surface. $\mu^{\prime}$ induces a nontrivial surjective endomorphism $w$ of $\mathbf{P}^{1}$ such that $\varphi \circ \mu^{\prime}=w \circ \varphi$, but is an identity map when restricted to each fiber of $\varphi$.

6.3. Finally we shall consider the case $(2)$ where $\kappa(X)=1$.

Proof of MAIN THEOREM(A). Case $(2): \kappa(X)=1$.

Let $f: X \rightarrow X$ be a nontrivial surjective endomorphism of a smooth projective 3 -fold $X$ with $\kappa(X)=1$. By taking a suitable power of $f$, we have the following commutative diagram:

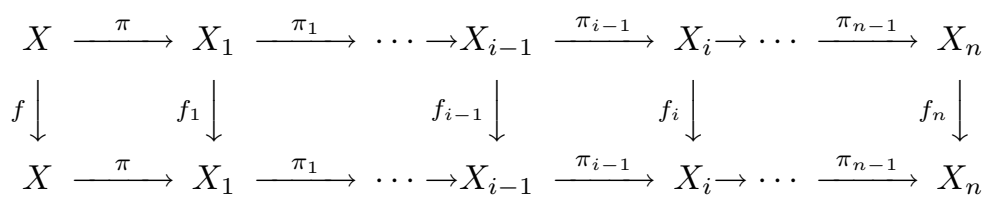

where

(1) $f_{i}$ is a nontrivial surjective endomorphism of $X_{i}$,

(2) $\pi_{i-1}: X_{i-1} \rightarrow X_{i}$ is (the inverse of) the blow-up along a smooth elliptic curve $C_{i}$ on $X_{i}$ such that $f_{i}^{-1}\left(C_{i}\right)=C_{i}$,

(3) $X_{n}$ is the unique nonsingular minimal model of $X$ (cf. Theorem 5.10) and $f_{n}$ is then a minimal reduction of $f$.

Let $\varphi: X_{n} \rightarrow C$ be the Iitaka fibration of $X_{n}$. By Proposition 5.7, the 'general' fiber of $\varphi$ is either an abelian surface or a hyperelliptic surface.

From now on, we assume that the general fiber of $\varphi$ is a hyperelliptic surface.

Step 1.

Lemma 6.3.1. If we put $\phi_{i}:=\varphi \circ \pi_{n-1} \circ \cdots \circ \pi_{i}: X_{i} \rightarrow C$ and $\phi_{n}:=$ $\varphi, C_{i}$ is contained in a fiber of $\phi_{i}$ for all $i \geq 0$.

Proof. Assume that $\phi_{i}\left(C_{i}\right)=C$. Since $f_{i} \in \operatorname{End}\left(X_{i}\right)$ is a finite étale covering, $f_{i}$ induces $v \in \operatorname{Aut}(C)$ such that $\phi_{i} \circ f_{i}=v \circ \phi_{i}$ by Proposition 2.5. Hence for a general fiber $F$ of $\phi_{i}, f_{i}^{*} F$ is also a general fiber of $\phi_{i}$ and numerically equivalent to $F$. Since $f_{i}^{-1}\left(C_{i}\right)=C_{i}$, we have $\left(F, C_{i}\right)=\left(f_{i}^{*} F, C_{i}\right)=$ 
$\left(F,\left(f_{i}\right)_{*} C_{i}\right)=\operatorname{deg}\left(f_{i}\right) \times\left(F, C_{i}\right)$. Since $\left(F, C_{i}\right)>0$, we have $\operatorname{deg}\left(f_{i}\right)=1$ and $f_{i}$ is an isomorphism. This is a contradiction.

By Theorem 5.10, we have the following diagram:

$$
X_{n} \stackrel{g_{n}}{\longrightarrow} T_{n} \stackrel{h_{n}}{\longrightarrow} C
$$

where

(1) $g_{n}: X_{n} \rightarrow T_{n}$ is a Seifert elliptic fiber space over a normal surface $T_{n}$ with at most quotient singularities, $h_{n}: T_{n} \rightarrow C$ is a $\mathbf{P}^{1}$-fiber space, and $\varphi=h_{n} \circ g_{n}$.

(2) $f_{n} \in \operatorname{End}\left(X_{n}\right)$ induces $p_{n} \in \operatorname{End}\left(T_{n}\right)$ and $q_{n} \in \operatorname{Aut}(C)$ which are compatible with the above diagram.

By Lemma 6.3.1, the following two Cases can occur:

Case (A). $C_{n}$ is contained in a fiber of $g_{n}$.

Case (B). $D_{n}:=g_{n}\left(C_{n}\right)$ is a rational curve contained in a fiber of $h_{n}$.

Step 2. First, we shall consider the case (A).

Lemma 6.3.2. In this case, $p_{n}$ is an automorphism of $T_{n}$.

Proof. By Theorem 5.10, there exists a finite étale covering $\gamma: \tilde{X}_{n} \rightarrow$ $X_{n}$ of $X_{n}$ such that $\tilde{X}_{n} \cong \tilde{T}_{n} \times E$, where $E$ is an elliptic curve and $\tilde{T}_{n}$ is a nonsingular minimal algebraic surface with $\kappa=1$. Then $S:=\gamma\left(\tilde{T}_{n} \times\{0\}\right)$ is a nonsingular minimal algebraic surface with $\kappa(S)=1$ and mapped surjectively onto $T_{n}$ by $g_{n}$. Let $S^{\prime}$ be the strict transform of $S$ by $\pi_{n-1}: X_{n-1} \rightarrow X_{n}$. Then $f_{n-1} \in \operatorname{End}\left(X_{n-1}\right)$ defines an infinite descending sequence of finite étale covering between smooth surfaces with $\kappa=1: S^{\prime} \stackrel{f_{n-1}}{\longrightarrow} S^{\prime}{ }_{1} \stackrel{f_{n-1}}{\longrightarrow} S^{\prime}{ }_{2} \stackrel{f_{n-1}}{\longrightarrow}$ $\cdots \rightarrow S^{\prime}{ }_{k} \stackrel{f_{n-1}}{\longrightarrow} S^{\prime}{ }_{k+1} \rightarrow \cdots$, where $S^{\prime}{ }_{0}:=S^{\prime}$ and $S^{\prime}{ }_{k}:=f_{n-1}^{k}\left(S^{\prime}\right)$. Since $S^{\prime}$ is not minimal, Proposition 7.1 implies that $\left.f_{n-1}\right|_{S^{\prime}{ }_{k}}: S^{\prime}{ }_{k} \rightarrow S^{\prime}{ }_{k+1}$ is an isomorphism for a sufficiently large positive integer $k$. Assume that $\operatorname{deg}\left(p_{n}\right)>1$ and we shall derive a contradiction. Let $F$ be the general fiber of $g_{n-1}^{\prime}:=$ $g_{n} \circ \pi_{n-1}: X_{n-1} \rightarrow T_{n}$. Then for large $k$, we have

$$
\left(S^{\prime}{ }_{k+1}, F\right)=\left(\left(f_{n-1}\right)_{*} S^{\prime}{ }_{k}, F\right)=\left(S^{\prime}{ }_{k}, f_{n-1}^{*} F\right)=\operatorname{deg}\left(p_{n}\right) \times\left(S^{\prime}{ }_{k}, F\right) .
$$


Hence we have $(\mathbf{P}) \operatorname{deg}\left(g_{n-1}^{\prime}: S^{\prime}{ }_{k} \rightarrow T_{n}\right) \rightarrow \infty$ as $k \rightarrow \infty$. We have the following commutative diagram:

$$
\begin{aligned}
& S_{k}^{\prime} \stackrel{f_{n-1}}{\longrightarrow} S_{k+1}^{\prime} \\
& g^{\prime}{ }_{n-1} \mid S^{\prime}{ }_{k} \downarrow \downarrow \quad\left\lfloor g^{\prime}{ }_{n-1} \mid S^{\prime}{ }_{k+1}\right. \\
& T_{n} \stackrel{p_{n}}{\longrightarrow} T_{n}
\end{aligned}
$$

Now, if we take a sufficiently ample smooth curve $D$ on $T_{n}$ with $g(D) \geq 2$, $W:=\left(g_{n-1}^{\prime}\right)^{-1}(D)$ is a nonsingular minimal Seifert elliptic surface over $D$. $f_{n} \in \operatorname{End}\left(X_{n-1}\right)$ and $p_{n} \in \operatorname{End}\left(T_{n-1}\right)$ define an infinite sequence of finite étale covering between minimal smooth surfaces with $\kappa=1$,

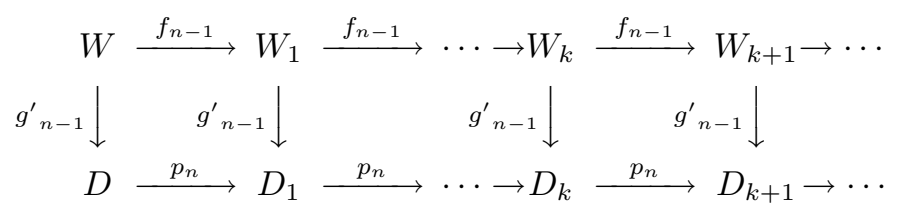

where $W_{0}:=W, W_{k}:=f_{n-1}^{k}(W), D_{0}:=D$ and $D_{k}:=p_{n}^{k}(D)$. By construction, $\Delta_{k}:=S^{\prime}{ }_{k} \cap W_{k}$ is also irreducible. Hence we have $f_{n-1}\left(\Delta_{k}\right)=\Delta_{k+1}$ and $\left.f_{n-1}\right|_{\Delta_{k}}: \Delta_{k} \rightarrow \Delta_{k+1}$ is an isomorphism for $k \gg 0$. Hence, if we restrict the isomorphism $\left.f_{n-1}\right|_{S^{\prime}{ }_{k}}: S^{\prime}{ }_{k} \cong S^{\prime}{ }_{k+1}$ to the finite étale covering $\left.f_{n-1}\right|_{W_{k}}$ : $W_{k} \rightarrow W_{k+1}$ between $g_{n-1}^{\prime}$-vertical divisors of $X_{n-1}$, we have $\operatorname{deg}\left(g_{n-1}^{\prime}\right.$ : $\left.\Delta_{k} \rightarrow D_{k}\right)=\operatorname{deg}\left(g_{n-1}^{\prime}: \Delta_{k+1} \rightarrow D_{k+1}\right)$, since $p_{n}: D_{k} \rightarrow D_{k+1}$ is an isomorphism for sufficiently large $k$ by Proposition 7.1. This contradicts and the proof is finished.

MAIN THEOREM in the subcase (A) is an immediate consequence of the following Assertions.

Assertions. For all $0 \leq i \leq n$, (here we put $X_{0}:=X$ ).

$(1)_{i}$ We have the following diagram of fiber spaces: $X_{i} \stackrel{g_{i}}{\longrightarrow} T_{i} \stackrel{h_{i}}{\longrightarrow} C$, where $g_{i}: X_{i} \rightarrow T_{i}$ is a Seifert elliptic fiber space over a normal surface $T_{i}$ with at most quotient singularities, $h_{i}: T_{i} \rightarrow C$ is a $\mathbf{P}^{1}$-fiber space, and the 'general' fiber of $\phi_{i}:=h_{i} \circ g_{i}$ is a hyperelliptic surface.

$(2)_{i} f_{i}$ induces $p_{i} \in \operatorname{Aut}\left(T_{i}\right)$ and $\beta \in \operatorname{Aut}(C)$ which is compatible with the above diagram.

$(3)_{i}$ A suitable finite étale covering $\tilde{X}_{i}$ of $X_{i}$ is isomorphic to the direct product $\tilde{T}_{i} \times E$ of an elliptic curve $E$ and a nonsingular algebraic surface $\tilde{T}_{i}$ with $\kappa=1$.

$(4)_{i} C_{i}$ is contained in fibers of $g_{i}$. 
Proof. We shall prove this by a descending induction on $i$. For $i=n$, the assertions hold true by assumption and Lemma 6.3.2. Next suppose that the assertions hold true for $i+1$. Then $(1)_{i}$ and $(3)_{i}$ follow from Proposition 6.1.1 and there exists a birational morphism $\tau_{i}: T_{i} \rightarrow T_{i+1}$ such that $\tau_{i} \circ g_{i}=g_{i+1} \circ \pi_{i}$. Put $h_{i}:=h_{i+1} \circ \tau_{i}$. Since $g_{i}: X_{i} \rightarrow T_{i}$ is equidimensional, $f_{i} \in \operatorname{End}\left(X_{i}\right)$ induces $p_{i} \in \operatorname{End}\left(T_{i}\right)$ such that $p_{i} \circ g_{i}=g_{i} \circ f_{i}$ and $\tau_{i} \circ p_{i}=p_{i+1} \circ \tau_{i}$ by the rigidity lemma. Since $p_{i}$ is a finite birational morphism, it is an automorphism of $T_{i}$ by Zariski's main theorem. Thus $(2)_{i}$ is derived. Since $f_{i}^{-1}\left(C_{i}\right)=C_{i}$ and $p_{i} \in \operatorname{Aut}\left(T_{i}\right),(4)_{i}$ follows by the same reason as in the proof of Case (1).

Step 3. Next, we shall consider the case (B).

Then $S_{n}:=g_{n}^{-1}\left(D_{n}\right)$ is a Seifert elliptic surface over $D_{n}$ which contains $C_{n}$ and may be singular. The normalization $n: U_{n} \rightarrow S_{n}$ of $S_{n}$ is nonsingular and a suitable finite étale covering $\tilde{U}_{n}$ of $U_{n}$ is isomorphic to the direct product of a smooth curve and an elliptic curve $E$. Since $\varphi_{n}: X_{n} \rightarrow C$ is projective and the general fiber of $\varphi_{n}$ is a hyperelliptic surface, we have $\kappa\left(U_{n}\right) \leq 0$ by the lower semi-continuity of the Kodaira dimension under degenerations of surfaces (cf. [U2], [N5]). Hence the following two Cases can occur:

Case (a). We have $\kappa\left(U_{n}\right)=-\infty$ and $U_{n}$ is an elliptic ruled surface.

Case (b). We have $\kappa\left(U_{n}\right)=0$ and $U_{n}$ is a hyperelliptic surface. In fact, since $\chi\left(\mathscr{O}_{U_{n}}\right)=0, U_{n}$ is neither a $K 3$ surface nor an Enriques surface. Since $U_{n}$ is a minimal Seifert elliptic surface over $\mathbf{P}^{1}$ and $\kappa\left(U_{n}\right)=0$, it must have multiple fibers. Hence $U_{n}$ is not an abelian surface. Hence by the classification theory of algebraic surfaces, it is a hyperelliptic surface.

Lemma 6.3.3. Case (a) cannot occur.

Proof.

Claim. We have $f_{n}^{-1}\left(S_{n}\right)=S_{n}$ set-theoretically.

Proof. Since $C_{n}=f_{n}^{-1}\left(C_{n}\right) \subset f_{n}^{-1}\left(S_{n}\right)=g_{n}^{-1}\left(p_{n}^{-1}\left(D_{n}\right)\right)$, we have $D_{n}:=$ $g_{n}\left(C_{n}\right) \subset p_{n}^{-1}\left(D_{n}\right)$. Assume that on $T_{n}$ there exists an irreducible curve $B(\neq$ $\left.D_{n}\right)$ contained in $p_{n}^{-1}\left(D_{n}\right)$. Then the elliptic surface $g_{n}^{-1}(B)$ over $B$ is mapped surjectively onto $S_{n}$ by $f_{n}$. Hence there exists an irreducible curve $H$ on $g_{n}^{-1}(B)$ such that $f_{n}(H)=C_{n}$. Since $f_{n}\left(g_{n}^{-1}(B) \cap S_{n}\right)=f_{n}\left(g_{n}^{-1}\left(B \cap D_{n}\right)\right)$ is contained in fibers of $g_{n}$ and $C_{n}=f_{n}(H)$ is not contained in fibers of $g_{n}$, we have $H \not \subset S_{n}$ and hence $H \neq C_{n}$. This contradicts the assumption that $f_{n}^{-1}\left(C_{n}\right)=C_{n}$. Hence we have $D_{n}=p_{n}^{-1}\left(D_{n}\right)$ and $f_{n}^{-1}\left(S_{n}\right)=g_{n}^{-1}\left(D_{n}\right)=S_{n}$. 
$f_{n}$ can be lifted to an étale endomorphism $\bar{f}_{n}$ of $U_{n}$. By Lemma 5.11, $\bar{f}_{n}$ preserves the Seifert elliptic surface structure of $U_{n}$ and induces an automorphism of the base curve $\hat{D}_{n} \cong \mathbf{P}^{1}$. Let $\bar{C}_{n}$ be the strict transform of $C_{n}$ by $n$. By the fact that $\bar{f}_{n}^{-1}\left(\bar{C}_{n}\right)=\bar{C}_{n}$ and the above claim, we have $\operatorname{deg}\left(f_{n}\right)=\operatorname{deg}\left(\left.f_{n}\right|_{S_{n}}\right)=\operatorname{deg}\left(\bar{f}_{n}\right)=1$ and $f_{n}$ is an isomorphism. This is a contradiction.

Now we shall consider the case (b).

Lemma 6.3.4. $\quad$ There exists another commutative diagram of fiber spaces:

$$
X_{n} \stackrel{\alpha_{n}}{\longrightarrow} A_{n} \stackrel{\beta n}{\longrightarrow} C
$$

such that

(1) $\alpha_{n}: X_{n} \rightarrow A_{n}$ is the relative Albanese map of $X_{n}$ over $C$ (cf. [F3]) and gives $X_{n}$ an equidimensional elliptic fiber space structure over a normal surface $A_{n}$ with at most quotient singularities.

(2) $\beta_{n}: A_{n} \rightarrow C$ is an elliptic fibration over the curve $C$.

(3) $\varphi=\beta_{n} \circ \alpha_{n}: X_{n} \rightarrow C$ is the Iitaka fibration of $X_{n}$.

(4) $f_{n} \in \operatorname{End}\left(X_{n}\right)$ induces an endomorphism (resp. an automorphism) of $A_{n}$ (resp. C) and preserves the above fiber space structures.

Proof. We shall employ almost all the same method as in the proof of Theorem 5.10. The only minor change is that, instead of taking the relative generic quotient of $X_{n}$ by the relative automorphism group of $X_{n}$ over $C$, we consider the relative Albanese map of $X_{n}$ over $C$. After performing a finite succession of flpos to $X_{n}$, we get another minimal model $X^{\prime}{ }_{n}$ which satisfies the above requirements $(1) \sim(3)$. However, since $X_{n}$ is the unique minimal model of $X$ by Theorem 5.10, we have an isomorphism $X_{n}^{\prime} \cong X_{n}$. (4) is derived from Propositions 2.5 and 5.8.

Proof of Case (B) continued. Thus $X_{n}$ has 2 different elliptic fibrations.

Lemma 6.3.5. $\quad C_{n}$ is contained in a fiber of $\alpha_{n}$.

Proof. By the composite map $U_{n} \stackrel{n}{\longrightarrow} S_{n} \subset X_{n} \stackrel{\varphi}{\longrightarrow} C, \varphi \circ n\left(U_{n}\right)$ is a point on $C$. Since $\alpha_{n}$ is equidimensional, $\alpha_{n}\left(S_{n}\right)=\alpha_{n} \circ n\left(U_{n}\right)(=: \gamma)$ is an irreducible curve contained in a fiber of $\beta_{n}$. Hence by the rigidity lemma, the hyperelliptic surface $U_{n}$ has two nonisomorphic elliptic fibrations $g_{n} \circ n: U_{n} \rightarrow$ $g_{n} \circ n\left(U_{n}\right)(:=\Delta)$ and $\alpha_{n} \circ n: U_{n} \rightarrow \gamma$. Since $\bar{C}_{n}$ is a smooth elliptic curve on 
$U_{n}$, we have $\left(\bar{C}_{n}\right)_{U_{n}}^{2}=0$. Note that $\bar{C}_{n}$ is mapped surjectively onto $\Delta$ by $g_{n} \circ n$. Hence by the Fact after the proof of (5.7), $\bar{C}_{n}$ is in the fiber of $\alpha_{n} \circ n$.

If we take a sufficiently ample smooth curve $\Gamma$ on $T_{n}, g_{n}^{-1}(\Gamma)$ is a smooth surface with $\kappa=1$ and mapped surjectively onto $A_{n}$ by $\alpha_{n}$. By the assumption that $f_{n}^{-1}\left(C_{n}\right)=C_{n}$ and Lemma 6.3.5, $f_{n}$ induces an automorphism of $A_{n}$ and is compatible with the above diagram for the same reason as in the proof of Lemma 6.3.2. Hence $\alpha_{n}: X_{n} \rightarrow A_{n}$ is a Seifert elliptic fiber space by the same argument as in the proof of Theorem 5.1. Thus MAIN THEOREM for the case (B) immediately follows from the following Assertions:

Assertions. For all $0 \leq i \leq n,\left(\right.$ here we put $X_{0}:=X$ )

(1) ${ }_{i}$ There exists a commutative diagram of fiber spaces: $X_{i} \stackrel{\alpha_{i}}{\longrightarrow} A_{i} \stackrel{\beta_{i}}{\longrightarrow}$ $C$, where $\alpha_{i}: X_{i} \rightarrow A_{i}$ is the relative Albanese map of $X_{i}$ over $C$ and gives the Seifert elliptic fiber space structure over a normal surface $A_{i}$ with at most quotient singularities, and $\beta_{i}: A_{i} \rightarrow C$ is an elliptic surface and the general fiber of $\phi_{i}:=\beta_{i} \circ \alpha_{i}$ is a hyperelliptic surface.

$(2)_{i} f_{i}$ induces automorphisms of $A_{i}$ and $\mathrm{C}$ which are compatible with the above diagram.

$(3)_{i}$ A suitable finite étale covering $\tilde{X}_{i}$ of $X_{i}$ is isomorphic to the direct product $\tilde{A}_{i} \times E$ of an elliptic curve $E$ and a smooth surface $\tilde{A}_{i}$ with $\kappa=1$.

$(4)_{i} C_{i}$ is contained in fibers of $\alpha_{i}$.

Proof. We shall prove by a descending induction on $i$. For $i=n,(3)_{n}$ follows from Theorem [N] in Section 5, and the other claims follow from the above remark. The rest of the proof is completely the same as in Step 2 in Case (A), so we omit it.

We conclude with a remark concerning the last theorem.

Remark 6.3. (1) In Case (B), each fiber of $\beta_{n}: A_{n} \rightarrow C$ is a nonsingular elliptic curve. Moreover, a suitable finite étale covering $W$ of $X_{n}$ is isomorphic to the direct oroduct $\tilde{C} \times E \times E^{\prime}$ of a smooth curve $\tilde{C}$ with $g(\tilde{C}) \geq 2$ and elliptic curves $E$ and $E^{\prime}$. In fact, suppose that an irreducible rational curve $e$ is contained in a fiber of $\beta_{n}$. Let $V$ be the normalization of $\alpha_{n}^{-1}(e)$. By the same reason as above, $V$ is either a smooth relatively minimal elliptic ruled surface or a hyperelliptic surface. Suppose that $V$ is an elliptic ruled surface. Since $\varphi_{n}(V)$ is a point on $C, g_{n}$ is equidimensional and $T_{n}$ is a $\mathbf{P}^{1}$-fiber space over $C$, $g_{n}(V)$ is a rational curve contained in a fiber of $h_{n}$. Hence $V$ has two different elliptic fibrations over $\mathbf{P}^{1}$ by the rigidity lemma. This is a contradiction. Next, 
suppose that $V$ is a hyperelliptic surface. By the same reason as above, $V$ has two different elliptic fibrations over $\mathbf{P}^{1}$. This is a contradiction. Hence each fiber of $\beta_{n}$ is an elliptic curve. Let $\tilde{A}_{n} \rightarrow C^{\prime} \rightarrow C$ be the Stein factorization of the composite map $\tilde{A}_{n} \rightarrow A_{n} \stackrel{\beta_{n}}{\longrightarrow} C$. Then $\tilde{A}_{n}$ is a nonsingular Seifert elliptic surface over $C^{\prime}$ and a suitable finite étale covering $D$ of $\tilde{A}_{n}$ is isomorphic to the direct product of a smooth curve $\tilde{C}$ with $g(\tilde{C})>1$ and an elliptic curve $E^{\prime}$. Since $\tilde{X}_{n} \cong \tilde{A}_{n} \times E$, the claim follows.

(2) As the proof of MAIN THEOREM shows, the unique nonsingular minimal model $X_{n}$ of $X$ has exactly two different elliptic fibrations: One is a Seifert elliptic fiber space $g_{n}: X_{n} \rightarrow T_{n}$ given by Fujiki quotient and the other is given by a relative Albanese map $\alpha_{n}: X_{n} \rightarrow A_{n}$. In most cases, $X$ is obtained from $X_{n}$ by blowing-up successively along fibers of $g_{n}$. And this approach eventually turned out to be the most effective. If we blow-up $X_{n}$ along a fiber of $\alpha_{n}$, we sometimes encounter serious troubles as the next example shows.

Example 6.3. $\quad$ Under the same situation as in Example 5.3 in Section 5 , take a smooth point $t$ on $T$ such that $g^{-1}(t)$ is a regular fiber. Let $X^{\prime}$ be the blowing-up of $X$ along $g^{-1}(t)$. Clearly $\varphi$ can be naturally extended to give a nontrivial surjective endomorphism $\varphi^{\prime}$ of $X^{\prime}$. On the other hand, let $v: Y \rightarrow X$ be the blow-up of $X$ along a regular fiber $F$ of $h$. Then $Y$ has no nontrivial surjective endomorphism.

This immediately follows from Remark 6.3. But here we give another simpler proof. Assume the contrary and let $u: Y \rightarrow Y$ be a nontrivial surjective endomorphism. By Proposition 4.4 and Theorem 4.8, a suitable power $v:=$ $u^{k}(k>0)$ of $u$ induces a nontrivial surjective endomorphism $w: X \rightarrow X$ such that $\pi \circ v=w \circ \pi$ and $w^{-1}(F)=F$. Hence by the rigidity lemma, there exists a surjective endomorphism $w^{\prime}$ of $C \times E^{\prime}$ such that $h \circ w=w^{\prime} \circ h$. Since $w: X \rightarrow X$ induces an automorphism of $C$ by Proposition 2.5, $w^{\prime}$ is an étale endomorphism. Since $w^{-1}(F)=F, w^{\prime}$ is an automorphism of $C \times E^{\prime}$. Hence by the same argument as in the proof of Case (1), $h: X \rightarrow C \times E^{\prime}$ is a Seifert elliptic fibration. In particular, there exist no rational curves contained in fibers of $h$. This contradicts the assumption that $\chi\left(\mathscr{O}_{S}\right) \neq 0$.

\section{$\S 7 . \quad$ Applications}

In this section, we shall study the structure of nonsingular surfaces with $\kappa \geq 0$ and smooth projective 3 -folds with $\kappa=2$ which admit an infinite descending sequence of nonisomorphic finite étale coverings. 
Proposition 7.1. Let $S_{1} \stackrel{f_{1}}{\longrightarrow} S_{2} \stackrel{f_{2}}{\longrightarrow} \cdots \rightarrow S_{n} \stackrel{f_{n}}{\longrightarrow} S_{n+1} \stackrel{f_{n+1}}{\longrightarrow}$ .. be an infinite sequence of nonisomorphic finite étale coverings between smooth algebraic surfaces $S_{n}$ 's with $\kappa\left(S_{n}\right) \geq 0$. Then for all $n, S_{n}$ is minimal and one of the following cases occurs:

Case (1). If $\kappa\left(S_{n}\right)=1$ for all $n$, the Iitaka fibration $\varphi_{n}: S_{n} \rightarrow C_{n}$ gives the unique structure of a minimal Seifert elliptic fibration. Moreover,

1. A suitable finite étale covering $T_{n}$ of $S_{n}$ is isomorphic to the direct product $D_{n} \times E_{n}$ of a smooth curve $D_{n}$ with $g\left(D_{n}\right) \geq 2$ and an elliptic curve $E_{n}$ which is mutually isogeneous to each other.

2. For all $n$, there exists a finite covering $g_{n}: C_{n} \rightarrow C_{n+1}$ such that $\varphi_{n+1} \circ f_{n}=g_{n} \circ \varphi_{n}$. Moreover, $g_{n}$ is an isomorphism for a sufficiently large positive integer $n$.

Case (2). If $\kappa\left(S_{n}\right)=0$ for all $n$, each $S_{n}$ is isomorphic to an abelian surface or a hyperelliptic surface. And only one of the following three cases occurs.

(A) All $S_{n}$ 's are abelian surfaces.

(B) All $S_{n}$ 's are hyperelliptic surfaces.

(C) There exists a positive integer $k$ such that $S_{n}$ is isomorphic to an abelian surface (resp. a hyperelliptic surface) for all $n \leq k$ (resp. for all $n>k)$.

Proof. Step 1. First we show that $S_{n}$ is minimal for all $n$.

Since $\rho\left(S_{n}\right)$ decreases as $n$ increases, there exists a positive integer $p$ such that $\rho\left(S_{n}\right)$ is constant for all $n \geq p$. Assume that $S_{p}$ is not minimal. By applying Proposition 4.2 and its Remark to the nonisomorphic finite étale covering $h:=f_{p}: S_{p} \rightarrow S_{p+1}$, we see that $h^{*}$ and $h_{*}$ give a one-to-one correspondence between the set of $(-1)$-curves on $S_{p}$ and the set of $(-1)$-curves on $S_{p+1}$. On the other hand, since $h$ is finite étale covering and the $(-1)$-curve $e$ on $S_{p+1}$ is simply connected, $h^{-1}(e)$ consists of $d:=\operatorname{deg}(h)(\geq 2)$ disjoint $(-1)$-curves. This is a contradiction. Hence $S_{p}$ is minimal. Since each $f_{n}$ is étale, $\kappa\left(S_{n}\right)$ is constant and $S_{n}$ is minimal. Hence $K_{S_{n}}$ is semi-ample for all $n$.

Step 2. Kollár's theorem [Kol2, Proposition 9.4] implies that $S_{n}$ is not of general type by the same way as in the proof of Corollary 4.11. There remain two Cases to be considered:

Case (1). Suppose that $\kappa\left(S_{n}\right)=1$ for all $n$. The Iitaka fibration $\varphi_{n}$ : $S_{n} \rightarrow C_{n}$ gives the unique structure of an elliptic surface. Since each $f_{i}$ is finite étale, we have $\chi\left(\mathscr{O}_{S_{1}}\right)=\chi\left(\mathscr{O}_{S_{n}}\right) \times \prod_{i=1}^{n-1} \operatorname{deg}\left(f_{i}\right)$ for all $n$. Assume that 
$\chi\left(\mathscr{O}_{S_{1}}\right) \neq 0$. Because $\operatorname{deg}\left(f_{i}\right)>1$ for all $i$, we have $\left|\chi\left(\mathscr{O}_{S_{1}}\right)\right|=\infty$ as $n \rightarrow \infty$ and this is a contradiction. Hence we have $\chi\left(\mathscr{O}_{S_{1}}\right)=0$ and so $\chi\left(\mathscr{O}_{S_{n}}\right)=0$ for all $n$. The first assertion follows from the theory of elliptic surfaces due to Kodaira. Let $F_{n}$ be the general fiber of $\varphi_{n}$. Then we have $\left(K_{S_{n+1}}, f_{n *} F_{n}\right)=$ $\left(f_{n}^{*} K_{S_{n+1}}, F_{n}\right)=\left(K_{S_{n}}, F_{n}\right)=0$. Since $K_{S_{n}} \sim_{Q} \varphi_{n}^{*} L_{n}$ for an ample Q-divisor $L_{n}$ on $C_{n}, \operatorname{Supp}\left(f_{n}\left(F_{n}\right)\right)$ is contained in a fiber of $\varphi_{n+1}$ and the second assertion follows from the rigidity lemma. Since $L_{n} \sim_{Q} g_{n}^{*} L_{n+1}$ for all $n$, we have $\operatorname{deg}\left(L_{1}\right)=\operatorname{deg}\left(L_{n+1}\right) \times \prod_{i=1}^{n} \operatorname{deg}\left(g_{i}\right)$. Suppose that $g_{n}$ is not an isomorphism for infinitely many positive integers $n$. Then, if we let $n \rightarrow \infty$, we have $\operatorname{deg}\left(L_{1}\right)=$ $\infty$ and this is a contradiction. Hence $g_{n}$ is an isomorphism for sufficiently large positive integers $n$.

Case (2). Next suppose that $\kappa\left(S_{n}\right)=0$ for all $n$. We have $\chi\left(\mathscr{O}_{n}\right)=0$ by the same reason as in Case (1). Since $S_{n}$ is minimal, the classification theory of algebraic surfaces implies that $S_{n}$ is either an abelian surface or a hyperelliptic surface. The irregularity $q\left(S_{n}\right)$ decreases as $n$ increases, and we have $q\left(S_{n}\right)=2$ (resp. 1) if and only if $S_{n}$ is an Abelian surface (resp. a hyperelliptic surface). Hence there exists an unique integer $k(0 \leq k \leq \infty)$ such that $S_{n}$ is an abelian surface (resp. a hyperelliptic surface) if $n \leq k$ (resp. $n>k$ ).

The similar method as in the proof of MAIN THEOREM (A), Case (1) yields the following.

Theorem 7.2. Let $X_{1} \stackrel{f_{1}}{\longrightarrow} X_{2} \stackrel{f_{2}}{\longrightarrow} \cdots \rightarrow X_{n} \stackrel{f_{n}}{\longrightarrow} X_{n+1} \stackrel{f_{n+1}}{\longrightarrow}$ .. be an infinite descending sequence of nonisomorphic finite étale coverings between nonsingular projective 3 -folds $X_{n}$ 's with $\kappa\left(X_{n}\right)=2$. Then for all positive integer $n$,

(1) $X_{n}$ has the unique nonsingular minimal model.

(2) $X_{n}$ has the structure of a Seifert elliptic fiber space $\Phi_{n}: X_{n} \rightarrow W_{n}$ over a normal surface $W_{n}$ with at most quotient singularities.

(3) For all $n$, there exists a finite covering $g_{n}: W_{n} \rightarrow W_{n+1}$ such that $\Phi_{n+1} \circ f_{n}=g_{n} \circ \Phi_{n}$. Moreover, $g_{n}$ is an isomorphism for a sufficiently large positive integer $n$.

(4) A suitable finite étale covering $\tilde{X}_{n}$ of $X_{n}$ is isomorphic to the direct product $T_{n} \times E_{n}$ of a smooth algebraic surface $T_{n}$ of general type (not necessarily minimal) and a smooth elliptic curve $E_{n}$ which is mutually isogeneous to each other.

Corollary 7.3. Let $f: X \rightarrow T$ be an elliptic fibration from a smooth projective 4-fold $X$ with $\kappa(X)=3$ onto a normal projective 3 -fold T. Assume 
that $X$ admits a nontrivial surjective endomorphism $\varphi$. Take a sufficiently general hyperplane section $H$ of $T$ such that $Z:=f^{-1}(H)$ is a nonsingular projective 3 -fold with $\kappa(Z)=2$. Then

(1) $Z$ admits a Seifert elliptic fiber space structure $g: Z \rightarrow W$ over a normal surface $W$ with at most quotient singularities,

(2) A suitable finite étale covering $\tilde{Z}$ of $Z$ is isomorphic to the direct product $\tilde{W} \times E$ of an elliptic curve $E$ and a nonsingular algebraic surface $\tilde{W}$ of general type.

\section{Acknowledgements}

The author wishes to express sincere thanks to Professors Shigefumi Mori and Noboru Nakayama for many suggestions. In this paper, the construction of the minimal reduction of a nontrivial endomorphism is slightly different from the author's original method in $[\mathrm{SF}]$. The main improvement, originally suggested by Professor Mori, is that we first take a suitable power $f^{k}(k>0)$ of a given endomorphism $f$. This considerably simplifies our construction. I learned many things from Professor Nakayama's work on elliptic fibrations. His seminar talk in 1999 and 2000 was very helpful for completing this work. My original motivation was to understand his $\partial$-étale cohomology theory [N2] from another 'geometric' viewpoint (i.e. the logarithmic transformations). And in a private seminar, he listened to my talk very carefully and corrected errors. I had a chance to give a series of talks in a seminar at RIMS from April 2001 to July 2001. I am deeply grateful to the attendants: Professors Nakayama, Hiromichi Takagi and Osamu Fujino, who pointed out some errors. I would like to express sincere thanks to Professors Kenji Ueno and Goro Chuman for their constant encouragement.

\section{References}

[Be] Beauville, A., Variété Kähleriennes dont la premierer classe de Chern est null, J. Diff. Geometry, 18 (1983), 755-782.

[CS] Cho, K. and Sato, E., Smooth projective varieties dominated by smooth quadric hypersurfaces in any characteristic, Math. Zeit., 217 (1994), 553-565.

[De] Debarre, O., Images lisses d'une variete abelienne simple, C. R. Acad. Sci. Ser. I, 309 (1989), 119-122.

[F1] Fujiki, A., On automorphism groups of compact Kähler manifolds, Invent. Math., 44 (1978), 225-258.

[F2] - On a holomorphic fiber bundle with meromorphic structure, Publ. RIMS, Kyoto Univ., 19 (1983), 117-134.

[F3] - On the structure of compact complex manifolds in $\mathscr{C}$, in Algebraic Varieties and Analytic Varieties, Adv. Stud. Pure Math., 1 (1983), Kinokuniya and NorthHolland, 231-302. 
[Fm1] Fujimoto, Y., On rational elliptic surfaces with multiple fibers, Publ. RIMS, Kyoto Univ., 26 (1990), 1-10.

[Fm2] - Logarithmic transformations on elliptic fiber spaces II. Elliptic bundle case, Math. Ann., 288 (1990), 559-570.

[G1] Grassi, A., On minimal models of elliptic 3-folds, Math. Ann., 290 (1991), 287-301.

[G2] - Log contractions and equidimensional models of elliptic threefolds, J. Algebraic Geom., 4 (1995), 255-276.

[H] Horst, C., Compact varieties of surjective holomorphic endomorphisms, Math. Z., 190 (1985), 499-504.

[HL] Harbourne, B. and Lang, W., Multiple fibers on rational elliptic surfaces, Trans. Amer. Math. Soc., 307 (1988), No.1, 205-225.

[HM] Hwang, J. M. and Mok, N., Holomorphic maps from rational homogeneous spaces of Picard number 1 onto projective manifolds, Invent. Math., 136 (1999), 209-231.

[II] Iitaka, S., Algebraic Geometry, Springer-Verlag, GTM 76, 1977.

[Kaw1] Kawamata, Y., The crepant blowing-up of 3-dimensional canonical singularities and its application to degeneration of surfaces, Ann. of Math., 127 (1988), 93-163.

[Kaw2] - Abundance theorem for minimal 3-fold, Invent. Math., 108 (1992), 229-246.

[Kaw3] - Characterization of abelian Varieties, Comp. Math., 43 (1981), 253-270.

[Kob] Kobayashi, S., Hyperbolic Complex Spaces, Springer, Grund Math., 318, 1998.

[Kol1] Kollár, J., Flops, Nagoya Math. J., 113 (1989), 15-36.

[Kol2] - Shafarevich maps and plurigenera of algebraic varieties, Invent. Math., 113 (1993), 177-215.

[KoMiMo] Kollár, J., Miyaoka, Y. and Mori, S., Rationally Connected Varieties, J. Alg. Geom., 1 (1992), 429-448.

[KM] Kollar, J. and Mori, S., Birational Geometry of Algebraic Varieties, Cambridge Tracts in Math., 134, 1998.

[Mi1] Miyaoka, Y., On the Kodaira dimension of minimal 3-folds, Math. Ann., 281 (1988), 325-332.

[Mi2] - Abundance conjecture for 3-folds: case $\nu=1$, Comp. Math., 68 (1988), $325-332$

[MiMo] Miyaoka, Y. and Mori, S., A numerical criterion for uniruledness, Ann. of Math. (2), 124 (1986), 65-69.

[Mo1] Mori, S., Threefold whose canonical bundles are not numerically effective, Ann. of Math., 116 (1982), 133-176.

[Mo2] - Flip theorem and the existence of minimal models for 3-folds, J. Amer. Math. Soc., 1 (1988), 117-253.

[Mu] Mumford, D., Tata Lectures on Theta II, Birkhauser, Progr. Math., 43, 1984.

[N1] Nakayama, N., Local structure of an elliptic fibration, Preprint Tokyo Univ (1991), revised version, (1999) to appear.

[N2] - Global structure of an elliptic fibration, Preprint RIMS-1072 (1995), revised version, Preprint RIMS-1322 (2001).

[N3] - Projective 3-folds whose universal coverings are $\mathbf{C}^{3}$, the proceeding of symposium on vector bundles and algebraic geometry, Kyusyu University, January, 1997, organized by S. Mukai and E. Sato, 6-10.

[N4] phic to $\mathbf{C}^{n}$, J. Math. Soc. Japan., 51 (1999), No.3, 643-654.

[N5] - The lower semi-continuity of the plurigenera of complex varieties, Adv. Stud. Pure Math., 10 (1985), Algebraic Geometry, Sendai, 551-590.

[N6] - Ruled surfaces with nontrivial surjective endomorphisms, Preprint RIMS1286 (2000).

[PS] Paranjape, K. H. and Srinivas, V., Self maps of homogeneous spaces, Invent. Math., 98 (1989), 425-444.

[SF] Sato, E. and Fujimoto, Y., On smooth projective 3-folds with nontrivial surjective endomorphisms, Proc. Japan Acad. Ser. A Math. Sci., 74 (1988), No.10, 143-145. 
[U1] Ueno, K., On algebraic fiber spaces of abelian varieties, Math. Ann., 237 (1978), $1-22$.

[U2] - The appendix to Ashikaga, T., The degeneration behavior of the Kodaira dimension of algebraic manifolds, Tôhoku Math. J., 33 (1981), 193-214. 